\title{
Feasibility Study of Tuned-Resonator, Pulsating Cavitating Water Jet for Deep-Hole Drilling
}

Virgil E. Johnson, Jr, William T. Lindenmuth, Andrew F. Conn, Gary S. Frederick Hydronautics, Incorporated 7210 Pindell School Road Laurel, Maryland 20810

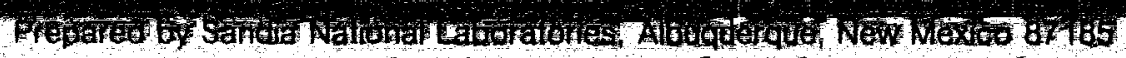
and Livermore, Calfornia 94550 for the United States Department of Energy under Contract DE-AC04-76DP00789

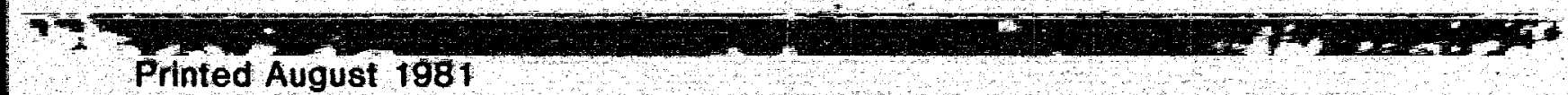

Printed August 1981 under Contract No. 13-5111 


\section{DISCLAIMER}

This report was prepared as an account of work sponsored by an agency of the United States Government. Neither the United States Government nor any agency Thereof, nor any of their employees, makes any warranty, express or implied, or assumes any legal liability or responsibility for the accuracy, completeness, or usefulness of any information, apparatus, product, or process disclosed, or represents that its use would not infringe privately owned rights. Reference herein to any specific commercial product, process, or service by trade name, trademark, manufacturer, or otherwise does not necessarily constitute or imply its endorsement, recommendation, or favoring by the United States Government or any agency thereof. The views and opinions of authors expressed herein do not necessarily state or reflect those of the United States Government or any agency thereof. 


\section{DISCLAIMER}

Portions of this document may be illegible in electronic image products. Images are produced from the best available original document. 
Issued by Sandia National Laboratories, operated for the United States Department of Energy by Sandia Corporation.

\section{NOTICE}

This report was prepared as an account of work sponsored by the United States Government. Neither the United States nor the Department of Energy, nor any of their employees, or any of their contractors, subcontractors, or their employees, makes any warranty, express or implied, or assumes any legal liability or responsibility for the accuracy, completeness or usefulness of any information, apparatus, product or process disclosed, or represents that its use would not infringe privately owned rights.

Printed in the United States of America

$$
\text { Available from: }
$$

Nationa1 Technical Information Service

U. S. Department of Commerce

5285 Port Royal Road

Springfield, VA 22161

Price: Printed Copy $\$ 6.00$, Microfiche $\$ 3.00$ 


\title{
SAND81- 7126 \\ UNLIMITED RELEASE \\ PRINTED AUGUST 1981 \\ UC- $-66 c$
}

FEASIBILITY STUDY OF TUNED-RESONATOR, PULSATING CAVITATING WATER JET FOR DEEP-HOLE DRILLING

Virgil E. Johnson, Jr. William $T$. Lindenmuth

Andrew F. Conn

Gary S. Frederick

HYDRONAUTICS, Incorporated

Laure1, Maryland

\begin{abstract}
This study presents the advantages of pulsing a submerged jet to increase its erosion capability (particularly as caused by cavitation) in augmenting deep-hole drill bits. Various methods of accomplishing the pulsation are presented and discussed. The most attractive systems uncovered are acoustic oscillators which passively accomplish pulsations in the flow at frequencies corresponding to a Strouhal number in the range of 0.2 to 1.0 . Such passive oscillators are assessed to be feasible candidates for development into practical deep hole drill bit systems and a long range plan for this research and development is presented and discussed.
\end{abstract}

*Work performed under Sandia National Laboratories Contract No. 13-5111 for the U.S. Department of Energy, Division of Geothermal Energy. 
FOREWORD

The study described in this report was conducted by HYDRONAUTICS, Incorporated, Laurel, Maryland. Portions of the experimental program were conducted under a subcontract to Professor Albert T. Ellis in his laboratory at the University of California at San Diego. The program was supported by the U.S. Department of Energy, Division of Geothermal Energy, under Sandia Laboratories Contract No. 13-5111.

The CAVIJET ${ }^{(}$cavitating fluid jet technology used in this program has been patented by HYDRONAUTICS, Incorporated* and additional patents related to tuned-resonator CAVIJET are being applied for. The CAVIJET technology is currently being developed for a variety of commercial applications.

Technical direction was provided by Sandia National Laboratories Division 4751, with $\mathrm{Mr}$. Dave Glowka as the Technical Project Officer.

* U.S. Patents Nos. $3,528,704 ; 3,713,699$ and $3,807,632$. Other U.S. and Foreign patents are pending or have been granted. CAVIJET is a registered trademark of HYDRONAUTICS, Incorporated. 


\section{ACKNOWLEDGMENTS}

The following organizations and individuals have made important contributions to the success of this phase of our investigations. The authors would like to express their sincere appreciation to:

- Sandia Laboratories - - Dr. Samue1 G. Varnado and Mr. David A. Glowka, for their support, advice, and encouragement throughout this program.

- NL/Hycalog - - Mr. Robert P. Radtke, whose inspiration, untiring enthusiasm, and guidance have kept this study on course since its inception.

- University of California, San Diego - - Professor Albert T. Ellis for his many creative efforts, as described in this report.

- HYDRONAUTICS, Incorporated - - Mr. Robert Bowen for his key role in the experimental work.

o Department of Energy, Division of Geothermal Energy - Mr. Clifton Carwile for providing the original encouragement and continued support of this program. 


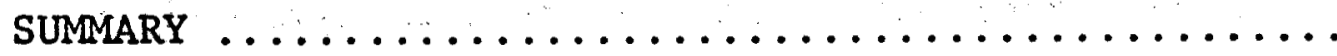

1

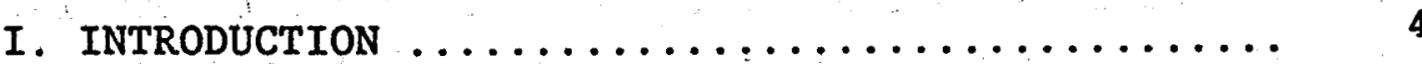

A. Background $\ldots \ldots \ldots \ldots \ldots \ldots \ldots \ldots \ldots \ldots$

B. Present Study .................... 10

II. REVIEW OF CURRENT JET PULSING TECHNOLOGY ...... 13

A. Expected Advantages of Pulsed Jets ........ 13

B. Advantages of Pulsing the Jet Near the Critical Strouhal Number .............. 17

C. Description and Critique of Pulsed Jet Concepts ........................ 19

1. General Concepts ................ 19

2. Concept Examples ................ 21

III. RECENT DEVELOPMENTS IN SELF-SUSTAINED FLUID OSCILLATORS

IV. DESCRIPTION OF PULSING CAVIJET ${ }^{\circledR}$ CONCEPTS SELECTED FOR FURTHER STUDY ............... 31

A. Organ-Pipe, Plain CAVIJET ${ }^{\circledR} \ldots \ldots \ldots \ldots \ldots \ldots$

B. Tandem-Orifice, Helmholtz Resonator ("Pulser") ........................ 32

C. Extended Nozzle-Orifice, Helmholtz Resonator ("Laid-Back-Pulser") .......... 35

D. Jet Driven Helmholtz Oscillator Supply to Plain CAVIJET ("Pulser-Fed" CAVIJET) ... 36

V. EXPERIMENTS $\ldots \ldots \ldots \ldots \ldots \ldots \ldots \ldots \ldots \ldots \ldots \ldots, 41$

A. Jet Cavitation From A Plain CAVIJET Nozzle With Forced Supply Excitation ...........

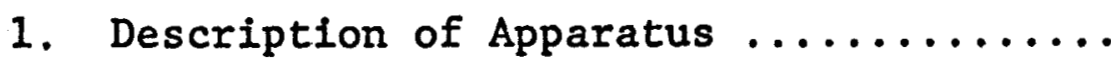

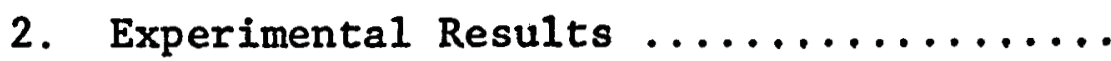


V. EXPERIMENTS (Continued)

B. Self-Excited Oscillator Experiments -

Air ........................ 46

1. Test Apparatus $\ldots \ldots \ldots \ldots \ldots \ldots \ldots \ldots .46$

2. Mean and RMS Velocities .......... 47

3. Experimental Results ............ 48

Organ Pipe CAVIJET .............. 48

"Pulser" ................... 49

"Laid-Back-Pulser" ................ 50

"Pulser-Fed" Orifice (Diffuser) ....... 50

"Pulser-Fed" Orifice (Sudden Expansion). 51

"Pulser" Frequency Coupling .......... 52

"Pulser" Modulation Amplitude ........ 54

C. Self-Excited Oscillator Experiments -

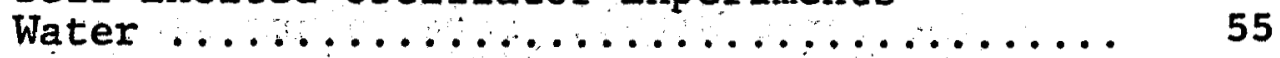

1. Test Apparatus .................. 55

2. Experimental Results ............. 55

"Pulser" Frequency Coupling ......... 55

Pressure Fluctuations ............ 56

Cavitation Inception . . . ......... 57

Cavitation Patterns .............. 58

Preliminary Erosion Tests ........... 59

VI. SUMMARY OF RESULTS AND ASSESSEMENT OF

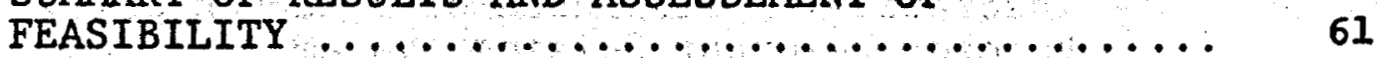

VII. LONG RANGE RESEARCH AND DEVELOPMENT PROGRAM .... 65

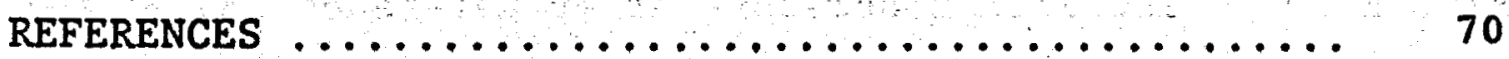




\section{LIST OF FIGURES}

$\underline{\text { Page }}$

FIGURE 1 - Comparison of Cavitating and Non-Cavitating Jets ............... 74

FIGURE 2 - Pulsing Concept Definitions........... 75 a. Square Wave Modulation

b. Sine Wave Modulation

FIGURE 3 - Comparison of Flow Patterns for Excited and Unexcited Submerged Jets ..... 76

FIGURE 4 - Schematic of the Jet Facility Used in Reference 22 Tests .............. 77

FIGURE 5 - Axial Profile of the Turbulence Intensity, Measured along the Centerline Under $2 \%$ Forcing at a Strouhal Number of 0.30 . The Curve Without Data Points Represents the Unforced Case (After Reference 22) ... 78

FIGURE 6 - Components of the Structural Modulation in a Jet Excited at $S=0.30$ (After

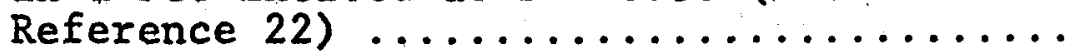

FIGURE 7 - Comparison of Shear Zone Cavitation in Submerged Structured and Unstructured Jets $\ldots \ldots \ldots \ldots \ldots \ldots \ldots \ldots \ldots \ldots \ldots . \ldots . \ldots$

FIGURE 8 - General Concepts for Pulsing Jets ....... 81

FIGURE 9 - Theoretical Performance of a Mechanically Forced, Resonant Pulsing System

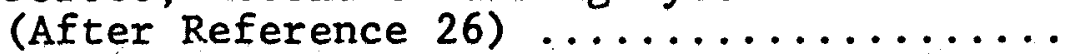

FIGURE 10 - Flow Rate Modulator (After Reference 28)...

FIGURE 11 - Fluid Amplifier Pulsing Device Suggested In Reference $29 \ldots \ldots \ldots \ldots \ldots$.

FIGURE 12 - Fülöp's Ultrasonic "Fluid Whistle" Oscillator Concept (After Reference 30) ...

FIGURE 13 - Examples of Geometries that Cause Self-Sustained Oscillation in Shear Flows (After Reference 34) 
$\underline{\text { Page }}$

FIGURE 14 - Flow Visualization of Vortex Impingement

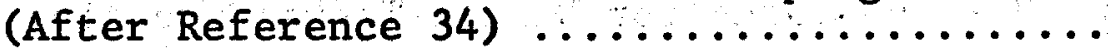

FIGURE 15 - Experimenta1 Set-Up for Jet-Driven Helmholtz Oscillators (After Reference 38).. 88

FIGURE 16 - Measured Peak RMS Chamber Pressure Fluctuations in Mode 2 (After Reference 38). 89

FIGURE 17 - Organ Pipe Resonant Cavity Concept ....... 90

FIGURE 18 - "Pulser" or Tandem-Orifice, Helmholtz Resonator Concept (With Organ Pipe Feed) .... . 91

FIGURE 19 - Conditions for Strong Jet Driven Helmholtz Oscillator (Pulser) Operation

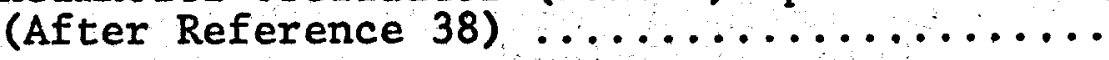

FIGURE 20 - Extended Jet Nozzle Casting and Insert for a Typical 9-7/8 Inch, 3-Cone Roller

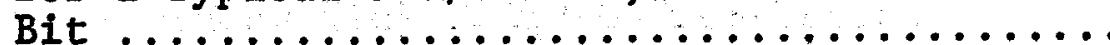

FIGURE 21 - "Laid-Back Pülser" Design Concept .......... 94

FIGURE 22 - "Pulser-Fed" CAVIJET Concept ........... 95

FIGURE 23 - Test Apparatus for Studying Pulsed Resonating Caviting Jets at U.C.S.D.

(A. T E11is) . . . . . . . . . . . . . . . . .

FIGURE 24 - Schematic of Laser Cavitation Detection

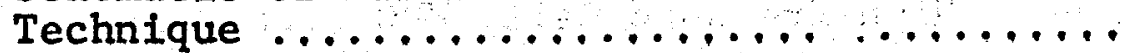

FIGURE 25 - Correlation Between Excitation and Laser Cavitation Detector for Resonant Operation..

FIGURE 26 - The Influence of Velocity and Excitation Frequency on the Formation of Discrete

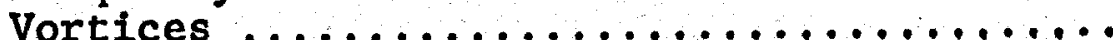

FIGURE 27 - Photographs of Excited and Unexcited Cavitating Jets $(6.3 \mathrm{~mm}$ Diameter CAVIJET) ... 100

FIGURE 28 - Jet Cavitation Inception for a Plaln CAVIJET Nozzle With and Without Excitation (at U.C.S.D.) 
Page

FIGURE 29 - Apparatus for Nozzle Tests With Air ..... 103

FIGURE 30 - View of Velocity Probe in the Exiting Jet Flow ................... 104

FIGURE 31 - Signal Processing Suite Schematic ........ 105

FIGURE 32 - View of Instrumentation ............ 106

FIGURE 33 - Centerline Velocity Distributions;

Organ Pipe Resonant Oscillator .......... 107

FIGURE 34 - Centerline Velocity Distributions; Typical "Pulser" ................... 108

FIGURE 35 - Centerline Velocity Distributions; "Laid Back Pulser" .................... 109

FIGURE 36 - Centerline Velocity Distribution; "Pulser-Fed" CAVIJET (Diffuser) ......... 110

FIGURE 37 - Center Velocity Distributions, "PulserFed" CAVIJET (Sudden Expansion) ......... 111

FIGURE 38 - Frequency Coupling and Modulation Amplitudes; LF Pulser In Air .......... 112

FIGURE 39 - Frequency Coupling and Modulation Amplitudes; $\mathrm{HF}$ Pulser in Air .......... 113

FIGURE 40 - High Pressure Cell, HPC ............ 114

FIGURE 41 - View of High Pressure Cell Showing Viewing Ports ..................... 115

FIGURE 42 - View of Piezoelectric Transducer In Wall of HPC .................... 116

FIGURE 43 - View of CAVIJET Atmospheric Pressure

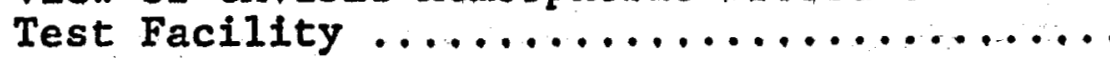

FIGURE 44 - Frequency Coupling; LF Pulser in

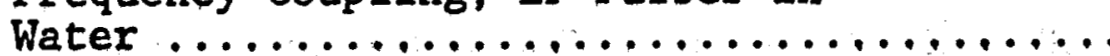


FIGURE 45 - Frequency Coupling; HF Pulser in Water .... 119

FIGURE 46 - Pressure Fluctuations in HPC, HF Pulser .... 120

FIGURE 47 - Effect of Cavitation Number on Pressure Fluctuation in

FIGURE 48 - Comparison of HF Pulser Modulations Measured in Air and Water ............. 121

FIGURE 49 - Cavitation Inception for Various

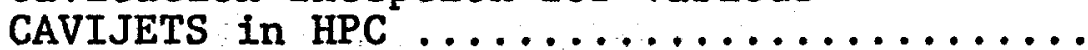

FIGURE 50 - Cavitation Pattern for Self-Excited

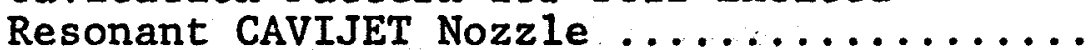

FIGURE 51 - Cavitation Pattern for CAVIJET

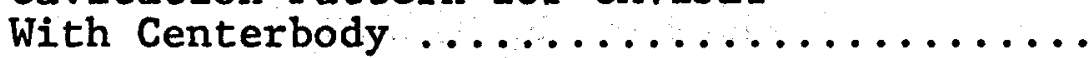

FIGURE 52 - Cavitation Pattern for Conventional Drill

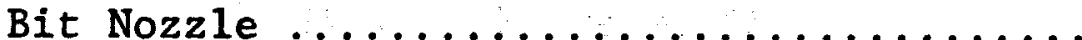

FIGURE 53 - Maximum Depth and Volume Erosion Histories From Tests in the CAVIJET Atmospheric

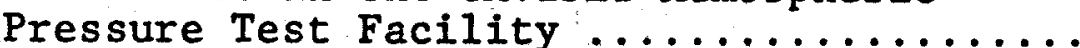

FIGURE 54 - Interaction of Various Activities in Overall R\&D Program 
NOTATION

A

$\mathrm{C}_{\mathrm{D}}$

D, d

F

$\mathrm{K}_{\ell}$

$\mathrm{K}_{\mathrm{N}}$

$L, \ell$

$\mathrm{M}$

N

$P$

$\operatorname{Re}$

$\mathrm{S}_{\mathbf{L}}$

$\mathbf{S}_{\mathbf{d}}$

$s_{j}$

U

$\forall$

$\mathrm{X}$

$c$

$e_{\dot{h}}$

f

$\dot{\mathrm{h}}$

n

$\mathrm{p}$

$\Delta \mathrm{p}$

q

t

$\Gamma$

$\lambda$

$\nu$

$\rho$

$\sigma$

Nozzle orifice area

$\mathrm{L}^{2}$

Nozzle discharge coefficient ND

Diameter

A function

Loss coefficient

ND

Mode coefficient

ND

Length

L

Mach number, U/c

ND

Mode number (integer $=0,1,2 \ldots$ ) -

Power

FL/T

Reynolds number, Ud/v

ND

Strouhal number, Helmholtz

chamber, $S_{L}=f \dot{L} / U$

ND

Strouhal number, nozzle

ND

diameter, $\mathrm{S}_{\mathrm{d}}=\mathrm{fd} / \mathrm{U}$

Strouhal number, jet diameter,

ND

$S_{j}=f d_{j} / U$

Jet velocity

$\mathrm{L} / \mathrm{T}$

Volume

$\mathrm{L}^{3}$

Distance from orifice

L

Speed of sound in fluid

$\mathrm{L} / \mathrm{T}$

Cutting rate effectiveness

$F^{-1}$

Frequency, $\mathrm{Hz}$

$\mathrm{T}^{-1}$

Rate of depth penetration

$\mathrm{L} / \mathrm{T}$

Exponent or integer

Pressure

$F / L^{2}$

Nozzle pressure drop

$F / L^{2}$

Dynamic pressure

$F / L^{2}$

Time

$T$

Circulation

$F^{2} / T$

Acoustic wavelength

L

Kinematic viscosity

$L^{2} / T$

Fluid density

$\mathrm{FT}^{2} / \mathrm{L}^{4}$

Cavitation number, $p_{a} / \Delta p$

ND 
Subscripts

1,2 and $\mathrm{e}$

L, d

S

$\mathbf{T}, \mathrm{c}$

Upstream, downstream and exit orifices

a

i

Length, diameter

Source pipe

Helmholtz chamber

Ambient

Incipient

j

Jet

p

Pipe

s

Steady

Superscripts

$x^{\prime}$
$\bar{x}$
$\star$

rms of fluctuating component

Temporal average or mean

Critical Strouhal number, for optimum jet structuring 


\section{SUMMARY}

A feasibility study has been completed, which had the objective of evaluating means for exciting resonant pulsations in cavitating water jets so as to produce ring vortex structures in the flow. In this manner, we anticipate increasing the degree of cavitation and, thereby, the jet's erosivity in order to enhance the performance of rotary mechanical bits used for deephole drilling. The study served to help answer several questions regarding the performance of systems that could achieve pulsations passively. The approach used to gain these answers coupled a review of relevant, current, jet pulsing technology with an ad hoc program of experiments and analyses. In view of the positive nature of our findings, the study culminated with the delineation of an R\&D program designed to achieve a practical passive pulsing jet-bit system.

The review of jet pulsing technology disclosed several schemes for creating low frequency $(\approx 100 \mathrm{~Hz})$ pulses that could apply to deep-hole drilling. These are not capable of the structuring that is sought for the high speed $(150-300 \mathrm{~m} / \mathrm{s})$ jets of interest. The desired high frequency pulsations $(\approx 10 \mathrm{kHz})$ can be generated passively, however, with Helmholtz oscillators and tuned (organ pipe) resonant chambers. One such device, an ultrasonic "whistle", was patented in 1978 (Reference 30 ); however, this device is not capable of structuring the jet for enhanced cavitation erosion.

Experiments conducted under subcontract by Dr. Albert Ellis at UCSD show that jets do become structured, with enhanced cavitation in ring vortices, when low level forced excitation (two percent of the jet dynamic pressure) is provided at critical frequencies. These frequencies correspond to Strouhal numbers (based on the jet diameter) near 0.34 and 0.68 . 
Experiments with jets in air confirmed the earlier results of tests reported by Morel that showed structured velocity modulations having rms intensities as large as 60 percent (of the mean jet velocity) could be passively induced with Helmholtz type oscillators or "Pulsers." Similarly, we found simple organ-pipe chambers capable of achieving somewhat lower intensities. Strong oscillations can be achieved with simple "Pulsers" at jet Mach numbers up to 0.12 (jet velocities up to $180 \mathrm{~m} / \mathrm{s}$ ) so long as internal cavitation does not interfere with the resonance mechanism (in water). We also found that the useful Mach number range could be increased when a "Pulser" is placed upstream, feeding a plain CAVIJET ${ }^{\circledR}$ nozzle. This concept has the advantage of no cavitation within the "Pulser" section, but suffers from associated energy losses.

A simplified analysis of the effect that pulsing could have on jet erosivity indicated that the jet's performance may be expected to double when the amplitude of the nozzle pressure drop fluctuations is about 90 percent of the mean pressure drop, as compared to a steady jet having an equivalent mean pressure drop.

The extensive work that was uncovered in the review of jet pulsing techniques permitted an expansion of the planned experimental program to include tests in water. These experiments in water showed that self-excited pulsations did cause more intense cavitation and nearly doubled the cavitation number for incipient jet cavitation. The simple "Pulser" configuration did not give rise to the degree of jet structuring that was observed with active excitation of a plain CAVIJET, however.

While time did not permit extensive rock erosion tests to be performed, brief experiments in water at very low cavitation number showed a 20 percent increase in volume erosion rate for a "Pulser" nozzle compared to a plain, steady CAVIJET. 
The simple concepts that were uncovered for passively pulsed jets can be designed to replace conventional nozzles in all types of drill bits. It is very probable that improved drilling performance will be achieved; however, the magnitude of the improvement cannot be ascertained without further development and trials.

An R\&D program is prescribed for creating a complete practical system for passively pulsed jets in bits. This program consists of several interactive elements:

- "Pulser" internal erosion studies,

- "Pulser" design procedure,

- Flow visualization studies.

- Nozzle performance optimization - air,

- Nozzle performance optimization - water,

- Laboratory "bit" tests,

- Field trials. 


\section{INTRODUCTION}

A. Background

The Department of Energy Publication "Blueprint For a Comprehensive Federal Drilling Technology Development Program,"

"February 1978 (Reference 1), gives high priority to developing an operational jet erosion drilling system with a goal for commercialization in the 1985 time frame. The background which justifies placing such emphasis on utilizing the erosion associated with high pressure fluid jets incorporated in various types of bits is described in the following paragraphs.

Historically, improved control of the fluid dynamics of the drilling mud has consistently contributed to increased performance of oil well drill bits. The perfect cleaning theory proposed by Maurer (2) for roller bits emphasized the importance of hydraulic chip removal for maximizing the drilling rate. The performance of diamond drill bits also depends on suitable fluid flow to clean the bit face efficiently.

Nozzles were first tested in roller bits in 1949. By 1955 the use of nozzles to direct the flow of the drilling mud was a standard feature in roller bit design. As a result, there was a marked increase in roller bit penetration rates. In recent years, extended nozzles in roller bits have created more efficient use of available hydraulic horsepower. Drilling rates in weak formations have been increased by up to 25 percent over the rates for similar bits without nozzles.

Diamond drill bits with jet nozzles have been tested experimentally since the mid 1950s. The advent of the General Electric Stratapax drill blank has led to the development of new bit designs which incorporate nozzles to clean cuttings from the bit 
face. More efficient use of hydraulic energy at the bit face has consistently resulted in major improvements in drill bit performance (3).

The use of fluid jets to augment the cutting action of various mechanical devices for drilling, tunneling, and mining applications is now being actively studied by investigators throughout the world $(4,5,6,7)$. The reason for this wide interest in the exploitation of jets is the inherent power delivery advantage over a purely mechanical device. As described by Maurer (3) and Bobo (8), because of material strength limitations, a typical rotary drill (20 cm dia.) can deliver only about 19 to $75 \mathrm{~kW}$ (25 to $100 \mathrm{hp}$ ) to the rock. The power deliverable via a fluid jet is governed only by practical questions of system costs and the pressure limits on key components for any particular application. Thus, water jets up to $414 \mathrm{MPa}(60,000 \mathrm{psi})$ are now being operated in many commercial installations $(4,5,6,7)$.

Drilling tests with fluid (water, drilling mud) jets, either alone or in conjunction with roller or diamond cutting surfaces $(9,10,11)$ have proven that, at sufficiently high pump pressures, dramatic increases in drilling rates can be achieved. For instance, Maurer (9) and Pols (10), each using high-pressure conventional fluid jets to assist the action of mechanical bits, were able to drill two to three times faster than the rates achieved by ordinary bits in the same formations. Pressures in these tests have, however, typically been in the range of $69 \mathrm{MPa}$ to $103 \mathrm{MPa}(10,000$ to $15,000 \mathrm{psi})$. Thus, the pressures which have been used in these conventional jet drilling studies are well above the currently available mud pump pressures on rigs now in operation. These are typically 14 to $21 \mathrm{MPa}(2,000$ to $3,000 \mathrm{psi})$, with a few off-shore rigs using up to $31 \mathrm{MPa}(4,500$ psi). Reliable and safe pumps and supporting components could 
readily be introduced to provide pressures up to $52 \mathrm{MPa}(7,500$ psi) at many rigs, if sufficient increases in the rate of penetration could be demonstrated.

In a preliminary feasibility study (12) the possiblity of using CAVIJET ${ }^{\circledR}$ cavitating fluid jet nozzles for rock cutting under elevated ambient conditions was examined. These tescs, with water as the working fluid, demonstrated that the erosive action of a $6.4 \mathrm{~mm}(0.25 \mathrm{in.})$ CAVIJET nozzle continued to be observed up to ambient pressure of $20.7 \mathrm{MPa}(3,000 \mathrm{psi})$. In comparative tests with a "Leach \& Walker" nozzle (13), the CAVIJET@ nozzle showed a higher volume removal efficiency under elevated ambient pressures. Specimens of Berea sandstone and Indiana limestone were used, with pressure drops across the nozzles ranging from 6.9 to $17.2 \mathrm{MPa}(1,000$ to $2,500 \mathrm{psi})$. The success of this preliminary study provided the basis for the first phase of a new program, which is described in Reference 14.

The CAVIJET cavitating fluid jet technology (Reference 14), is being successfully developed for a variety of commercial cleaning and cutting applications. These include removal of marine fouling $(15,16)$, cutting coal $(17,18)$, cleaning explosives and propellants from munitions $(19,20)$, and rock cutting $(21,23)$. However, in all of these applications the working fluid was water and the ambient pressure surrounding the jet was essentially atmospheric.

The study of the feasibility of using the CAVIJET cavitating jet technology to augment the drilling action of deep-hole geothermal drill bits, so as to increase the rates of penetration, described in Reference 14, was initiated to answer the following questions : 
a. Will there be sufficient erosion by cavitation from CAVIJET nozzles, when operated under the elevated ambient pressures down-hole, to increase mechanical drill bit penetration rates?

b. What is the effect of substituting drilling mud for the ordinary water which has been the working fluid for all previous CAVIJET cavitating jet operations?

c. How can the CAVIJET nozzles be introduced into drill bits, so as to effectively augment the mechanical cutting action, and also satisfy the requirements for bit cooling and chip - removal?

Some of the specific conclusions of the Reference 14 study which served to answer these feasibility questions were:

- Effect of nozzle design: Single nozzle tests confirmed that submerged jets are more erosive when they cavitate and that jets from CAVIJET nozzles designed to increase the degree of cavitation are more erosive than jets issuing from conventional nozzles.

- Effect of drilling mud: Single nozzle tests revealed that no adverse influence on CAVIJET nozzle rock cutting was seen when muds having densities up to $1.4 \mathrm{gm} / \mathrm{cm}^{3}$ (12 $\mathrm{lb} / \mathrm{gal}$ ) were substituted for water; in fact the use of mud increased the erosivity of the jet cutting.

- Effect of nozzle pressure drop $(\Delta p)$ : Single nozzle tests revealed that the cutting rates of cavitating jets vary approximately as the cube of the nozzle pressure drop over the entire range of cavitation numbers tested.

- Effect of ambient pressure $\left(p_{a}\right)$ : The effect of ambient pressure is characterized for all values of $\Delta p$ by the 
cavitation number, $\sigma=\mathrm{p}_{\mathrm{a}} / \Delta \mathrm{p}$. Single nozzle tests revealed that the cutting rate for all nozzles tested increased substantially from noncavitating conditions ( $\sigma * 1$ to 2 ) to a maximum occurring at approximately $\sigma=0.4$, and then decreasing with further reduction in the cavitation number. Thus, for pump pressures of 13.8 $\mathrm{MPa}$ (2000 psi), cavitation will substantially increase the cutting rate of the nozzles tested at hole depths less than about $1220 \mathrm{~m}$ ( $4000 \mathrm{ft})$. This maximum depth is directly proportional to the pressure drop (2440 m $(8000 \mathrm{ft})$ for $\Delta \mathrm{p}=27.6 \mathrm{MPa}(4000 \mathrm{psi}))$ and can be further increased for a given pressure drop by improving the nozzle design so as to further stimulate cavitation.

- Effect of nozzle design on roller bit cutting rates: Tests were conducted on two roller bits under simulated downhole conditions with conventional nozzles and with CAVIJET nozzles. Since these tests were conducted using nozzles of the same diameter rather than nozzles of equivalent discharge a direct comparison is not possible. However, the results indicate that if CAVIJET nozzles are sized to give equivalent discharges when substituted for conventional nozzles, a modest improvement in drilling rate should result. If future studies evolve nozzle designs which further enhance cavitation then these nozzles should further increase the drilling rate when substituted for conventional nozzles.

One of the specific recommendations of Reference 14 was to seek ways of increasing the point of cavitation inception and thus the intensity of erosion of the jets.

The reason for emphasizing cavitating jets in a program directed at achieving improved drilling rates through the 
utilization of high pressure erosion jets is that cavitation will occur in the upper regions of the hole being drilled and when the jet cavitates it is more erosive. Thus it is desirable to extend the range of depth over which cavitation will occur and to increase the degree or amount of cavitation as much as possible.

In Figure 1 the important dimensionless parameters, cavitation number, $\sigma$, and incipient cavitation number, $\sigma_{1}$, are defined. The incipient cavitation number, $\sigma_{ \pm}$, is the ratio of the ambient. pressure surrounding the jet to the pressure drop across the nozzle at which cavitation is first observed. It then follows that operating cavitation numbers greater than $\sigma_{1}$ will be cavitation free and operating cavitation numbers less than $\sigma_{1}$ will cavitate. Furthermore the amount of cavitation increases as the value $\left(\sigma_{i}-\sigma\right)$ increases.

As discussed in Reference 14, for a given value of the Reynolds number $(U d / v$ ) (where $U=$ jet velocity, $d=$ jet diameter and $v=$ kinematic viscosity), the value of $\sigma_{1}$ is believed to be unique for a given nozzle shape operating under steady flow conditions. If the jet flow is oscillated (either passively or actively) then the value of $\sigma_{1}$ may also be frequency dependent.

As discussed in Reference 14, and illustrated in Figure 1, it is well established that cavitating jets are more erosive than non-cavitating jets because the cavities which collapse on or near the material to be eroded generate pressures orders of magnitude greater than the dynamic pressure of the jet. It therefore follows that any high pressure jet system will cavitate during deep hole drilling down to the depth where $\mathrm{p}_{a} / \Delta \mathrm{p}=\sigma_{i}$. In order to maximize the range of depths over which cavitation occurs (and the degree of cavitation that occurs at a given depth) it 
is therefore of interest to maximize the value of $\sigma_{1}$ (as well as focus the cavity collapses in desirable ways). The patented CAVIJET concepts described in Reference 14 accomplish this by properly shaping the nozzle and incorporating internal devices.

The motivation for the work described in the present report is based on the postulate that if the jet is somehow excited (passively or actively) so as to cause a pulsating flow then optimum conditions will be found for which $\sigma_{1}$ is greater than the value for steady flow. Furthermore it is expected that the resulting erosion will be enhanced not only because of the increase in $\sigma_{1}$ but also because of the higher stagnation pressures (during the oscillation peaks) available to collapse cavities.

B. Present Study

The present study was carried out to investigate the feasibility of increasing the point of cavitation inception and the intensity of cavitation erosion as depicted in Figure 1, by causing the CAVIJET ${ }^{8}$ flow to pulsate or oscillate. As discussed in the body of this report, it is obvious that an oscillating flow having equivalent energy to a steady flow will tend to cavitate significantly more during the crest of the oscillation than the equivalent steady flow. Furthermore, the finding of Reference 14 that the intensity of erosion is nominally proportional to the cube of the pressure drop across the nozzle leads to the conclusion that an oscillating flow should have greater net erosion capability or intensity because the gain in intensity during the crest of the oscillation will be much greater than the loss during the trough of the oscillation. These observations should be true regardless of the details of the-particular-CAVIJET nozzle used. Thus, improved steady flow CAVIJET nozzle designs may be made even more effective by incorporating the concept of pulsing the flow. 
Although the desired flow oscillation can clearly be achieved in a variety of ways, the present study gave primary attention to the observation made in Reference 14 that the cavitation observed in the shear zone of a cavitating plain CAVIJET tended to be moderately structured corresponding to ring vortices having a preferred period. Furthermore, the work in air jets described in Reference 22 has shown that a jet, excited with only moderate energy at a preferred frequency corresponding to a Strouhal number, $S_{d}=f d / U$, of approximately 0.3 , becomes highly structured into a sequence of ring vortices persisting for several jet diameters downstream of the nozzle exit. At the preferred frequency, it was noted in Reference 22 that the velocity fluctuations in the jet were nearly an order of magnitude greater than the input fluctuations.

The principal direction of the present study was to examine ways of taking advantage of this remarkable jet structuring phenomenon so as to achieve large oscillations in cavitating jet flows. Particular attention was paid to the possibility of further amplifying the magnitude of the oscillation through the use of resonant systems, especially those that might be self excited.

The objective of the present study was to answer the following questions, or at least contribute to their answer:

- If a high pressure liquid jet is excited at its source, will there be preferred frequencies at which the jet structures into ring vortices and does the jet cavitate at higher cavitation numbers?

- Are there existing systems or systems under development which might be adapted in deep-hole drilling to achieve a passive oscillation in the jet flow at frequencies near the frequency required to cause discrete ring vortices to form? 
- Are there new concepts or modifications to existing concepts of passive systems capable of providing the jet excitation required to structure the jet and cause large pulsations in the jet flow? If so, do existing experiments or new simple "bench-type" experiments at least show the concept to have promise?

- If a passive oscillator system for improving the performance of high pressure jet augmented drill bits is uncovered that shows promise, what is the possibility that such a system can, through further research, be developed into a practical or feasible system?

- If it is judged that a passive oscillator system for improving the performance of jet augmented bits warrants further development, what is the research and development program required to achieve a practical system?

This report presents the results of the study carried out in search of answers to these questions. 


\section{REVIEW OF CURRENT JET PULSING TECHNOLOGY}

\section{A. Expected Advantages of Pulsed Jets}

A principal conclusion reached in Reference 14 is that the rate of penetration, $h$, of a cavitating water $j$ et increases exponentially with an increase in the pressure drop across the nozzle, $(\Delta \mathrm{p})$. That is,

$$
\dot{\mathrm{h}} \propto(\Delta \mathrm{p})^{\mathrm{n}}
$$

For cavitating jets, Reference 14 reports that the exponent $\mathfrak{n}$ is approximately 3 for the flow conditions and rock types tested. In Reference 23, the exponent has been found to be as high as 6.9, particularly for the harder types of rock such as granite.

Even non-cavitating high pressure jet cutting tests conducted in air with conventional jets reveal penetration rate variation with $(\Delta p)$ such that $n \geqslant 1$ in Equation [1]; examples may be found in Refs, 4-7, 24 and 25. When the stand-off distance in such tests is small so that the erosion is caused principally by the application of the stagnation pressure to the rock face, the exponent is, as expected, generally approximately one. However, if the stand-off distance is large enough for the jet to begin to break up, the exponent, $n$, in the Equation [1] is generally greater than 1 .

It is unfortunate that Equation [1] has been used so frequently in the literature to correlate the influence of nozzle pressure drop with the penetration rate because it gives the impression that it is known that the fundamental relationships between these variables must have the form of Equation [1]. 
Only in the simplest case of a non-cavitating steady jet operating at short stand-off distances (where the impact pressure is $\Delta \mathrm{p}$ and thus, $\mathrm{n} \approx 1$ ) does Equation [1] correctly contain the expected relationship between $\Delta \mathrm{p}$ and the actual impact pressure. Reference 21, for example, points out that for cavitation erosion the cavity collapse pressure should theoretically vary like $\Delta \mathrm{p} \cdot \exp (\Delta \mathrm{p} \cdot$ constant $)$. However, Equation $[1]$ has been found most useful to date in correlating cavitation erosion data. The fact that the exponent $n$ varies so widely is evidence that the fundamental relationships are not known or at least are not being used.

Regardless of the foregoing discussion, related to the adequacy of Equation [1], there is no doubt that in most cases of jet cutting and in all cases in which the jet is cavitating, Equation [1] correctly indicates very large increases in penetration rate with increases in the nozzie pressure drop, i.e.,increasing jet velocity.

The advantages of pulsing a cavitating jet whose penetration rate varies with nozzle pressure drop according to Equation [1] are apparent if the flow is pulsed in a square wave such as shown in Figure 2a. The flow is assumed to be off during one half cycle and on at a value equal to $2 \Delta \mathrm{p}_{\mathrm{s}}$, during the remaining half cycle. The mean value, $\Delta \mathrm{p}_{s}$, is used for comparison with another jet flow operated over the entire cycle at a steady nozzle pressure. Assuming nozzles with equal area, $A$, having equal discharge coefficients, $C_{D}$, the following ratios of power $(P)$ and drilling rate (h) will result when Equation [I] is used.

$$
\frac{P_{\text {pulsed }}}{P_{\text {steady }}}=\frac{\frac{1}{2} C_{D} A\left(2 \Delta p_{s}\right)^{3 / 2}}{C_{D} A \Delta p_{s}^{3 / 2}}=\sqrt{2}
$$




$$
\frac{\dot{h}_{\text {pulsed }}}{\hat{h}_{\text {steady }}}=\frac{\frac{1}{2}\left(2 \Delta p_{s}\right)^{n}}{\left(\Delta p_{s}\right)^{n}}=2^{n-1}
$$

Defining cutting rate effectiveness, $e_{h}$, as $h / P$,

$$
\frac{e_{\dot{h}, \text { pulsed }}}{e_{\dot{h}, \text { steady }}}=2^{\mathrm{n}-3 / 2}
$$

Thus, if $\mathfrak{n} \geqslant 1.5$ in Equation [1], there is a clear ideal advantage to pulsing the jet. Since the value of $n$ for cavitating jets has experimentally been found to be 3 or greater, the ideal advantage given by Equation [4] becomes so large that an overall advantage should be expected even when the additional real losses associated with achieving such a pulsing system are included.

The ideal relationships given by Equations [2-4] for a square wave may also be developed for a sinusoidal pulsing or oscillating flow. Referring to Figure $2 b$ where the unsteady pressure drop $\left(\Delta \mathrm{p}_{\text {osc }}\right)$ is given by the equation

$$
\Delta \mathrm{p}_{\mathrm{osc}}=\overline{\Delta \mathrm{p}}+\sqrt{2} \mathrm{p}^{\prime} \sin \omega \mathrm{t}
$$

where $\mathrm{p}^{\prime}$ is the rms fluctuation about $\Delta \overline{\mathrm{p}}$.

$$
\frac{P_{\text {osc }}}{P_{\text {steady }}}=\frac{C_{D} A \frac{1}{2 \pi} \int_{0}^{2 \pi}\left(\overline{\Delta p}+\sqrt{2} p^{\prime} \sin x\right)^{3 / 2} d x}{C_{D} A\left(\Delta p_{s}\right)^{3 / 2}}
$$

$$
\frac{\dot{h}_{\text {osc }}}{\bar{h}_{\text {steady }}}=\frac{\frac{1}{2 \pi} \int_{0}^{2 \pi}\left(\Delta \bar{p}+\sqrt{2} p^{\prime} \sin x\right)^{n} d x}{\left(\Delta p_{s}\right)^{n}}
$$




$$
\frac{e_{h_{, \text {osc }}}}{\mathrm{e}_{\mathrm{h}, \text { steady }}}=\left(\frac{\Delta \overline{\mathrm{p}}}{\Delta \mathrm{p}_{\mathrm{s}}}\right)^{\mathrm{n}-3 / 2} \cdot \frac{\int_{0}^{2 \pi}\left(1+\frac{\sqrt{2} \mathrm{p}^{\prime}}{\Delta \overline{\mathrm{p}}} \sin \mathrm{x}\right)^{\mathrm{n}} \mathrm{dx}}{\int_{0}^{2 \pi}\left(1+\frac{\sqrt{2} \mathrm{p}^{\prime}}{\Delta \overline{\mathrm{p}}} \sin \mathrm{x}\right)^{3 / 2} \mathrm{dx}}
$$

For the case of $n=3$ Equation [8] results in the following approximate equation, for $\sqrt{2} \mathrm{p}^{\prime} / \Delta \mathrm{p}<<1$ :

$$
\frac{\mathrm{e}_{\dot{\mathrm{h}}, \text { osc }}}{\mathrm{e}_{\mathrm{h}, \text { steady }}} \approx\left[\frac{1+3\left(\frac{\mathrm{p}^{\prime}}{\left.\frac{\Delta \overline{\mathrm{p}}}{}\right)^{2}}\right.}{1+3 / 8} \frac{\left(\frac{\mathrm{p}}{\Delta \overline{\mathrm{p}}}\right)^{2}}{\left.\frac{\Delta \overline{\mathrm{p}}}{\Delta \mathrm{p}_{\mathrm{s}}}\right)^{3 / 2}}\right.
$$

If $\Delta \overrightarrow{\mathrm{p}}=\Delta \mathrm{p}_{\mathrm{s}}$, and $\mathrm{p}^{\prime}=\Delta \overline{\mathrm{p}} / \sqrt{2}$ (i.e., amplitude equal to mean pressure) in Equation [9], the ideal specific penetration rate advantage (for this case with $n=3$ ) is 2.1 compared to 2.83 for the square wave shown in Figure $2 a$. If the amplitudes of these modulations were halved, the ideal advantages decrease to 1.31 and 1.59 , respectively.

The foregoing simple analysis is presented as evidence of the advantages of pulsing high pressure jets and provides a gross estimate of the magnitude of this advantage. The details of the actual complex jet erosion process for a pulsing submerged jet are essentially unknown.

For example, the analysis completely neglects that pulsing the jet causes greater cavitation in the high pressure drop portion of the cycle and less in the low portion of the cycle. Furthermore, the analysis is quasi-steady and assumes the flow pattern to be independent of frequency. Actually, it has recently 
been discovered that the flow pattern in a submerged jet is dramatically altered when it is pulsed at its source at a critical frequency and to accomplish this structuring the level of $p^{\prime} / \Delta \bar{p}$ need be only a few percent. As pointed out in the following section, this restructuring of the jet flow by pulsing at the proper frequency must result in changes to the jet erosion intensity in a manner completely different from the simple concept of the $(\Delta p)^{3}$ law. However, it is expected that the erosion intensity continues to be strongly dependent on $\Delta p$ as described by such an exponential law.

B. Advantages of Pulsing The Jet Near The Critical Strouhal Number.

Crow and Champagne in Reference 22 report that an air jet from a nozzle can structure the shear zone eddies in the first few diameters into discrete ring vortices. This will occur if the inlet flow is pulsed at a frequency near a critical strouhal number equal to 0.3 or its multiples, where the strouhal number is defined as $\mathrm{fd} / \mathrm{U}_{e}$.

Figure 3 illustrates the idealized difference in flow patterns between the traditional one for unexcited submerged jets and the structured flow pattern that results for a jet excited at the critical frequency. The complex interaction between the discrete ring vortices which results in their break up is currently a subject of much interest in fluid mechanics, However most experiments to date show that break up does not occur before several jet diameters is reached. In practical deep-hole drilling applications, it is the first few diameters of the jet length that are of most interest.

Figure 4, reproduced from Reference 22 , shows a schematic of the test apparatus used in the experiments. The loudspeaker denoted as $L$ provided the input excitation for the jet exhausting at $N$. 
Figure 5, reproduced from Reference 22, shows the magnitude of jet velocity oscillation (or turbulence intensity), $u / U_{e}$ for the excited and unexcited cases. In the nomenclature of Reference 22, $\mathrm{u}$ denotes the (RMS) difference between the average exit velocity $\mathrm{U}_{\mathrm{e}}$ and the absolute velocity. Figure 6 shows that the energy in the jet oscillations which occur are almost entirely in the frequencies corresponding to either $S_{d}{ }^{*}=0.3$ or the harmonic 0.6 .

The important point to note in Figure 5 is that an input excitation value of $u / U_{e}=0.02$ results in a value of $u / U_{e}$ at an axial distance of four diameters of approximately 0.18 . The conclusion reached in Reference 22 is that the unstable vortex sheet surrounding the jet preferably amplifies the wave length corresponding to the excitation frequency. If $\mathrm{s}_{\mathrm{d}}{ }^{*} \approx 0.3$ or its $2^{\text {nd }}$ harmonic 0.6 , then the vortex sheet rolls into discrete ring vortices which convect along the jet periphery at speeds less than the jet speed (approximately two thirds of the jet speed).

One postulate of the present study was that a structured jet (i.e., with discrete $r$ ing vortices having an approximate spacing of $\mathrm{d}$, the jet diameter) should cavitate at a higher ambient pressure and/or more violently under the same pressures than an unstructured jet (i.e., with eddies one or two orders of magnitude smaller than d). As illustrated in Figure 7 this postulate was based on the fact that the circulation $\left(r_{2}=U \lambda_{2}\right)$ for the structured ring vortices will be one or two orders of magnitude greater than the circulation $\left(\Gamma_{1}=U \lambda_{1}\right)$ associated with the fine scale vortices of the traditional unstructured jet. The minimum pressure in the flow field is at the center of these eddies in the shear zone and the incipient cavitation number will be proportional to $\Gamma / U^{2}$ or $\lambda^{2}$ as shown in Figure 7 . Thus a jet whose velocity is oscillated at a Strouhal number of 0.3 should not only be more erosive because of the pulsing effect discussed in the previous section, but also because the degree of cavitation should be increased at a given operating cavitation number. That is $\sigma_{i, 2}>\sigma_{1,1}$. 
For deep-hole drilling, nominal jet velocities of 100 to $300 \mathrm{~m} / \mathrm{s}$ ( 300 to $1000 \mathrm{fps}$ ) and nozzle diameters of 6 to $12 \mathrm{~mm}$ are typically under consideration. If such nozzle flows are to be oscillated at Strouhal numbers of 0.3 to 0.6 , the frequency range of practical interest is 2.5 to $30 \mathrm{kHz}$. As discussed in the following section; such high frequencies tend to rule out mechanical or active pulsing mechanisms including passive systems utilizing fluid amplifiers which are currently in use for low frequency jet pulsing.

At the outset of this study, the high frequencies involved, corresponding to sound wave lengths in water of less than $0.3 \mathrm{~m}$ ( $1 \mathrm{ft.}$ ), suggested that acoustic oscillator or resonator concepts should be of particular interest and the principal goal of the study was to examine the feasibility of such concepts.

The following sections describe and critique the types of jet pulsing concepts considered in the study and examples of those concepts that were uncovered in the initial literature survey.

C. Description and Critique of Pulsed Jet Concepts

1. General Concepts. Figure 8 illustrates the categories of pulsing techniques that were conceived at the outset of the present study. A literature survey was then conducted to search for examples of the state of the art in each category and to add to the list if other types were uncovered.

Figure 8a illustrates the most straightforward type of mechanical pulsing, that is, piston displacement. A piston is oscillated upstream of the jet orifice in a chamber such that the impedance in the direction of the main flow source is high and in the direction of the jet nozzle the impedance is low. An obvious amplification of the pressure oscillation at the nozzle can be achieved by establishing a standing wave resonance in the system as discussed in a subsequent section. 
Figure $8 \mathrm{~b}$ illustrates another mechanical pulsing concept involving oscillatory throttling of the flow supply to the nozzle. Proper sizing of the supply geometry could again be used to set up resonance and thus amplify the magnitude of the oscillation of the jet flow.

Figure $8 \mathrm{c}$ illustrates another type of valve oscillator which does not require moving parts. The system utilizes fluid amplifier techniques such as the one illustrated to accomplish the oscillation. This device oscillates the flow back and forth about the splitter plate as follows: flow on one side causes a positive pressure to be fed back through the return path ( $B$ ' to $A^{\prime}$ or $B$ to $A$ ); this positive pressure applied at the jet root forces the jet to the alternate path which then sends back a positive signal to force the jet back again to repeat the process. This type of oscillator is ideal for dividing and oscillating the flow between two nozzles and thus achieving the on-off type of oscillation shown in Figure 2 . However, it seems difficult if not impossible to operate such a device at the high power levels required in deep-hole drilling at the high frequencies desired in the present study.

Figure 8d illustrates the simplest possible acoustic oscillator pulsing device: an organ-pipe supply chamber. If the supply line is contracted at a distance $L$ upstream of the final jet nozzle contraction, a standing wave whose length is approximately $2 \mathrm{~L}$ will exist in this chamber. The wave amplitude is dependent on the energy content of flow oscillations corresponding to a frequency equal to $c / 2 \mathrm{~L}$, where $c$ is the speed of sound in the liquid. If the organ-pipe length is tuned to a frequency which is amplified by the jet, the oscillation should grow in amplitude and cause a strong jet pulsation. The actual magnitude of amplification is best determined experimentally. This simple self-excited acoustic oscillator seems well suited to taking advantage of the preferred 
jet structuring frequency discussed in earlier sections of this report.

Figure $8 \mathrm{e}$ illustrates another version of the organ-pipe acoustic resonator in which the organ-pipe is replaced by the Helmholtz resonator. At the beginning of the present study, this device looked very promising if the jet would pass through the Helmholtz oscillator without excessive energy loss. As pointed out in a following section, it was discovered that extensive experiments had recently been carried out in air on such an oscillator and the results of these experiments showed that very large jet pulsations could be achieved with practically no energy loss. Furthermore, as discussed in a following paragraph, an acoustic oscillator or "underwater whistle" similar to the concept illustrated in Figure 8e has been proposed (Reference 30) for deephole drilling. However, according to the patent description, the device described would not be suitable for structuring the jet for enhanced cavitation.

A literature survey was made to establish "the state of the art" as applied to achieving fluctuations in the speed of high pressure jets which might be applied to deep-hole drilling. Specific examples of the concepts shown in Figure 8 were sought as well as other methods that might exist. The pertinent findings of this survey are summarized in the following paragraphs.

2. Concept Examples.

Maurer: Maurer in Reference 24 presents an extensive review prepared in 1979 of both continuous and pulsed high speed jet drilling concepts. All except one of the pulsed concepts described involve methods of achieving single high pressure pulses at a very low rate of repetition and thus are not of interest for the present study. Only the continuous modulated jet developed by Nebecker is of interest because of the high frequencies that are claimed for the device. This device is of the type shown in Figure $8 \mathrm{~b}$ and is described in more detail later. 
Wylie: In Reference 26, Wylie's description of the advantages of pulsing jets is similar to the discussion given in a previous section of this report. Furthermore Wylie points out the important amplification in pulsing magnitude that can be achieved by designing a feed system so that acoustic resonance is achieved at the exciter frequency. An example of the design and its calculated performance is shown in Figure 9. The system shown is an example of excitation by an active pressure fluctuator coupled with "organ-pipe" resonance as illustrated in Figure 8a. Wylie presents the results of two methods used to theoretically predict the performance of the system shown. These methods are extensively presented in the textbook by Streeter and Wylie on Hydraulic Transients (Reference 27).

In the example shown in Figure 9, Wylie calculated that for a piston displacement amplitude of $15 \mathrm{cc}$ the effect of pulsating the flow is to increase the erosion level 3.1 times while expending only 1.35 times the power. (The calculation assumes $\mathrm{n}=2.75$ in Equation [1]).

The method proposed by Wylie should be practically achievable in the high pressure jet systems where the exciting piston device can be installed, for example, in applications above ground. Installation of the exciter could be assumed to be above ground for the deep-hole drilling applications, but the long drill pipe leads to resonant frequencies less than $1 \mathrm{~Hz}$. The most pertinent feature of the system proposed by Wylie for the deep-hole drilling application is the importance that should be given to achieving a resonant system.

Nebecker: Figure 10 illustrates, schematically, an active valve system (similar to the general method shown in Figure $8 \mathrm{~b}$ ) that has been developed by Scientific Associates to modulate jet flow at frequencies of $5,000 \mathrm{~Hz}$ and possibly higher (Reference 28). 
The valve is similar to a siren and achieves its high frequencies by the large number of slits in the rotor and stator and their spacing. Although details of this valve were not available in the literature, it is conceivable that the rotor could be driven at high enough speeds to achieve the necessary rate of oscillation. However, since the principal goal of the present study was to uncover or develop passive concepts, the Nebecker valve was omitted from further considerations.

\section{Galle and Woods: Galle and Woods in the Reference 29} patent describe several methods of oscillating the bottom hole pressure. They want to achieve an intermittent increase in bit force on the hole bottom followed by a suction force designed to reverse the pressure differential across the hole bottom and thus improve bottom cleaning. Accompanying such action, the jets used for cleaning necessarily pulsate. The frequency of operation for the various schemes described is approximately $100 \mathrm{~Hz}$. All of the concepts described require no energy source except that extracted from the fluid flow and have no moving parts. Oscillation is achieved with fluid amplifier devices similar to the type shown in Figure $8 \mathrm{c}$ and utilize additional resonant chambers and appropriate passage lengths to couple the fluid amplifier to the fluid in the hole bottom. Figure 11 illustrates one concept utilizing the fluid amplifier as taken from Reference 29.

Since the frequency of all of the devices described in Reference 29 are approximately 100 times lower than the frequencies needed to structure a jet, these devices were not considered further. Furthermore, none of the schemes used to achieve pulsation are capable of alteration to achieve the high frequencies needed. These devices, which are apparently being developed by the Hughes Tool Company, are of interest for cavitating jets if they are successful. That is, the large reduction in pressure at 
the hole bottom during the low pressure cycle will decrease the operating cavitation number during this portion of the cycle and therefore increase the degree of cavitation and thus the erosion. Also such low frequencies should not alter the performance of superimposed high frequency pulsating jets.

Fülöp and Rókár: Figure 12 illustrates the incorporation of a "fluid whistle" in the jet supply passage so as to produce ultrasonic pressure waves in the jet exhaust. This invention, as described in Reference 30 , is an example of the type shown in Figure $8 \mathrm{e}$ that has been applied specifically to conventional mechanical bits for deep-hole drilling and is an excellent example of the type of passive device of particular interest in the present study. However, the reason for desiring high frequency pulsation is different.

The device is directed at improved drilling by augmenting the mechanical bit cutting with ultrasonic pressure fluctuations at the rock face without the requirement to provide acoustic transducers energized from an additional power source. Furthermore, there is no mention of improved jet cutting action or cavitation erosion as a motivation for the device. In fact, Reference 30 makes no reference to cavitation, even though for some operating conditions cavitation should be expected to occur within the resonant chamber and affect its performance.

Although the device described in Reference 30 is probably not an optimum configuration to accomplish passive pulsation of a cavitating jet, the device incorporates some of the principles and general features of concepts envisaged as promising at the outset of the present study. The most important principle not recognized or incorporated in the Reference 30 device was the jet structuring phenomenon reported in Reference 22 and discussed in an earlier section of this report. The frequency suggested for the Fülöp "whistle" is that corresponding to the 
natural frequency of the rock being drilled. Furthermore, the non-symmetric (off-center) element used in the device will inhibit structuring of the jet for enhanced cavitation.

The foregoing pulsing concepts or devices are the most pertinent ones found in the literature that have been proposed for deep-hole drilling. Except for the general work by Wylie (Reference 26) the other devices have been fabricated and developed to some extent. Only the "underwater whistle" concept described in Reference 30 is both passive and operates in the frequency range of principal interest in the present study. 
III. RECENT DEVELOPMENTS IN SELF-SUSTAINED FLUID OSCILLATORS

The principles involved in the oscillations that occur when thin jets are blown against the lip of an organ pipe or across the mouth of a Helmholtz resonator have been discussed and understood for over a century. For examples see Sondhauss (31), Helmholtz (32) and Rayleigh (33). However, self-sustaining fluid oscillations that were observed and discussed by Sondhauss and Rayleigh of the type that occur in a "bird whistle" were not so clearly understood. A "bird whistle" is made by exhausting a jet from an orifice into another orifice of about the same size in a plate located a few jet diameters downstream. The space between the upstream orifice and the plate is not closed but open to the atmosphere. Sondhauss found experimentally that the frequency of the tone produced depended only on the ratios (jet velocity/jet diameter) and (orifice to plate distance/jet diameter); that is,

$$
\left(\frac{\mathrm{Ed}}{\mathrm{U}}\right)=\mathrm{F}\left(\begin{array}{l}
\mathrm{L} \\
\mathrm{d}
\end{array}\right)
$$

The parameter $(f d / U$ ) is the Strouhal number which is seen to have a unique value for a given geometry.

It is now known that the "bird whistle" is a member of a large class of self-sustained oscillations which occur when shear flows (flows with very large velocity gradients) impinge on downstream solid surfaces. Such flows were reviewed and discussed recently (1979) by Rockwell and Naudascher (Reference 34). Examples of impinging shear layer flows are shown in Figure 13 reproduced from Reference 34. A11 of these flows produce tone responses characterized by 
Equation [10]. The oscillation is maintained through acoustic feedback (to the jet source) of a pressure spike generated by vortex impingement on the downstream body. This pulse at the jet source initiates a following vortex that translates downstream at a speed less than the jet velocity to impinge again and repeat the cycle. Higher mode numbers exist at near integer multiples of the fundamental frequency where increasing numbers of vortices are present between the source and the impacted boundary. Figure 14, reproduced from Reference 34, illustrates various types of vortex impingement on the downstream edge of a rectangular cavity such as shown in the right hand column of Figure 13.

For the "bird whistle" jet-hole oscillator the Strouhal number, $f d / U$, which defines the tone of the device is in the range of $0.2-1.0$, depending on the spacing and the mode number. It is in this range of Strouhal numbers, particularly in proximity to a value of 0.3 , that Crow and Champagne's experiments (Reference 22) with forced excitation of round jets show the remarkable change in jet structuring that has been mentioned previously. The observation that jets are sensitive to excitation was a subject of much interest to $19^{\text {th }}$ century scientists; for example, Leconte discussed the phenomena in Reference 35 in 1858. The experiments reported in Reference 22 show conclusively that well defined discrete vortices of the type shown in Figure 14 are formed on round jets only when the jet is excited near the Strouhal number of 0.3 or when this Strouhal number arises as the subharmonic during excitation at $s_{d}{ }^{*}=0.6$. These results have recently been confirmed by others; for example Moore in Reference 36 and Kibens in Reference 37.

Once the jet structuring phenomenon is recognized, it follows that self-sustaining fluid oscillators designed to pick up the harmonic structure of the jet and feed it back must be possible. 
The present investigation began with just that search in mind. It was conjectured that the "bird whistle" described by Rayleigh was just such a device. Furthermore, it seemed likely that if the space between the orifices was enclosed, so as to form a Helmholtz oscillator with a natural frequency tuned to the vortex interaction frequency, that the "gain" of the oscillator should increase. An experimental program was therefore planned to study two tandem orifices connected by a chamber whose natural Helmholtz frequency corresponed to a jet Strouhal number of 0.3 .

The envisioned experimental program was greatly reduced when the work of Morel (Reference 38) was uncovered. Reference 38 presents the results of tests in air on a "Jet Driven Helmholtz Oscillator" in which the geometry was varied over a very wide range. This work was very valuable to the present study. The existence of so much pertinent data allowed us to limit our planned experimental program in air and to go forward with some confirming experiments in water and actually to study the device in cavitation erosion against a rock sample.

Figures 15 and 16 are reproduced from Reference 38 and discussed in the following paragraphs.

Figure 15 illustrates the geometry investigated by Morel. Variations were made in the type of input nozzle and its exit diameter, the chamber diameter and length, and the diameter and length of the cylindrical exit nozzle. The jet velocity was varied such that the Mach number range was 0.01-0.15. The principle measurements were the fluctuating pressure within the chamber and the velocity fluctuations in the exiting jet. Velocity was varied until a peak chamber response was observed. Over one thousand such distinct peaks were observed. In general, at least three peaks (corresponding to the first three modes) were observed for all configurations with some configurations showing 
some response up to the seventh mode. The strongest responses were always for either the first or second mode.

Figure 16 from Reference 38, shows the measured chamber response in terms of the rms pressure fluctuation/jet dynamic pressure, $p^{1} / q$, for the nozzle configuration shown in Figure 15. The influence of the downstream/upstream nozzle diameter ratio, $D_{2} / D_{1}$, and the chamber length/upstream nozzle diameter ratio $L / D_{1}$, for the chamber, responding in the second mode is depicted. The maximum response for this type of configuration in the second mode occurred for $D_{2} / D_{1}=1.2$ and $L / D_{1}=1.6$ and was 3.92 . This rms value corresponds to a (sine wave) amplitude 5.5 times the dynamic pressure of the jet. The jet flow exiting from the chamber pulsated at the same frequency as the chamber with an exit rms velocity fluctuation near the wall of the nozzle of about 60 percent of the steady velocity. Velocity fluctuations downstream of the nozzle exit were not reported, but in view of the experiments reported in Reference 22, the downstream velocity fluctuations should also be large. The achievement of such large pulsations in the exit flow with this simple passive oscillator proves the existence of a device only imagined by the authors at the outset of the present study.

As pointed out and discussed further in the following section it may be observed from the data presented in Reference 38 that maximum response occurs for values of $L / D_{1} \approx 0.8 \times$ mode number and when the downstream orifice diameter is about 20 percent greater than the jet diameter created by the upstream nozzle or orifice. Furthermore, it may be deduced that the optimum response for all modes occurred at a nominal value of the Strouhal number based on diameter of 0.6 rather than 0.3 as would be expected from most of the results of Reference 22 . This result may be explained by the observation in Reference 22 
that unusually large jet expansions occurred when the jet was excited at $s_{d}^{*}=0.6$; apparently caused by the growth of the subharmonic $\left(S_{d}^{*}=0.3\right)$ vortices.

Based on the results of the foregoing discussion, four acoustic oscillators of the general type shown in Figures $8 d$ and $8 e$ were preliminarily designed and selected for further study. The concepts are described in the following sections. 
IV. DESCRIPTION OF PULSING CAVIJET ${ }^{\circledR}$

CONCEPTS SELECTED FOR FURTHER STUDY

\section{A. Organ-Pipe, Plain CAVIJET}

The simplest concept selected for further study is shown in Figure 17. This concept should achieve peak acoustic resonance when a standing wave forms in the "organ-pipe" chamber created by the upstream area-change and the nozzle contraction and whose frequency is near the critical jet structuring frequency. The exact frequency for peak resonance is dependent on the end impedances; these impendances depend on the various area ratios. For instance, if the upstream contraction ratio, $\left(D_{S} / D\right)^{2}$, is large and the exit nozzle contraction ratio, $(D / d)^{2}$, is also large, then the first mode natural frequency of the chamber will occur when the sound wave length is approximately four times the chamber length $\mathrm{L}$. Since the "organ-pipe" chamber will be excited to some degree for all flow rates because some energy is present for all frequencies, a peak resonance should occur when the nozzle Strouhal number is near the critical value $s_{d}^{*}=0.3$ described in Reference 22. Thus, for preliminary design purposes, the length of the "organ-pipe" concept shown in Figure 17 is defined by:

$$
L / d=K_{N}\left(\frac{1}{S_{\mathrm{d}^{*}}^{*}}\right)\left(\frac{c}{U}\right)=\frac{K_{N}}{M S_{\mathrm{d}^{*}}^{*}}
$$

where,

$$
\begin{aligned}
K_{N}=F\left\{N,\left(\frac{D S}{D}\right)^{2},\left(\frac{D}{d}\right)^{2}\right\} & \approx \frac{2 N-1}{4} \text { for }\left(\frac{D S}{D}\right)^{2} \text { and }\left(\frac{D}{d}\right)^{2} \gg 1 \\
& \approx \frac{N}{2} \text { for }\left(\frac{D S}{D}\right)^{2} \gg 1, \text { but }\left(\frac{D}{d}\right)^{2} \approx 1
\end{aligned}
$$

$\mathrm{N}=$ mode number of pipe

$\mathrm{S}_{\mathrm{d}}{ }^{*}=$ critical Strouhal number, $\mathrm{fd} / \mathrm{U} \approx 0.3$

$c=$ sound wave speed

$\mathrm{U}=$ jet speed

$\mathrm{M}=\mathrm{U} / \mathrm{c}=$ Mach number 
The upstream and downstream contraction ratios of 2 and 4 are shown as typical values and should be optimized in a future program if the concept is determined to be feasible.

The configuration shown in Figure 17 was tested in air for a fixed chamber $25 \mathrm{~mm}$ ( $1 \mathrm{in.}$ ) in diameter and $57 \mathrm{~mm}$ (2.25 in.) long with orifice diameters of 3.13 and $6.25 \mathrm{~mm}(0.125$ and $0.25 \mathrm{in.)}$. The results of these tests are described in a following section.

B. Tandem-Orifice, Helmholtz Resonator ("Pulser")

The simplicity of the jet-driven Helmholtz resonator, and the magnitude of the jet pulsing as measured in the experiments reported by Morel in Reference 38 make this a very promising concept for deep-hole drilling.

The concept (henceforth referred to as " Pulser " for brevity) is shown in Figure 18 as being fed by an "organpipe " supply. However, such a supply is not required to achieve large oscillations in the jet and is shown to indicate that the performance might be improved by also achieving resonance in the supply pipe.

The dimensions of the preliminary design shown in Figure 18 are selected based on the peak performance achieved in air and presented in Reference 38. Reference 38 shows that strong oscillations may be achieved over a wide range of configurations as shown in Figure 19.

The principal of operation of the "Pulser " nozzle for peak performance is to match the chamber Helmholtz frequency to the frequency of arrival (at the downstream orifice) of the structured ring vortices passing through the chamber. In the experiments carried out and reported in Reference 38. 
the frequency of strong oscillations for a given configuration was always near the natural Helmholtz frequency of the chamber and occurs at a finite number of discrete jet velocities given by the following equation:

where,

$$
\mathrm{U}=\frac{\mathrm{fL}}{\mathrm{S}_{\mathrm{L}}}{ }^{*}
$$

$$
\begin{aligned}
& S_{L}^{*}=(N-0.25-\mathrm{Lf} / \mathrm{C}) \mathrm{U}_{\mathrm{c}} / \mathrm{U} ; \mathrm{N} \text { (mode number) }=1,2,3 \ldots \\
& \mathrm{U}_{\mathrm{C}}=\text { vortex convective velocity (See Fig. 5, Reference 38). }
\end{aligned}
$$

Although mode numbers as high as 6 were observed for some longer configurations tested, the first three (and usually the first or second) mode gave the strongest response. The range of Strouhal numbers (based on diameter, $d_{1}$ ),$f d_{1} / U$, for strong excitation varied from $0.2-1.0$. The largest pulsations occured in the first two modes with $\mathrm{L} / \mathrm{d}_{1}$ approximately 0.8 and 1.6 , respectively. For these best cases the value of $d_{2} / d_{1}$ was 1.2 .

An interesting empirical observation obtained from the data presented in Reference 38 is that the optimum value of $\mathrm{L} / \mathrm{d}_{1}$ (at least for the configurations described) is approximately $0.8 \mathrm{~N}$. Combining this value with Equation [12] results in the observation that the strongest oscillations occur for each optimum design at a Strouhal number based on diameter approximately equal to $0.6(0.55$ to 0.65$)$. Apparently the jet within the Helmholtz cavity structures most favorably at $\mathrm{s}_{\mathrm{d}}{ }^{*}=0.6$ rather than $\mathrm{s}_{\mathrm{d}}{ }^{*}=0.3$ as might first be expected. However, Reference 22 does note that excitation at $s_{d}^{*}=0.6$ causes a strong $s_{d}^{*}=0.3$ subharmonic that results in an abrupt widening of the jet. This effect may be the reason for maximum response in the Helmholtz oscillator occuring at 
at $\mathrm{S}_{\mathrm{d}}{ }^{*}=0.6$ rather than 0.3 .

The frequency of the cylindrical Helmholtz oscillator shown in Figure 18 for large values of $\forall / d_{1}{ }^{3}$ (where $\forall=$ cavity volume) and $d_{1} / d_{2} \approx 1$ is approximately given by the following equation:

$$
f=\frac{c}{2 \pi} \sqrt{\frac{\pi d_{1}}{4 \forall}} .
$$

or in terms of the length $L$ and the maximum diameter $d_{T}$ :

$$
f=\frac{c}{2 \pi} \frac{1}{d_{T}} \sqrt{\frac{d_{1}}{L}} .
$$

In terms of the nozzle Strouhal number $S_{d}$,

$$
\frac{d_{T}}{d_{1}}=\frac{1}{2 \pi S} \sqrt{\frac{d_{1}}{L}} \frac{c}{U} .
$$

Substituting the approximate critical values of $\mathrm{s}_{\mathrm{d}}{ }^{*}=0.6$ for a nozzle having negligible jet contraction, and $L_{1} / d_{1} \approx 0.8 \mathrm{~N}$, with Mach number, $M=U / c$, gives:

$$
\frac{\mathrm{d}_{\mathrm{T}}}{\mathrm{d}_{1}} \approx \frac{0.3}{\mathrm{M} \sqrt{\mathrm{N}}}
$$

Equation [16] results in a value of $d_{T} / d_{1} \approx 3$ for a first mode oscillator $\left(L / d_{1} \approx 0.8\right)$ operating at a jet speed in water of $150 \mathrm{~m} / \mathrm{s}(500 \mathrm{fps}, \mathrm{M} \approx 0.1)$. If the jet speed is increased to a Mach number of 0.2 the value of $d_{T} / d_{1}$ reduces to 1.5 . Such a low value of $d_{T} / d_{1}$ results in a cavity volume so small compared to $d_{1}{ }^{3}$ that the approximation to the resonance frequency given by Equation [16] is certainly not valid. Furthermore, it is reported in Reference 38 that strong oscillations were not achievable for $M>0.1$. Thus, the "Pulser" nozzle may be limited in application to jet speads less than about $150 \mathrm{~m} / \mathrm{s}$ (49.2 fps). 
Even at a jet speed of $150 \mathrm{~m} / \mathrm{s}$ ( $492 \mathrm{fps}$ ), the cavity volume is only $5.4 \mathrm{~d}_{1}{ }^{3}$ and, as pointed out in Reference 38 the nozzle interaction with the feed pipe must be included in a final design procedure.

Although the "Pulser " nozzle may not be found to be suitable as a terminal pulsing device for nozzle pressure drops greater than about $13.8 \mathrm{MPa}(2,000 \mathrm{psi})$, pressures less than $13.8 \mathrm{MPa}$ are used in current drilling practice. For higher jet exit speeds it may be possible to locate the "Pulser" oscillator upstream of a third exit or terminal nozzle and operate the oscillator at a lower Mach number. Such a scheme, which has the advantage of also operating at higher cavitation numbers than the exit nozzle, is described in a subsequent section.

C. Extended Nozzle-Orifice, Helmholtz Resonator ("Laid-Back-Pulser")

The previous section points out that the "Pulser" nozzle should be immediately applicable as a passive pulsating nozzle when the nozzle pressure drop is $13.8 \mathrm{MPa}(2,000 \mathrm{psi})$ or less and the effect of cavitation within the resonator chamber can be tolerated.

One of the most used extended nozzle bits is the 3-cone roller bit. The geometry of one of the extended nozzle arms for this type of bit is shown in Figure 20. Clearly, it can become impractical in the limited room available in the nozzle arm to provide the desired cavity volume in a "Pulser" configuration, particularly for larger nozzle diameters. A possible method of achieving the desired pulsing flow, shown in Figure 21, is referred to as the "Laid-Back-Pulser". The necessary cavity volume to give the proper frequency is provided partly by the volume surrounding the nozzle that extends into the chamber. The length, $L$, from the exit of the 
upstream nozzle to the orifice exit is retained as approximately equal to $0.8 \mathrm{~N}$ and the total volume of the cavity may be expressed for a simple Helmholtz oscillator as

$$
\frac{\forall}{d_{1}^{3}} \quad \frac{1}{16 \pi}\left(\frac{c}{U}\right)^{2} \frac{1}{S_{d}^{2}}=\frac{1}{16 \pi\left(M S_{d}\right)^{2}}
$$

For the case of $\mathrm{M}=0.1$ and $\mathrm{S}_{\mathrm{d}}{ }^{*}=0.6$ the optimum value of $\left.\forall / d_{1}{ }^{3}\right)$ is approximately 5.5. Figure 21 *illustrates a "Laid-Back Pulser" suitable for installation in mechanical bits where the maximum nozzle outside dimensions are constrained.

Experiments in air on such nozzles are presented in a subsequent section.

D. Jet Driven Helmholtz Oscillator Supply to Plain CAVIJET ("Pulser-Fed" CAVIJET)

As discussed in the previous sections, "Pulser " oscillators used as the primary exit nozzle have at least some preliminary limitations. These are:

1.) Although there is no obvious fundamental reason, Reference 38 states that strong resonance was not achievable at Mach numbers greater than about 0.1 . The most apparent reason is that the value of $\forall / d_{1}{ }^{3}$ becomes small for increasing Mach numbers and much greater care must be given to acoustic interactions with the supply pipe and hydrodynamic interactions with the outer chamber walls.

2.) For the strong resonances desired for maximum pulsation in the exit flow the pressure fluctuations within the chamber can reach peak values of 4 or 5 times the jet dynamic pressure. During the negative portion of the cycle the local pressure may drop below vapor pressure (depending on the operating conditions) and cavitation will occur 
within the chamber. Such cavitation must alter the acoustic performance of the oscillator and has the potential to damage the chamber or exit orifice.

3.) The peak performance of the "Pulser" configurations defined by Equation [16] occurs at a nozzle Strouhal number of approximately 0.6 rather than 0.3 , the "ideal" value for jet structuring. Furthermore, the exiting flow probably does not have a clean shear layer as it leaves the exit nozzle and therefore is not structured into the discrete vortices established within the jet.

The foregoing remarks lead immediately to a configuration which alleviates or minimizes all of these problems. This configuration is shown in Figure 22 and is referred to as the "Pulser-Fed" CAVIJET.

If the upstream "Pulser" is designed to operate at $\mathrm{s}_{\mathrm{d}}{ }^{*}=0.6$ and excites the downstream CAVIJET at $S_{d}{ }^{*}=0.3$, it follows that

$$
\frac{d_{1}}{U_{1}}=2 \frac{d_{e}}{U_{e}},
$$

and from the continuity equation

$$
d_{1}^{2} U_{1}=d_{e}^{2} U_{e}
$$

Therefore,

$$
\begin{aligned}
& \frac{U_{1}}{U_{e}}=(2)^{-2 / 3}=0.63, \text { and } \\
& \frac{d_{1}}{d_{e}}=(2)^{1 / 3}=1.26
\end{aligned}
$$


In such a system the Mach number of the exit jet will be 1.59 times greater than the "Pulser" oscillator Mach number, thus extending the known acceptable jet exit speed to $263 \mathrm{~m} / \mathrm{s}$ (866 fps) or pressure drops of $34.4 \mathrm{MPa}(5,000 \mathrm{psi})$.

It is of interest to estimate the steady cavitation number at the "Pulser" exit in terms of the operating cavitation number at the CAVIJET nozzle exit. The following analysis is keyed to Figure 22 and makes the following assumptions:

o The pressure loss between sections 0 and 3 is $\mathrm{K}_{\ell}\left(\frac{1}{2} \rho \mathrm{U}_{2}{ }^{2}\right)$ where $\mathrm{K}_{\ell}$ may be obtained from momentum and continuity considerations to be

$$
\mathrm{K}_{\ell}=\left[1-\left(\frac{\mathrm{d}_{2}}{\mathrm{D}_{2}}\right)^{2}\right]^{2} \text {. }
$$

Thus, no loss is assumed within the "Pulser" cavity.

- $\quad \frac{1}{2} \rho \mathrm{U}_{\mathrm{O}}^{2} \ll \mathrm{p}_{\mathrm{O}}, \frac{1}{2} \rho \mathrm{U}_{3}^{2} \ll \mathrm{p}_{3}$

- $\mathrm{d}_{1}=\mathrm{d}_{2}, \mathrm{p}_{1}=\mathrm{p}_{2}, \mathrm{U}_{1}=\mathrm{U}_{2}$

Application of the energy equation between sections 1 (or 2) and the exit plane gives:

$$
\mathrm{p}_{1}+\frac{1}{2} \rho \mathrm{U}_{1}{ }^{2}-\mathrm{K}_{\ell}\left(\frac{1}{2} \rho \mathrm{U}_{1}{ }^{2}\right)=\mathrm{p}_{\mathrm{a}}+\frac{1}{2} \rho \mathrm{U}_{\mathrm{e}}{ }^{2} .
$$

Using the definition of cavitation number,

$$
\sigma_{1}=\frac{p_{1}}{\frac{1}{2} \rho U_{1}{ }^{2}}=\frac{p_{a}}{\frac{1}{2} \rho U_{e}{ }^{2}}\left(\frac{U_{e}}{U_{1}}\right)^{2}+\left(\frac{U_{e}}{U_{1}}\right)^{2}-1+K_{l} \text {, }
$$

or since $\quad \sigma_{e}=\frac{p_{a}}{\frac{1}{2} \rho U_{e}^{2}}=\frac{p_{a}}{q_{e}}$.

then

$$
\sigma_{1}=\left(1+\sigma_{e}\right)\left(\frac{U_{e}}{U_{1}}\right)^{2}-1+k_{l} \text {. }
$$


Using, from Equation [20], $\frac{U_{e}}{U_{1}}=1.59$, we finally obtain:

$$
\sigma_{1}=2.53\left(1+\sigma_{e}\right)-1+k_{l} .
$$

Thus, the operating cavitation number at the "Pulser"! oscillator is $1.53+\mathrm{K}_{\ell}$ even when the jet cavitation number is zero. Cavitation within the oscillator is therefore greatly reduced and probably eliminated for most practical conditions.

Now in this "Pulser-Fed" CAVIJET concept the CAVIJET is being " cleanly" excited at its critical Strouhal number. If the excitation magnitude is sufficient, the flow exiting from the CAVIJET should be structured into discrete vortex rings. Such rings should result in a higher incipient cavitation number than for a non-structured jet.

The important disadvantage associated with the Pulser-Fed CAVIJET is the energy loss term, $\mathrm{K}_{\ell} \mathrm{q}_{2}$, in Equation [22]. The value of $\mathrm{K}_{\ell}$ is determined from the momentum equation to be $\left(1-\mathrm{d}_{1}{ }^{2} / \mathrm{D}_{2}{ }^{2}\right)^{2}$. This loss may be then stated as

$$
\frac{p_{0}-p_{3}}{q_{e}}=\left[1-\left(\frac{d_{1}}{D_{2}}\right)^{2}\right]^{2}\left(\frac{U_{1}}{U_{e}}\right)^{2}
$$

For the preliminary design selection of $\left(U_{1} / U_{e}\right)=0.63,\left(U_{1} / U_{e}\right)^{2}=$ 0.4 and

$$
\frac{p_{e}-p_{3}}{q_{e}}=0.4\left[1-\left(\frac{d_{1}}{D_{2}}\right)^{2}\right]^{2}
$$


If satisfactory operation of this concept cannot be achieved for values of $d_{1} / D_{2}>\frac{1}{2}$, where

$$
\frac{\left(p_{0}-p_{3}\right)}{q_{e}} \leqslant 0.225,
$$

the loss in overall efficiency is probably not acceptable.

Some experiments in air were carried out for this Pulser-Fed CAVIJET and are described in the following section. 


\section{EXPERIMENTS}

A series of experiments were conducted to examine those points essential to assessing which of the concepts described in the previous section were the most promising. These experiments are described and the results obtained are presented and discussed in the following sections.

\section{A. Jet Cavitation From A Plain CAVIJET Nozzle Vith Forced Supply Excitation}

The phenomenon reported in Reference 22, that air jets which were harmonically excited became structured into discrete ring vortices at a preferred excitation frequency, was the principle impetus for the present study. It was postulated that such structured fluid jets should cavitate more readily than unstructured jets and thus should have increased erosion intensity, particularly in the deep-hole drilling application. In order to confirm this postulate, an experiment in water similar to the Reference 22 experiment in air was carried out.

\section{Description of Apparatius}

The test loop shown in Figure 23 was assembled by Professor A.T. Ellis in his laboratory at the University of California at San Diego. This recirculating test loop supplied flow through a $6.3 \mathrm{~mm}$ ( $0.25 \mathrm{in.)}$ diameter plain CAVIJET nozzle discharging into a $15 \mathrm{~cm}(6 \mathrm{in.})$ diameter by $30 \mathrm{~cm}$ (12 in.) long high pressure chamber with a viewing port at each end of the chamber. The jet impacted a flat plate located $10 \mathrm{~cm}(4 \mathrm{in}$,$) from the jet$ exit. The flow from the test chamber discharged through a valve (A) to the $0.43 \mathrm{~m}^{3}\left(16 \mathrm{ft}^{3}\right)$ spherical settling chamber. A small vacuum pump was connected to this chamber for degassing the water if desired, A $12 \mathrm{~kW}$ (15 hp), $5.5 \mathrm{MPa}$ ( $800 \mathrm{psi}), 1.261 / \mathrm{s}$ (20. $\mathrm{gpm}$ ) positive displacement pump was connected to the settilng 
chamber and returned the flow through a valve (B) to the test chamber through the CAVIJET nozzle. An $0.08 \mathrm{~m}^{3}\left(3 \mathrm{ft}^{3}\right)$ spherical accumulator was located as shown in Figure 23 to attenuate the low frequency $(30 \mathrm{~Hz})$ pump impulses supplied to the system. The bypass line and valve allowed control of the discharge (velocity) through the CAVIJET nozzle and the valves denoted $A$ and $B$ allowed control of the ambient pressure and thus the test cavitation number. All piping was $2.5 \mathrm{~cm}$ ( 1 in.) in diameter.

Preliminary tests with untreated tap water and with water after slow recirculation under vacuum showed no significant change in the test results and most tests were thereafter carried out with untreated tap water.

A pipe "tee" was located $25 \mathrm{~cm}$ (10 in.) upstream of the CAVIJET nozzle and a lightweight piston was installed in the leg of the "tee." This piston was connected to the output shaft of a $4 \mathrm{~kW}$ ( $5 \mathrm{hp}$ ) "shake table" oscillator capable of oscillating the piston harmonically over a range of frequencies from 5 $5,000 \mathrm{~Hz}$. The piston displacement amplitude was picked up electronically and displayed on an oscilloscope.

Static pressure was measured upstream and downstream of the test chamber with laboratory Bourdon type gages and dynamic pressure was measured in the wall of the supply tube $16.5 \mathrm{~cm}$ (6.5 in.) upstream of the nozzle exit and $3.2 \mathrm{~cm}(1.25 \mathrm{in.})$ downstream from the nozzle exit and on the jet centerline with high frequency $(50,000 \mathrm{~Hz})$ piezoelectric pickups.

Cavitation was detected both visually and audibly (with the crystal pickup in the chamber) and by the light scattering technique illustrated schematically in Figure 24. Light from the 1 millivolt helium-neon laser passed through the jet 
centerline $6.3 \mathrm{~mm}(0.25 \mathrm{in.})$ downstream of the nozzle exit. The laser light was aligned with an electron multiplier type photocell whose opening was covered except for a $3 \mathrm{~mm}(0.12 \mathrm{in.})$ hole through which the laser light passed when no cavitation was present in the jet. Any cavitation bubbles formed in the jet and passing through the laser beam caused the light to scatter and deflect the beam. The output from the photocell was supplied to an oscilloscope so that cavities formed in the jet were detected as "spikes" on the oscilloscope screen. The cavitation detector output could be displayed on the same screen as the piston displacement and pressure pickups. For example, Figure 25 shows the piston displacement and laser cavitation detector outputs during operation near the critical jet Strouhal number (jet speed $-30 \mathrm{~m} / \mathrm{s}(100 \mathrm{fps})$ ).

It was discovered during the test program that the response of the mechanical driver decreased rapidly above about $3,000 \mathrm{~Hz}$ and it was not possible to achieve an oscillation of the pressure of even a few percent of the steady supply pressure level. The mechanical driver was then replaced by a magnetostrictive oscillator developed for the tests which was capable of providing pressure oscillations of $0.05 \mathrm{MPa}(7.5 \mathrm{psi})$ which is two percent of the maximum steady pressure tested (approximately $2.4 \mathrm{MPa}$ (350 psi)) at frequencies as high as $10,000 \mathrm{~Hz}$.

2. Experimental Results

The experimental results may be summarized as confirming the postulate that a CAVIJET, excited with approximately 2 percent of the steady level, structures itself into easily visible (when cavitating) discrete vortex rings when the frequency of excitation corresponds to a strouhal number, based on the CAVIJET orifice diameter, of 0.46 . Since the contraction coefficient of the 
CAVIJET nozzle is known to be 0.75 , the Strouhal number based on $j$ et diameter $\left(s_{j}{ }^{*}\right)$ was 0.35 and thus in approximate agreement with the results reported in Reference 22. Furthermore, the incipient cavitation number approximately doubles under this critical condition - the actual amplification depending on the magnitude of the excitation. Figure 25 is an indication of the degree of structuring that occurs at the critical strouhal number. In this figure, taken for the case of $\mathrm{s}_{\mathbf{j}}{ }^{*}=0.35$, $\mathrm{U}_{\mathrm{e}}=30 \mathrm{~m} / \mathrm{s}$ (100 fps), $\sigma \approx 0.5$, the lower spikes, corresponding to passing cavities, align nearly perfectly with the upper trace which indicates excitation amplitude. Observations at frequencies approximately $\pm 15 \%$ from the critical frequency shown result in totally uncorrelated cavity spikes with reduced amplitude. Any one ranging the frequency control and observing the oscilloscope amplitude is inmediately impressed with the remarkable correlation achieved near the critical frequency.

In preliminary experiments with the mechanical exciter no attempt was made to quantify the correlation observed except by observer judgment. Figure 26 illustrates the results as judged and agreed upon by three observers. The circle points are the observed frequency of near perfect correlation and the upper and lower bars are the bounds beyond which no correlation was evident. Subsequent tests with the magnetostrictive exciter and and a spectrum analyser confirmed the measurements presented in Figure 26.

Figure 27 presents photographs taken through the view port with short duration strobe lighting, with and without input excitation at the frequency for peak correlation. Although some slight cavitation was always visible to the naked eye at the cavitation numbers indicated on the photos for the unexcited 
cases, the cavitation was not always recorded on the photographs. With the excitation indicated on the figure, large discrete ring vortex cavities are clearly apparent in the photographs, whereas such ring vortices do not appear for the unexcited cases.

The excitation for each case corresponded to a jet Strouhal number of 0.35 except for the highest speed case where the frequency was doubled to $s_{j}{ }^{*}=0.70$. The discrete vortex patterns shown in these photographs were not observed if the frequency was varied more than about 10 percent from the value of 0.35 . However, restructuring occurred at a value of $s_{j}^{*}=0.70$ with the spacing between rings approximately halved.

In order to determine if excitation at the critical frequency influenced cavitation inception, the incipient cavitation number was determined for several speeds with and without excitation at the critical Strouhal number. The results of these tests are presented in Figure 28. For a range of speeds (Reynolds numbers) tested, some variation in the incipient cavitation number was observed for both the excited and unexcited cases. Since the general expectation is that the value of $\sigma_{1}$ will increase with increase in the Reynolds number, the variation shown in Figure 28 is not understood and may only be indicative of experimental inaccuracy for determination of $\sigma_{i}$. However, the difference between the values of $\sigma_{1}$ with and without excitation were easily detected. That is, the condition for incipient cavitation without excitation would readily cavitate when. excitation was imposed and with excitation, for a given velocity, the ambient pressure would have to be increased in order to suppress the cavitation.

Figure 28 shows that the value of $\sigma_{1}$ for the unexcited case was increased 1.4 to 1.8 times by excitation at the critical Strouhal number with an amplitude of only $0.02\left(\frac{1}{2} \rho U_{e}{ }^{2}\right)$. It 
was not possible to vary the excitation to values greater than $0.02\left(\frac{1}{2} \rho \mathrm{U}_{\mathrm{e}}{ }^{2}\right)$ at the higher speeds tested because of the characteristics of the exciter. However, when the excitation amplitude was increased to $0.07\left(\frac{1}{2} \mathrm{\rho U}_{\mathrm{e}}{ }^{2}\right)$ for the low speed case shown in Figure 28 (triangular symbol), the incipient cavitation number increased to a value more than twice the value obtained for the unexcited case.

The results of the experiment described in the foregoing paragraphs are particularly important. They confirm that a submerged jet structures itself into well defined ring vortices when excited at a critical frequency and that the incipient cavitation number for this new flow pattern is significantly increased.

B. Self-Excited Oscillator Experiments - Air

"Bench-type" experiments were carried out, using air, to study further each of the most promising oscillators selected and described previously.

1. Test Apparatus

The apparatus for these experiments is shown schematically in Figure 29. A rectangular plenum supplied air to the device being tested. The pressure in the plenum was controlled with a needle valve in the $0.2 \mathrm{MPa}$ ( $30 \mathrm{psig}$ ) air supply line and was monitored with a U-tube manometer. Flow from the test nozzle discharged to the atmosphere and impinged on a flat plate placed normal to the jet's axis at various locations (this "target" was removed for most tests). Perturbations in the jet's axial velocity were surveyed with a $25 \mu$ diameter hot film sensor. The probe was mounted on a motor driven rig (Figure 30) so it could travel slowly along the nozzle's longitudinal axis at 
$1.3 \mathrm{~mm} / \mathrm{s}(0.05 \mathrm{in.} / \mathrm{s})$ or be located anywhere in the jet flow as desired. A microphone was placed nearby to sense the acoustic signatures emitted by the nozzle device.

The electronic signal processing suite is shown in Figures 31 and 32. The raw voltage signal from the Thermo-systems Inc. (TSI Model 1050) hot-film anemometer bridge was fed directly to a TSI Model 1060 RMS Voltmeter, a spectrum analyzer (Unigon Model 256), and an X-Y plotter. The RMS Voltmeter output was also fed to the plotter so that the mean and the root-meansquare anemometer voltages could be continuously recorded as a function of the hot film probe's axial distance from the nozzle. The spectrum analyzer output was viewed on an oscilloscope to detect resonant frequencies in the perturbation velocity; these are manifested as sharp spikes or peaks in the spectra. The microphone output was also fed to the spectrum analyzer and another RMS Voltmeter. Thus, the frequency and intensity of sound pressure levels were also monitored.

2. Mean and RMS Velocities

The anemometer output, $\mathrm{E}$ is a non-linear function of the instantaneous velocity, $u$, past the sensor:

$$
E=a u^{n} \text {, volts }
$$

where $a$ and $n$ are empirically determined constants. For convenience, the root-mean-square of the fluctuating component of the velocity, $u^{\prime}$, is normalized by the temporally averaged velocity, $U$. Hence, the "intensity" of the fluctuations is given by:

$$
\frac{u^{\prime}}{U}=\frac{1}{n} \frac{e^{\prime}}{E}
$$

where $e^{\prime}$ and $E$ are the RMS and mean of the anemometer output 
voltage. Similarly, the local mean velocity has been normalized by the maximum mean velocity in the jet, $U_{e}$, which occurs near the orifice or nozzle exit.

The RMS and mean velocity data are presented as functions of the axial distance from the nozzle exit, $X$, normalized by the exit orifice diameter, $d_{e}$.

\section{Experimental Results}

\section{Organ Pipe CAVIJET}

The jet from an orifice that is fed by a fixed length of pipe was found to oscillate, with moderate intensity, at a fixed frequency. The frequency of the self-excited perturbations correspond to an acoustic wavelength, $\lambda$, that is normally four times the pipe length, L. Several geometries were tested with variations in length, pipe diameter, orifice diameter and velocity. Data from two such cases are presented in Figure 33 . These are typical of the results for peak resonance conditions. In one case $\left(d_{e}=3.2 \mathrm{~mm}(0.125\right.$ in. $\left.)\right)$ the frequency corresponded to $\lambda / L=4.2$ and 4.5 , simultaneous $1 y$ at times. The jet was modulated over a wide range of velocities, but the intensity dropped off when the Strouhal number was too low $(<0.1)$ or too high $(>1)$.

The modulation of the jet axial velocity is very coherent to a distance of about three diameters from the exit. Thereafter, the flow is turbulent with a broad and rather flat spectral distribution. In the coherent range the frequencies of the velocity fluctuation are identical to the audible "whistle" tones sensed by the microphone.

Generally, the intensity of coherent modulation for an organ pipe orifice was a little less than 20 percent of the exit velocity, and downstream turbulent intensities (in the center of the jet) ranged from 10 to 20 percent. The downstream turbulence 
intensity is comparable to levels associated with a nonmodulated jet issuing from an orifice, but low levels, less than a few percent, are typical for the first several diameters with these plain jets.

The effect of the flat plate target was determined to be localized to within one jet diameter distance from the plate. close to the plate the turbulent intensity increased slightly. As long as the plate was more than a few jet diameters from the nozzle, the jet modulation was unchanged.

"Pulser"

Very strong jet modulations are excited by resonances within a sma11 chamber surrounding the jet as was shown by Norel (Ref. 38). As noted earlier, we dubbed this type of selfexcited nozzle - "Pulser". Tests in air were performed to confirm Morel's findings and to investigate the effects of various geometric variations that are of interest for practical applications to deep-hole drilling.

Velocity data for peak resonant condtions with a representative " Pulser "geometry are presented in Figure 34. The nozzle dimensions are shown in the inset. The performance is sensitive to small changes in the diameter of the second orifice, $d_{2}$, as shown by the three curves for mean and turbulent velocities. Nevertheless, in each case a coherent velocity modulation with about 50 percent intensity developed within one diameter of the exit orifice. Downstream, the fluctuations were chaotic and approached the 20 percent level of intensity. The high modulation near the jets' origin caused a rapid mixing and momentum transfer with the ambient fluid as evidenced by the rapid downstream reduction in mean axial velocity (compared to non-modulated jets and the more weakly modulated ones obtained for the organ pipes shown in Figure 33 ). 
The jet Mach number, $M$, for peak resonance was normally 0.08 for the geometries shown in Figure 34 and the frequency of modulation corresponded to a Strouhal number based on the exit diameter $\left(d_{2}\right)$ of about 0.3. Strouhal numbers and Mach number effects on "Pulser " performance will be discussed in a later section.

"Laid-Back Pulser"

For certain applications, the maximum diameter that is available for the "Pulser " type resonant chamber is strictly limited. To increase the chamber's volume without exceeding the critical dimension we tried configurations wherein an enlarged volume is achieved by extending the chamber back around a'lengthened orifice. The geometry is depicted in the inset of Figure 35.

Parameters that were varied while examining this kind of configuration included the exit diameter $\left(d_{2}\right)$, the space between the long orifice nozzle and the exit ( () , and the Mach number.

It was found that no resonance would occur if $l$ was less than $d_{1}$ or greater than $3 d_{1}$ Furthermore, the performance was much degraded when the exit diameter was several percent larger or smaller than $d_{1}$. The data in Figure 35 represent the results with optimum conditions for this type of geometry. The maximum coherent velocity modulation intensity was 30 percent. Hence, this alternative is feasible but not especially attractive.

\section{"Pulser-Fed" Orifice (Diffuser).}

We investigated methods of exciting an exit or terminal nozzle with internal "Pulsers" located upstream. A primary consideration with this concept is to create modulations in a non-cavitating flow region that feeds a cavitating jet; cavitation within the chamber of an elementary (or exit) "Pulser " 
would, conceivably, interfere with the resonance phenomena as discussed in the earlier section describing this concept.

The first concept tested used a diffuser between the " Pulser " section and the exit orifice as shown in the inset of Figure 36. In these tests, the Mach number, exit diameter $\left(d_{e}\right)$ and the length of the straight pipe section between the (fixed) diffuser and the exit orifice ( $l$ ) were varied. The object of the diffuser is to minimize the losses associated with the expansion of the flow between the Pulser section and the exit contraction.

With the best such geometry and conditions (as shown), the coherent velocity modulations were only moderate, having less than 20 percent intensity. Worse yet, when the exit diameter, $d_{e}$, was less than the "Pulser " diameter $\left(d_{2}=d_{2}\right)$ the performance was very much degraded. Hence, this concept was abandoned. We presume that the diffuser walls were interfering with the development of ring vortices downstream of the "Pulser " section or the basic natural frequency of the system was reduced to such a low value by the diffuser that it was not possible to excite smaller exit jets at the critical frequency for structuring.

"Pulser-Fed". Orifice (Sudden Expansion)

strong coherent modulations were achieved when a straight length of pipe was placed between the Pulser section and the exit orifice as shown in Figure 37 . We varied the Mach number, exit diameter $\left(d_{e}\right)$ and connector pipe length $(l)$.

Peak velocity modulations ranged from 30 to 45 percent. They occurred further downstream, more than two jet diameters, than for a simple Pulser. At $X=0$, the rms velocity decreased with increasing velocity or Mach number. Far downstream, say 
at $x / d_{e}=12$, turbulence intensities were remarkably high, typically ranging from 25 to 40 percent.

Performance was degraded if the length, l, was less than about $30 \mathrm{~d}_{e}$, but was nearly independent of length for greater values (in fact, a long piece of vinyl tubing that was bent into a loop between the "Pulser" and the exit nozzle gave similar results). Exit diameters from 68 to 77 percent of $d_{1}$ produced qualitatively similar results. The pressure in the pipe ahead of the exit was nominally 70 percent of the feed pressure ahead of the "Pulser section" for all cases tested.

Resonance was achieved for exit Mach numbers, $M_{e}$, ranging from less than 0.05 to more than 0.15 . The frequencies of strong coherent modulation corresponded to exit jet Strouhal numbers from 0.2 to 1.0. Often, two or three frequencies would coexist.

In spite of the high ( $\approx 30$ percent) power loss that is incurred with this concept, it may be justified where internal cavitation gives rise to problems with a simpler Pulser nozzle. Unfortunately, we did not have time to perform cavitation and erosion tests with this concept in water.

"Pulser" Frequency Coup1ing

The parameters that might affect the design of the resonant chamber geometry in the "Pulser "nozzle have been discussed in previous sections. Briefly, the conditions for peak modulation depend on the interrelationships between the chamber modal frequencies, the jet Strouhal number and the Mach number.

Resonant modulation frequency data for two "Pulser" geometries are presented in Figures 38 and 39 . The only change between the two cases was in the chamber diameter, $d_{T}$. The nozzle with smaller diameter, $\mathrm{d}_{\mathrm{T}}=22 \mathrm{~mm}$ (0.88 in.), was referred to as 
the "HF (high frequency) Pulser" and the other as the "LF Pulser."

The frequency (f) has been normalized with the jet velocity $\left(U_{e}\right)$ and chamber length (L) to obtain a non-dimensional parameter, $S_{L}$ (the product of the jet Strouhal number and the ratio of chamber length to exit diameter). This parameter is significant because resonant modulation should occur at discrete values corresponding to modes of operation. The data show the frequency coupling parameter as a function of the jet velocity or Mach number, M. Where data points overlap or are connected by lines, a particular mode was continuously present when the velocity was varied up or down over that interval. For example, first mode $\left(S_{L}{ }^{*} \approx 0.5\right)$ oscillation was manifest for $M$ from 0.06 to 0.15 for the LF Pulser and to 0.20 for the HF Pulser. A few other isolated oscillations were noted at lower velocity and/or at the second mode.

Lines of constant frequency are labelled with the corresponding ratio of wavelength $(\lambda)$ to chamber length. These were drawn to help illustrate our finding that the exact frequency at a given velocity seems to involve a "trade-off" between coexisting mechanisms. That is, there are specific frequencies (including the chamber's primary natural frequency and others that depend on different length scales in the geometry) which can raise or lower the value of $S_{L}$ when the device is operating near their domain.

For example, in Figure 39, the line for $\lambda / L=28(1150 \mathrm{~Hz})$ lies close to the mode 1 data points in the range $0.06<M<0.08$ and to the two connected data points at $S_{L} \approx 0.3$ and $M \approx 0.11$. Since there is no mode number that fits such a low value of $S_{L}$. it suggests that the nozzle entry length, $\mathrm{L}_{\mathrm{p}}$, is providing an, organ pipe type of oscillation mechanism where $\lambda / L_{p}=3.9$. 
Similarly, in Figure 38 one of the data points in the mode 2 range also lies near a $1200 \mathrm{~Hz}\left(\lambda / L=26 ; \lambda / L_{p}=3.7\right)$ line. Especially strong modulation might be expected to occur at the Mach numbers for concurrence of particular resonant frequencies (fixed $\lambda / L$ 's) and the various modal values of the frequency coupling parameter (e.g., $s_{L}{ }^{*} \approx 0.5,0.9,1.3$, etc ; see Equation [12]). "Pulser" Modulation Amplitude

Velocity modulation intensity, $u^{\prime} / U$, and the square root of the pressure modulation ( $\mathrm{p}^{\prime}$ ) (normalized by the nozzle driving pressure, $\Delta \mathrm{p}), \sqrt{\mathrm{p} / \Delta \mathrm{p}}$ are also shown in Figures 38 and 39. The microphone output ( $p^{\prime}$ ) was uncalibrated, so an arbitrary scale factor was selected for this parameter. The values of $u^{\prime} / U$ were measured at $X / d_{2}=1.0$. The data disclose a strong correlation between the amplitude of coherent velocity fluctuations that evolved in the jet and the audible sound pressure levels emitted by the "Pulser " nozzle when operated with air.

The amplitudes for the $\mathrm{HF}$ and LF Pulser nozzles are nearly the same in the range of Mach numbers over which both were active (0.06 to 0.15$)$. The HF Pulser nozzle exhibited a more pronounced and sharper peak at $M=0.08$. Optimum Mach numbers (in mode 1) for the two designs, calculated with Equation [15], are 0.083 and 0.053 for the HF and LF nozzles, respectively. Perhaps the HF nozzle's greater peak amplitude was caused by a fortuitous matching of the chamber resonance with a feed (organ) pipe type resonance. The feed pipe was present in the tested geometries because we incorporated nozzle parts that were also used in high pressure water experiments where certain characteristics are predicated by the tool holding arrangements. 
C. Self-Excited Oscillator Experiments - Water

The simplest and most promising concept, the Helmholtz oscillator or "Pulser " nozzle was subjected to further exploratory experiments in water to confirm the device's resonance characteristics when operating with internal and external cavitation.

\section{Test Apparatus}

Cavitation inception and pressure modulation tests were carried out in the Hydronautics High Pressure Cell (HPC). Comparative rock erosion tests were run (at very low cavitation numbers) in a small, atmospheric pressure, test facility.

The HPC system is shown in Figure 40. A close-up view showing recently added viewing ports is presented in Figure 41 . The system design and its operational characteristics are detailed in Reference 14. Two PCB Piezotronics $6.4 \mathrm{~mm}$ ( $1 / 4$ inch) diameter Quartz piezoelectric pressure transducers (Model No. 101A04) were mounted on the apparatus, one in the inlet supply pipe feeding the nozzle and the other in the wall of the main pressure vessel as shown in Figure 42. Instrumentation was the same as for the experiments in air. Pressure signals were usually filtered to eliminate low frequency pump induced fluctuations.

A picture of the atmospheric pressure test chamber is shown in Figure 43. This unit's capácity is $0.321 / \mathrm{s}$ (5 gpm) at $6.9 \mathrm{MPa}$ ( 1,000 psi) pressure across each nozzle (conventional, "Pulser", or plain CAVIJET).

\section{Experimental Results}

"Pulser" Frequency Coupling

Data from resonant frequency tests, analogous to tests in air (Figs. 38 and 39), are shown in Figures 44 and 45 . One difference between air and water tests is that the nozzles 
were fed by a long network of hose and pipes rather than a large plenum. In view of this seemingly minor configurational change, it is surprizing that there were so many resonances that occured over the entire range of Mach numbers in modes 2 and 3 , compared to so few in the air tests. Usually two or three distinct spectral spikes coexisted in the pressure transducer signals.

The ambient chamber pressure $\left(p_{a}\right)$ was $6.9 \mathrm{MPa}(1,000 \mathrm{psi})$ for this test sequence, so cavitation was negligible; the jet cavitation number $\sigma=\mathrm{p}_{a} / \Delta \mathrm{p}$, exceeded 1.0 for Mach numbers less than 0.08 .

Pressure Fluctuations

Pressure fluctuations generated by the HF Pulser in HPC tests are shown in Figure 46. The RMS of the signal in the frequency band 1 to $100 \mathrm{kHz}$ was normalized by the nozzle driving pressure, $\Delta p$. Data were obtained from the transducer in the feed pipe $\left(p^{\prime} p\right)$ and the transducer in the chamber $\left(p^{\prime} c\right)$. In one experiment the ambient pressure was maintained at $2.8 \mathrm{MPa}$ (400 psig) while jet Mach number was varied. For this case the chamber pressure fluctuations generally increased with increasing Mach number while feed pipe fluctuations varied inversely with Mach number. We attribute this to cavitation noise effects. The chamber pressures include cavitation generated signatures (broad spectrum noise); the cavitation intensity increases with decreasing cavitation number (increasing Mach number in this case). The pressure signatures in the feed pipe are apparently sensitive to the coherent pressure pulses generated by the Pulser nozzle, but the cavitation noise is not as effectively transmitted back upstream.

Pressure fluctuation measurements in the Pulser nozzle chamber were not made, but it is reasonable to expect the intensities 
here to exceed unity, as reported by Morel (38) for his tests in air. The intensities transmitted back to the feed pipe are thus deemed to be less than ten percent of the internal modulations.

The cavitation effects on the modulating mechanism are addressed in Figure 47. These data for chamber pressure fluctuation are analogous to those in the previous figure, but they are cross plotted against cavitation number for fixed values of Mach number. A curve for a non-modulated, plain CAVIJET nozzle is also shown for comparison. Below the cavitation number for inception $(\sigma \approx 0.6)$ the normalized pressure intensity is seen to rise with decreasing cavitation number for the plain CAVIJET. Conversely, the Pulser generated pressure intensity (composed of both the coherent modulation and broad band cavitation noise) decreases slightly with decreasing cavitation number. So, cavitation apparently does interfere with the modulation mechanism to some degree.

There is reasonable qualitative agreement in the Mach number dependence of the "Pulser's" coherent modulation between air and water tests. The square root of the fluctuating pressure in air (from Figure 39) and water (from Figure 46) are plotted together in Figure 48. Recall that the scale factor for the air measurements was arbitrary. Both data sets exhibit the same trends with local peaks around $M=0.077$ and 0.105 . Cavitation Inception

The cavitation number for inception $\left(\sigma_{i}\right)$ of visible cavitation bubbles in an emerging jet was found to be enhanced by the Pulser's modulating effect as compared to simple CAVIJET nozzles. Data shown in Figure 49 were obtained for similarly sized nozzles (orifice diameter $6.4 \mathrm{~mm}$ ), all with initial 
contraction ratios, $\mathrm{D} / \mathrm{d}_{\mathrm{e}}=4$. The four nozzles were all tested over the same range of velocities; however, temperature was not controlled so the jet Reynolds number range was slightly altered. The jets impinged on a flat plate located at $\mathrm{x} / \mathrm{d}_{\mathrm{e}} \approx 4$. One of the non-modulated CAVIJET nozzles had a centerbody in the orifice. The centerbody diameter was one-half the orifice diameter.

The HF Pulser nozzle clearly exhibited cavitation at significantly higher values of cavitation number. It is surprising that inception for the LF Pulser was not likewise high in view of their similar performance in other respects.

Note that the location for incipient visible bubble cavities was relatively close to the nozzle for the HF Pulser, i.e., within $\mathrm{x} / \mathrm{d}_{\mathrm{e}}<1$. With a plain CAVIJET, on the other hand, inception occurred randomly over the entire length of the free jet and near the surface of the target plate beyond the jet's impinging diameter. With the Pulser nozzle, bubbles were not observed near the target surface until the cavitation number was lowered to values comparable to inception for the plain CAVIJET, For each of these CAVIJET nozzle types, plain, Pulser, and centerbody, the isolated bubbles observed at inception and at lower cavitation numbers appear to be located on the periphery of the jet.

Cavitation Patterns

Typical views of cavitation generated by jets at a moderate cavitation number $(\sigma=0.25)$ are presented in Figures 50,51 and 52. The photographs were made with a single strobe flash with the lighting from the left side. The external edge of the nozzle is highlighted in the upper left portion of each picture. The jet is directed vertically down onto the target plate which is in the frame but not visible. The bright spots are the light reflected by the cavitation bubbles/sheets. In Figures 51 and 52 
the horizontal line of bubbles near the bottom of the frame is on or near the target plate.

Note that the bubble patterns are transitory and somewhat random. However, the heavier cavitation seen for the HF Pulser in Figure 50 is representative of this nozzle's characteristics. It is interesting that there is little evidence of cavitation on the plate's surface in this view as compared to Figures 51 and 52.

Preliminary Erosion Tests

To provide some information on the relative erosivity of a self-excited Pulser type nozzle,tests were performed on three specimens of Berea sandstone in the atmospheric pressure rig. It should be noted that the new Pulser nozzle (the middle nozzle in Figure 43) has consistently outperformed the plain CAVIJET nozzle when demonstrated on specimens of common fire brick; the Pulser hole depth in brick is sometimes less, but the volume removal is usually significantly larger (as great as two times). The cavitation noise generated by the new nozzle is discernably more intense as well.

Eight holes were made in each sandstone specimen, four with the Pulser nozzle and four with the plain CAVIJET. The nozzle exit diameters were both $2.2 \mathrm{~mm}$. The Pulser chamber length and diameter were $3.3 \mathrm{~mm}$ and $12.7 \mathrm{~mm}$, respectively. Nozzle standoff was $\mathrm{X} / \mathrm{d}_{e}=7$ and the holes were drilled normal to the bedding plane. Driving pressure was $6.9 \mathrm{MPa}$ ( 1,000 psi) and the cavitation number was about $\sigma=0.015$.

Results from each specimen were qualitatively similar, but the variance was least in specimen No. 1. These data for maximum depth and volume removal are plotted in Figure 53 as a function of the exposure time. The symbols represent the mean value for four holes and the error bar denotes (twice) the 
standard deviation of the four data. The two jets created nearly identical maximum depths. The volume removal rate was about 20 percent larger for the Pulser nozzle, however.

The most significant conclusion to be drawn from these tests is that the Pulser nozzle was superior to the plain CAVIJET nozzle even at a very low cavitation number. That is, cavitation which must occur within the Pulser chamber did not cause the Pulser erosion capability to be worse than the plain CAVIJET; in fact, it was superior in performance at what should be the worst operating condition. 


\section{SUMMARY OF RESULTS AND ASSESSMENT \\ OF FEASIBILITY}

In order to summarize the results of this study it is useful to address the questions asked at the outset.

1. If a high pressure water jet is excited at its source will there be preferred frequencies at which the jet structures into ring vortices and does the jet then cavitate at higher cavitation numbers?

The tests described herein definitely show that a water jet structures itself into discrete ring vortices when excited near a frequency corresponding to a Strouhal number of 0.3 (and 0.6 ) and the jet incipient cavitation number approximately doubles for an excitation intensity of only $0.02\left(\frac{1}{2} \rho \mathrm{U}^{2}\right)$.

2. Are there existing systems or systems under development which might be adapted in deep-hole drilling to achieve a passive oscillation of the jet flow at frequencies near the frequency required to cause discrete ring vortices to form?

Only a few systems have been uncovered that could apply to deep-hole drilling. A number of patented schemes for causing low frequency $(100 \mathrm{~Hz})$ pressure oscillations at the hole bottom are apparently being developed by the Hughes Tool Company. Such low frequencies are not capable of structuring high speed jets $(>150 \mathrm{~m} / \mathrm{s})$. However the existence of such a low frequency oscillator would not impair the performance of the high frequency $(>7500 \mathrm{~Hz})$ oscillator that is sought and in fact would improve jet cavitation performance during the low pressure portion of the cycle.

The patented ultrasonic "whistle" described in Reference 30 has apparently been tried in deep-hole drilling. The principle 
of operation of this device is similar to the Helmholtz oscillator schemes which were selected as being most promising. However the device as described uses an eccentrically located jet within the resonant chamber and its performance relative to an axisymmetric oscillator is not known.

3. Are there concepts of passive systems capable of providing the jet excitation required to structure the jet and cause large pulsations in the jet flow? If so, do simple experiments at least show the concept to have promise?

Several concepts incorporating a jet-driven Helmholtz resonator similar to the Morel oscillator described in Reference 38 have been preliminarily designed and bench tested both in air and water. Velocity oscillation intensities in the jet of over 60 percent are easily achieved. Furthermore, a concept for making the device small enough to fit into the small extended arms of three-cone roller bits has been shown to cause high pulsations in air experiments. However, initial experiments in water with the oscillator at the nozzle exit has not resulted in the degree of jet structuring observed with active excitation of a plain CAVIJET.

A preliminary design which uses the "Pulser" concept to feed a single CAVIJET nozzle downstream has been shown to produce very high jet pulsation amplitude and should operate cavitation free within the "Pulser." This concept shows promise of providing the same degree of jet structuring as achieved with active excitation. However, time did not permit testing this concept in water. This concept necessarily introduces an additional energy loss in the system which must be studied and minimized before the concept is finally judged. 
4. If a passive system for improving the performance of pressure jet augmented drill bits has been uncovered that shows promise, what is the possibility that such a system can, through further research, be developed into a practical or feasible system?

Although there are a number of areas which require further research to improve performance, particularly in the range of low cavitation numbers, the concepts uncovered are very simple and can be designed to replace conventional nozzles in a11 types of drill bits. Tests conducted in air show that very strong pulsations are achievable in the jet at Mach numbers less than 0.1 so that similar performance should be expected in water at current operating nozzle pressure drops of approximately 13.8 $\mathrm{MPa}(2,000 \mathrm{psi})$, if cavitation within the chamber does not greatly alter the effect. Preliminary simple tests in water show that a Pulser type nozzle has an incipient cavitation index in the jet about twice that of a plain CAVIJET nozzle. Preliminary tests at very low cavitation numbers show the Pulser nozzle to be about 20 percent more erosive than a plain CAVIJET. The latter result is particularly important because it shows superior performance even when the flow within the Pulser chamber must be cavitating severely. Furthermore, it is doubtful that the Pulser nozzle tested is even close to an optimum configuration. Therefore, for operating nozzle pressure drops of approximately 13.8 MPa (2,000 psi) (typical of current drilling practice) it is very probable that pulser nozzle systems can be developed for installation in all types of mechanical drill bits currently in use, with a resulting improvement in drilling performance. The magnitude of improved performance cannot now be estimated and must await further development and trials. 
For nozzle pressure drops greater than $13.8 \mathrm{MPa}(2,000 \mathrm{psi})$ in water, where the Mach number of the jet is greate than 0.1 , air test data indicate that strong pulsations are difficult to achieve in the simple Pulser nozzle configurations studied. However strong oscillations were achieved in air in a modified Pulser fed CAVIJET system corresponding to a Mach number of 0.17 . With further research it is expected that Pulser systems can be designed to produce strong oscillations at pressure drops substantially higher than $34.5 \mathrm{MPa}(5,000 \mathrm{psi})$.

The only factor that may compromise the successful development of practical Pulser systems is nozzle erosion caused by either cavitation or solid particle abrasion or both. However, it is anticipated that with proper nozzle design and material selection Pulser systems can be designed to have an adequate life.

The following section sets forth a research and development program which, if pursued with reasonable effort, should result in the introduction of worthwhile "low pressure" Pulser nozzle systems by 1984. Furthermore, this program should provide worthwhile "higher pressure" Pulser systems within three years and optimum systems by 1985 . 
VII. LONG RANGE RESEARCH AND DEVELOPMENT PROGRAM

It is not possible to prescribe in detail the research and development effort required to achieve a specified improvement in drilling rate through the use of the Pulser type nozzle systems uncovered in this study. However, it is reasonable to list the major questions that must be answered as completely as possible by the $R \& D$ program and to suggest a means for acquiring the needed information through theory and analysis, laboratory experiment, and field trials, and how these activities must interact to at least contribute significantly to the answers. Such a list of questions is presented in the following paragraphs along with suggested means of acquiring answers. Finally, Figure 54 shows the interaction between the various $R$ \& $D$ activities which should result in satisfactory design procedures and hardware available for commercial use.

1. What tests must be done with present Pulser nozzle configurations to insure that they will not erode in field applications (using current pressure drops of about $13.8 \mathrm{MPa}$ $(2,000$ psi)) at unacceptable rates?

This question is given highest priority because if acceptable life cannot be achieved the Pulser concept is compromised and the entire program must be abandoned or reoriented. This question is best answered in a test laboratory where realistic drilling fluid is used and where the cavitation number can be varied. Ideally the tests should be carried out on at least one Pulser nozzle (say $6.4 \mathrm{~mm}$ diameter) at three cavitation numbers (say $0.3,0.7,2.0$ ) with a fixed nozzle pressure drop of approximately $13.8 \mathrm{MPa}(2,000 \mathrm{psi})$. The tests should be run for an extended period of time (up to 100 hours) for each condition. The nozzle should be inspected for signs of erosion at intervals of several hours initially and longer intervals if no erosion is observed. 
Assuming that the initial nozzle is made of tungsten carbide in accordance with current practice and no significant exosion is observed, field trials using such nozzles may then be initiated. If the test nozzle shows unacceptable erosion, new nozzles using different, more erosion resistant, materials should be tested until a successful nozzle is achieved or it is concluded that erosion resistant nozzles cannot be achieved. In the event that nozzle erosion is a severe problem at a pressure drop of $13.8 \mathrm{MPa}$ $(2,000 \mathrm{psi})$, the lower pressure drop at which erosion life is acceptable should be determined. This latter information will be useful in determining if the Pulser-Fed CAVIJET might also be compromised even though the pressure drop across the Pulser element is only, say, 10 percent of the total pressure drop available for the jet.

2. What investigations are required to obtain near optimum performance of Pulser nozzles operating at a nominal pressure drop of $13.8 \mathrm{MPa}(2,000 \mathrm{psi})$ and suitable for operation in various types of mechanical bits?

A corollary question is: What investigations are required to develop a design procedure for satisfactory low Mach number Pulser nozzles?

It is assumed that both the simple Pulser and the LaidBack Pulser must be optimized. That is, it is anticipated that the simple Pulser is better than the Laid-Back Pulser when space permits but that the Laid-Back Pulser is required because of space limitations, as in a three-cone roller bit.

Three types of tests are required to develop the necessary information. These are:

- Basic flow visualization studies both inside the resonant chamber volume and within the jet over a range of cavitation 
numbers and jet speeds (near the design speed) for several changes in important features of the two design types. For example, minor changes in upstream or downstream orifice size or distance between orifices or changes in nozzle contours. Both visual observations and high speed movies of the cavitating flows should be made. This type of investigation could be carried out in the facility in Figure 23. The tests are designed to give a better understanding of the flow patterns that exist and need not necessarily be carried out fuil scale.

- Optimization tests in a test cell capable of reproducing full jet speed and cavitation numbers and preferably full scale nozzle size. The test nozzle should be instrumented so as to measure pressure fluctuations within the resonant chamber along with other flow variables. Some tests should be conducted with the jet impacting a non-erodable test plate instrumented so as to measure the fluctuating pressure at the center of the jet (and at other radial locations if possible) and some tests should be carried out using erodable rock samples. The nozzles tested would consist of families having variation in orifice shapes, sizes and spacing as well as changes to the chamber wall shapes (including baffles).

- Tests of actual bits using the best Pulser nozzles available should be carried out under full scale operating conditions. These tests should be carried out both in the laboratory (for example, at the Drilling Research Laboratory) and in the field. Field trials using the best available Pulser configurations should be started as soon as nozzles having satisfactory erosion life are available. 
3. What tests are required to develop a near optimum Pulserfed CAVIJET system?

The tests required for this system are identical to those discussed for the simple Pulser nozzle except that the program should be preceeded by further tests in air where the duct geometry between the Pulser oscillator and the CAVIJET nozzle is altered so as to reduce the energy loss in this section. Using the optimum duct geometry found, the first basic flow visualization studies should be directed toward determining whether discrete vortical eddies are formed in the jet at the critical design speed. The onset of cavitation within the Pulser chamber is also of principle interest. The test plan for optimization of the design in a high pressure test cell should be expanded to include CAVIJET nozzle speeds as high as possible while still maintaining strong pulsations.

4. What tests are required to maintain strong oscillations in a Pulser oscillator at Mach numbers higher than 0.1 ?

Air test data indicate that the Pulser oscillator response tends to fall off at Mach numbers greater than 0.1. The reason for this decline in performance is not clearly understood and has not really been studied. Presumably at the higher Mach numbers the sound wavelength approaches the chamber dimensions. Furthermore, the optimum (desired) value $\left(\forall / d_{1}{ }^{3}\right)$, the ratio of chamber volume to the cube of the orifice diameter also decreases with increasing Mach number. Thus, wave interference effects and jet-wall effects become important, and apparently in a detrimental way. If these complex factors were understood, it seems possible that the chamber shape could be designed to eliminate or reduce the interferences 
and maintain strong resonance at higher Mach numbers. There is no obvious fundamental barrier; the situation seems analogous to the difference between shallow cavity and deep cavity resonance as reported in Reference 34.

Pursuing this reasoning, higher Mach number Pulser designs should be developed and tested in air. Assuming success, the results should be used to design Pulser systems for pressures greater than $13.8 \mathrm{MPa}(2,000 \mathrm{psi})$ and the designs developed and tested in a program similar to the program outlines for the lower Mach number systems.

Although the general sequence of events in the early stages of the $R \& D$ program may be: (1) Air tests, (2) Basic flow visualization water tests, (3) High pressure test cell studies in water, (4) Bit laboratory tests under full scale conditions and (5) Field trials, a fully integrated program should involve simultaneous or near simultaneous effort in all of these activities as illustrated in Figure 54. Also shown in Figure 54 are the nozzle erosion tests required before field tests could be carried out. Figure 54 shows that each activity will raise the level of knowledge on which the design procedure for a prescribed concept (Pulser, Laid-Back Pulser, Pulser-Fed CAVIJET, other) is based and suggest new tests for further improvement. Such a process is an iterative one whose details depend on the general knowledge level or status of the design procedure at any stage of the $R \& D$ program. 


\section{REFERENCES}

1. Blueprint For A Comprehensive Federal Drilling Technology Development Program, DOE, February, 1978.

2. Maurer, W.C., "The 'Perfect-Cleaning' Theory of Rotary Drilling", Journal of Petroleum Technology, Vo1. 14, No.11 pp. $1270 \mathrm{ff}$, November 1962.

3. Maurer, W.C., Novel Drilling Techniques, Pergamon Press Oxford, 1968.

4. Proceedings, First International Symposium on Jet Cutting Technology, Coventry, England, BHRA Fluid Engineering, April 1972.

5. Proceedings, Second International Symposium on Jet Cutting Technology, Cambridge, England, BHRA Fluid Engineering, April 1974.

6. Proceedings, Third International Symposium on Jet Cutting Technology, Chicago, Illinois, BHRA Fluid Engineering, May 1976.

7. Proceedings, Fourth International Symposium on Jet Cutting Technology, Canterbury, England, BHRA Fluid Engineering, Apri1 1978.

8. Wylie, M.R.J., "Jetted Particle Drilling", 8th World Petroleum Congress, Special Paper No. 6, Moscow, Jume 1971.

9. Maurer, W.C., Heilhecker, J.K. and Love, W.W., "High-Pressure Drilling:, J. Petroleum Technology, Vo1'. 255, pp. 851-859, July, 1973, (also see Proc.; 2nd Int'1. Sympos. on Jet Cutting Technology, pp. X80-X89, April 1974).

10. Pols, A.C., "Hard-Rock Cutting", The Oil and Gas Journal, Part 1: pp. 134-144, Jan. 31, 1977, Part 2: pp. 71-75, Feb. 7, 1977.

11. Summers, D.A. et al,, "Environmental Effects on a High Pressure Jet Dril1", paper presented at the ASME Petroleum Mechanical Engineering Conference, Mexico City, Sept. 1976. 
12. Conn, A.F., and Radtke, R.P., "Cavitating Bit Jets Promise Faster Drilling for Deep-Hole Operations", The Oil and Gas Journa1, pp. 129-146, October 31, 1977 (aiso see: Trans. ASME, J. Pressure Vessel Tech., Vol. 100, pp. 52-29, 1978).

13. Leach, S.J. and Walker, G.L., "Some Aspects of Rock Cutting by High Speed Water Jets", Phil. Trans. Royal Soc., Vol. 260 pp. 295-308, 1966.

14. Conn, A.F. et a1, "Evaluation of CAVIJET ${ }^{\circledR}$ Cavitating Jets for Deep-Hole Rock Cutting", SAND81-7067, May 1981.

15. Conn, A.F., and Rudy, S.L., "A Cavitating Water Jet for Fouling Removal", Proceedings, Fourth International Congress on Marine Corrosion and Fouling, Juan-Les-Pins, France, pp. 97-103, June 1976.

16. Conn, A.F . "App1ication of a CAVIJET ${ }^{\circledR}$ System for Removing Marine Fouling and Rust", Proceedings, 19 th American Towing Tank Conference, Vo1. Two, pp. 415-428, August, 1977.

17. Conn, A.F., and Rudy, S.L., "Cutting Coal with the CAVIJET Cavitating Water Jet Method", Paper D8 in Ref. 5, Ma.y, 1976.

18. Conn, A.F. and Rudy, S.L., "Parametric Study of Coal Cutting with the CAVIJET@ Cavitating Water Jet Method", HYDRONAUTICS, Incorporated Technical Report 7511-1, September 1976 (NTIS No. PB 266-060/AS).

19. Conn, A.F. and Rudy, S.L., "Using Cavitating Water Jets for Demilitarization", Proceedings, Symposium on Demilitarization of Conventional Munitions, ADPA, April, 1976.

20. Conn, A.F., Krajkowski, E.A. and Shapira, N.I., "Using the CAVIJET@ Process to Remove Explosives from Army Munitions", Proceedings, Second Demilitarization and Disposal Technology Conference, ADPA, pp. 38-40, April, 1979.

21. Johnson, V.E., Jr. et al, "Tunnelling, Fracturing, Drilling and Mining with High Speed Water Jets Utilizing Cavitation Damage", Paper A3 in Reference 3, Apri1, 1972.

22. Crow, S.C. and Champagne, F.H., "Orderly Structure in Jet Turbulence", Journal of Fluid Mechanics, Vol. 48, Part 3, August 1971. 
23. Conn, A.F., et al. "Evaluation of CAVIJET" Cavitating Jets For Cutting Coal-Associated Rocks " HYDRONAUTICS, Incorporated Technical Report 7774-1, April 1980.

24. Maurer, W.C., "Advanced Drilling Techniques," Maurer Engineering Incorporated Technical Report 79-1, January 2, 1979.

25. Proceedings, Fifth International Symposium on Jet Cutting Technology, Hanover, Germany, BHRA Fluid Engineering, June 1980 .

26. Wylie, E.B., "Pipeline Dynamics and the Pulsed Jet," Paper A5 in Reference 4, April, 1972.

27. Streeter, V.I and Wylie, E.B., Hydraulic Transients, McGraw Hill, 1967.

28. Nebecker, E.B. and Rodriguez, S.E., "Percussive Water Jets for Rapid Excavation," Scientific Associates Report (NTIS-772931), 1973.

29. Galle, E.M. and Woods, H.B., United States Patent Number $3,405,770$, October 15, 1968 .

30. Fü1öp, M. and R6kar, G., United States Patent Number 4,071,097, January 31, 1978.

3I. Sondhauss, C., Annual Physics, Volume 97, p. 1854.

32. Helmholtz, Philosophical Magazine, Volume 36, p. 337 , 1868 .

33. Rayleigh, J.W.S., Theory of Sound, Chapter XXI, 2nd Edition, p. 1826 ff, MacMillan (Republished 1945, Dover Publications).

34. Rockwe11, D. and Naudascher, E., "Se1f Süstained Oscillations of Impinging Free Shear Layers", Annual Review of Fluid Mechanics, Volume 11, 1979.

35. Leconte, J., Philosophical Magazine, Volume 15, 1858 . 
36. Moore, C.J., "The Role of Shear Layer Instability Waves In Jet Exhaust Noise", Journal of Fluid Mechanics, Volume 80 , pp. $321-367,1977$.

37. Kibens, V., "Discrete Noise Spectrum Generated By An Acousticaliy Excited Jet", Paper 79-0592, AIAA Fith Aeroacoustics Conference, March 12-14, 1979.

38. Morel, T., "Experimental Study of a Jet Driven Helmholtz Osciliator", ASME Journal of Fluids Engineering, Vo1. 101, pp. 383-390, September, 1979 . 

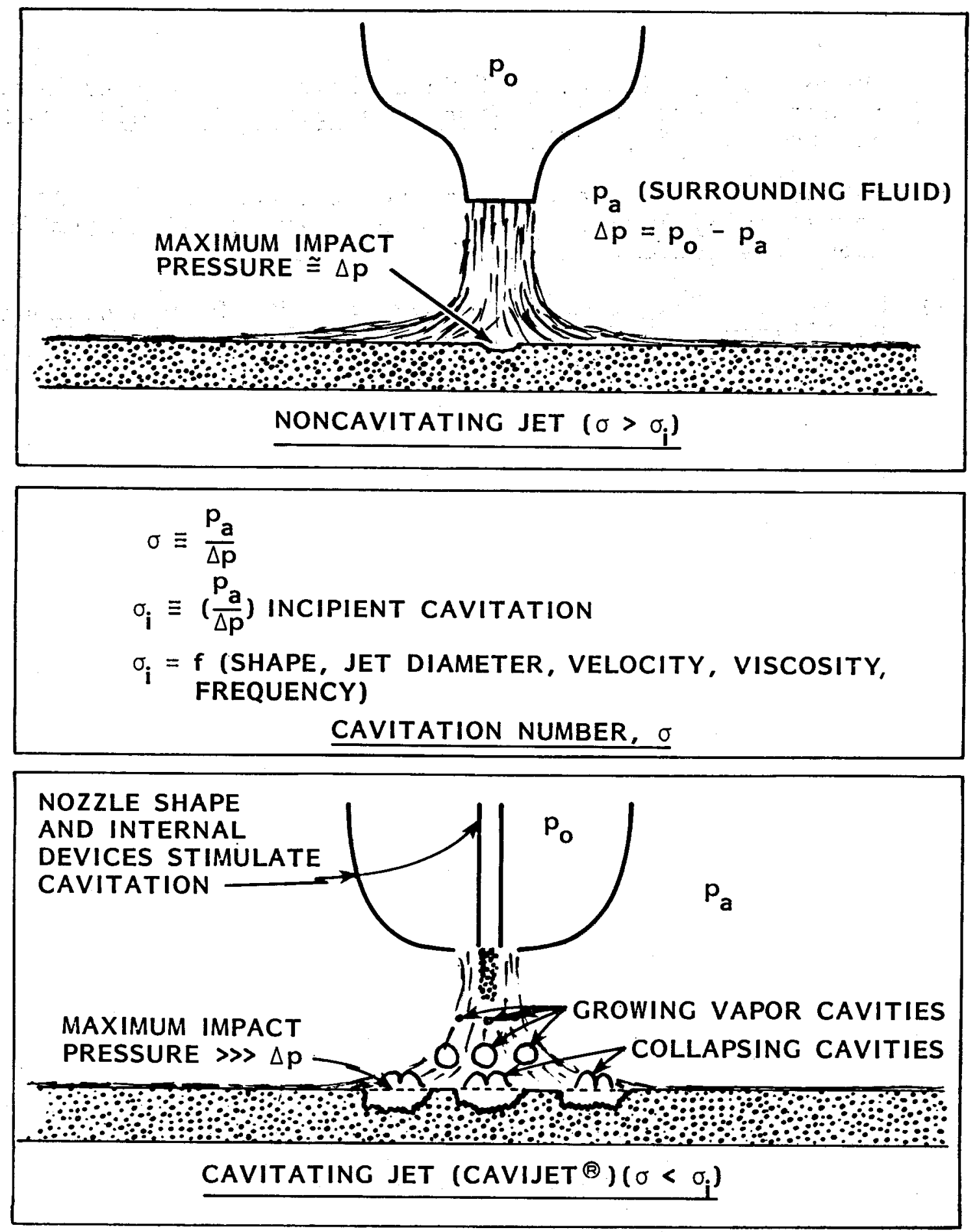

FIGURE 1 - COMPARISON OF CAVITATING AND NON-CAVITATING JETS 


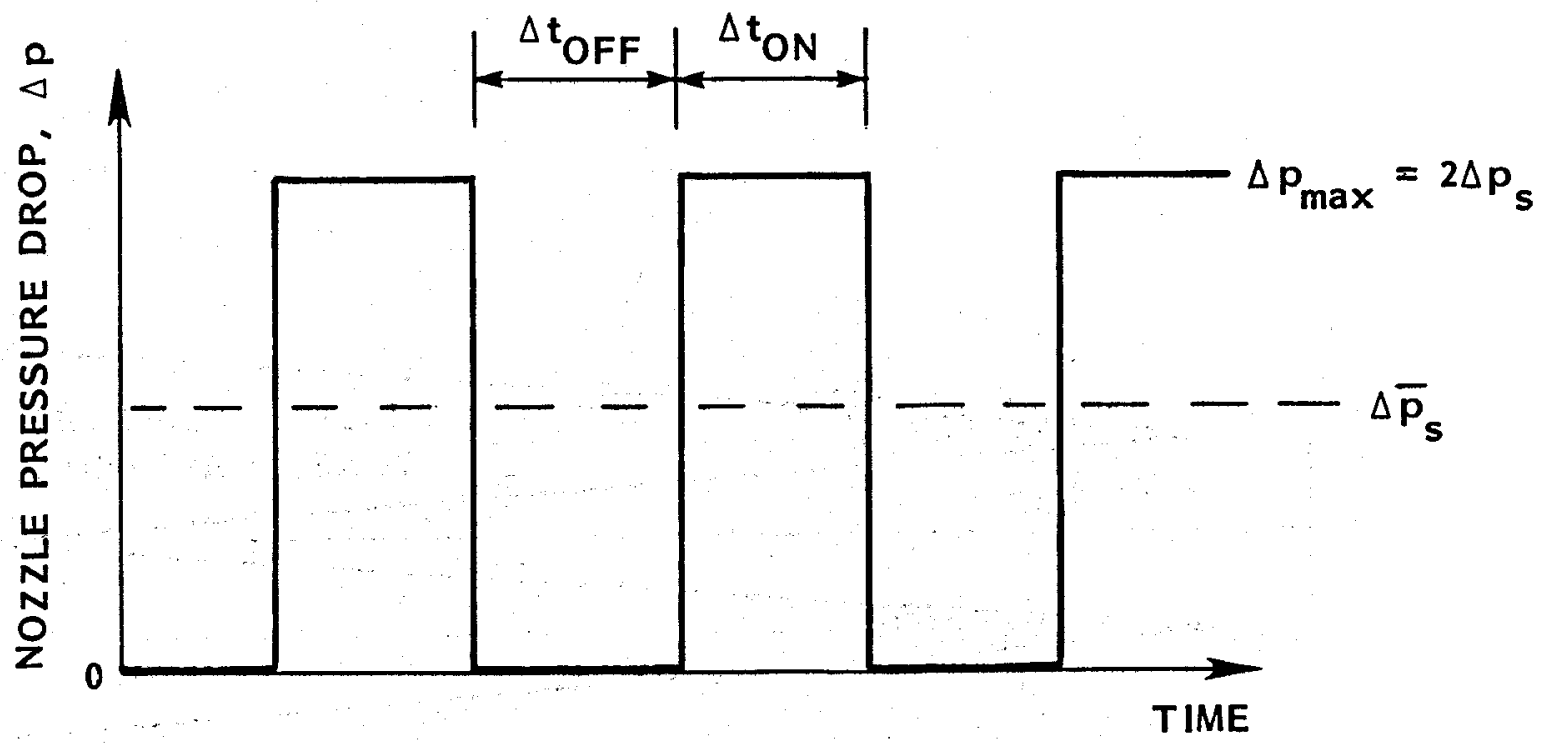

a. SQUARE WAVE MODULATION

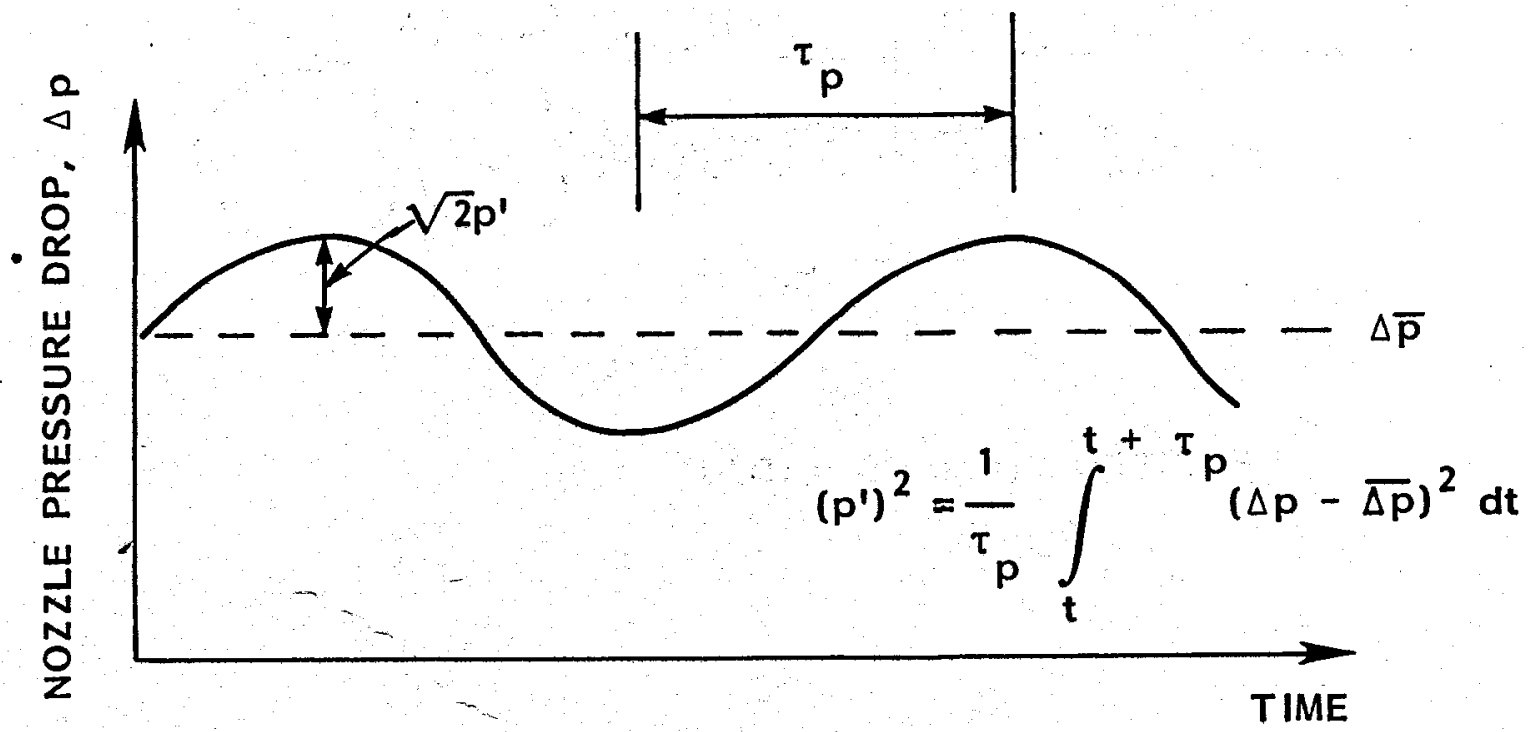

b. SINE WAVE MODULATION

FIGURE 2 - PULSING CONCEPT DEFINITIONS 

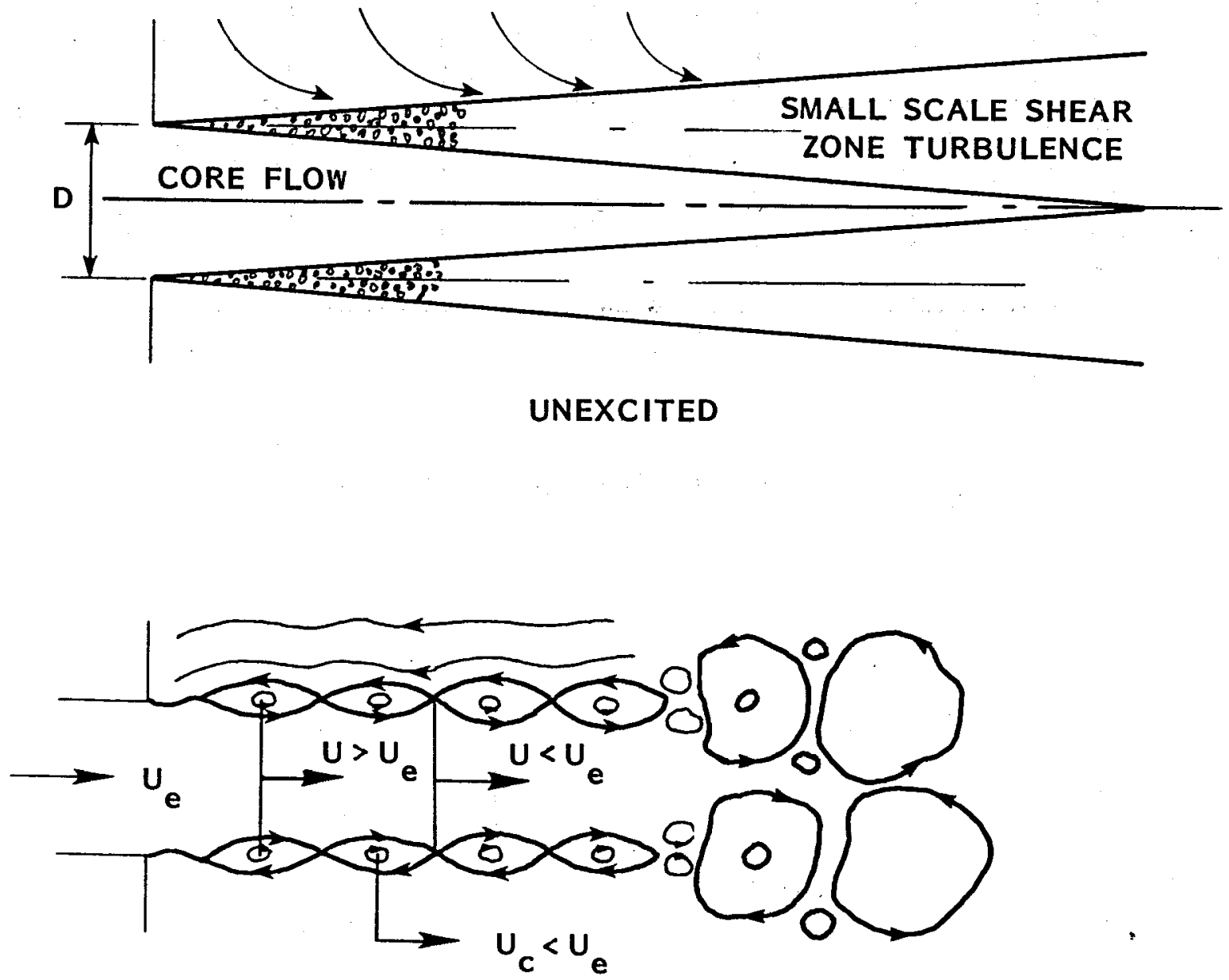

EXCITED $(0.2 \approx S=f D / U \approx 1.0)$

FIGURE 3 - COMPARISON OF FLOW PATTERNS FOR EXCITED AND UNEXCITED SUBMERGED JETS 


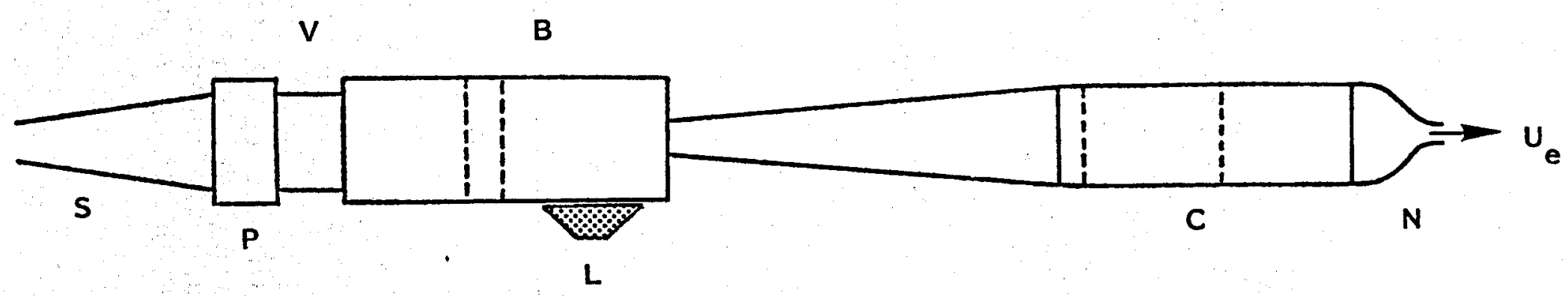

FIGURE 4 - SCHEMATIC OF THE JET FACILITY USED IN REFERENCE 22 TESTS 


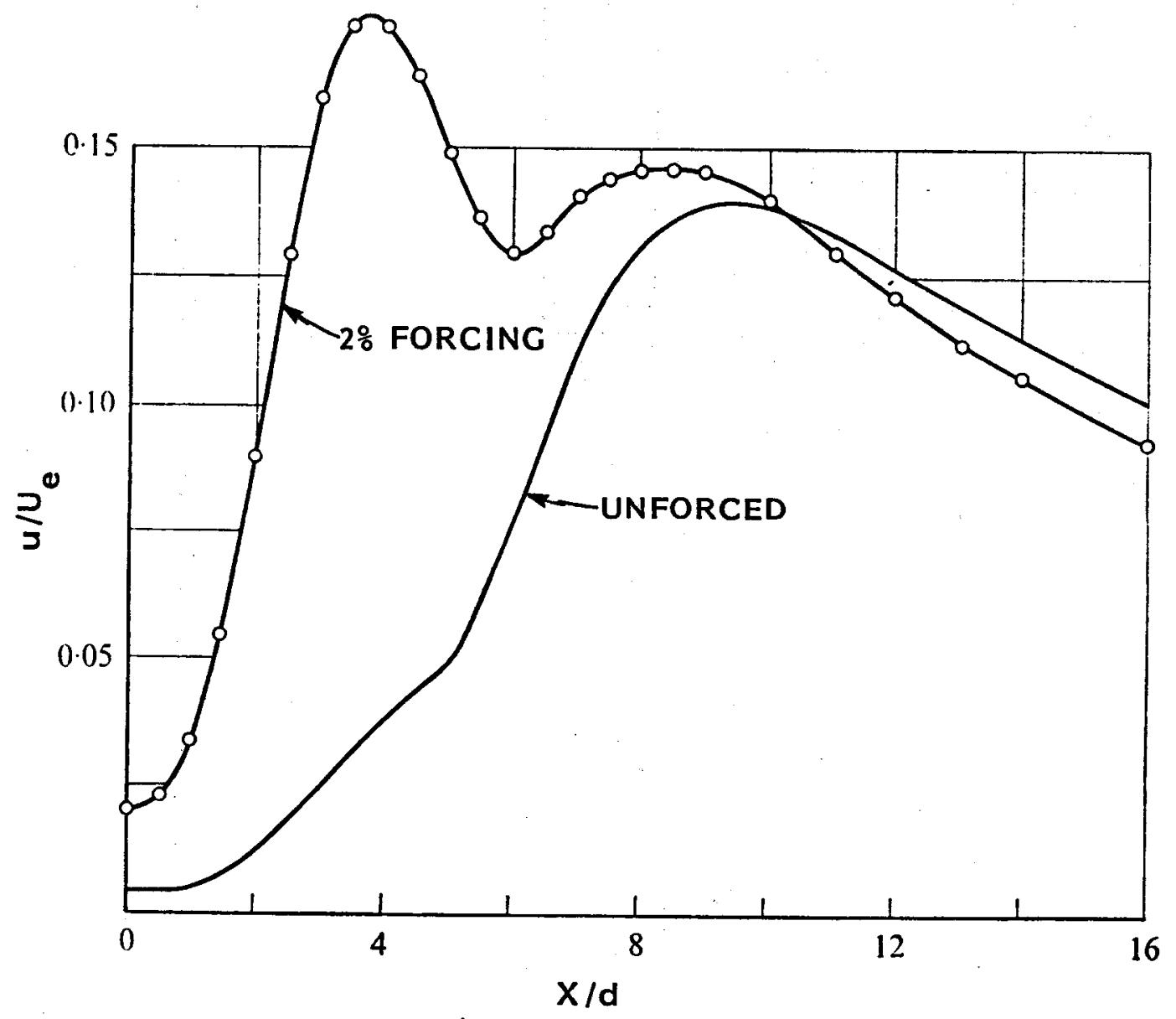

FIGURE 5 - AXIAL PROFILE OF THE TURBULENCE INTENSITY, MEASURED ALONG THE CENTERLINE UNDER $2 \%$ FORCING AT A STROUHAL NUMBER OF $\mathbf{0} \cdot \mathbf{3 0}$. THE CURVE WITHOUT DATA POINTS REPRESENTS THE UNFORCED CASE (AFTER REFERENCE 22) 


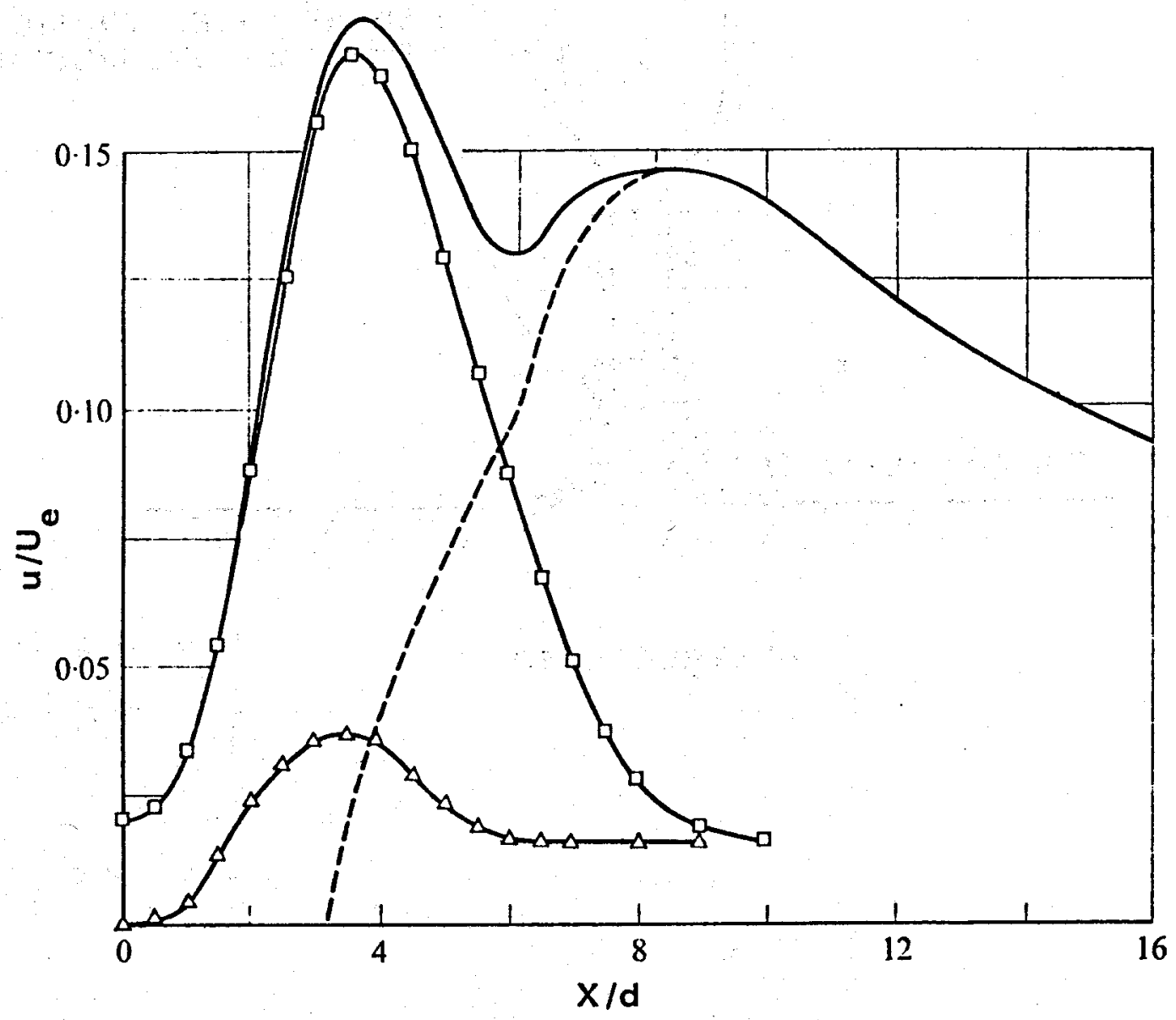

Filtered axial profiles of the preferred mode. The solid curve without data points represents the total intensity $u / U_{e}$ and is taken from figure 5. The square data symbols denote the contribution $u_{0.30} / U_{e}$ of the fundamental, and the triangular symbols denote the contribution $u_{0 \cdot 60} / U_{e}$ of the harmonic. The dashed curve represents the intensity of turbulence not bound in the fundamental or harmonic.

FIGURE 6 - COMPONENTS OF THE STRUCTURAL MODULATION IN A JET EXCITED AT $S^{*}{ }_{d}=0.30$ (AFTER REF. 22) 


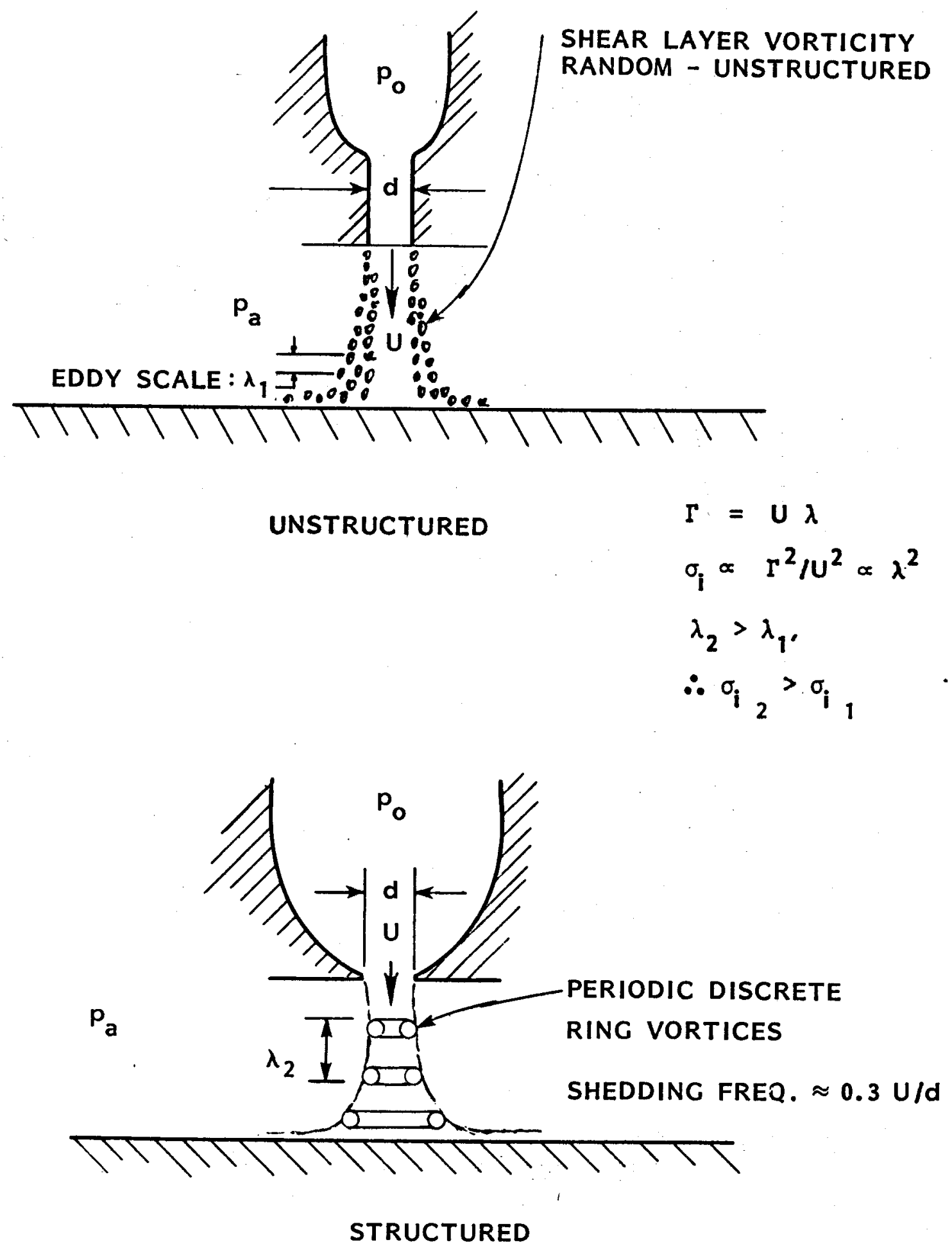

FIGURE 7 - COMPARISON OF SHEAR ZONE CAVITATION IN SUBMERGED STRUCTURED AND UNSTRUCTURED JETS 


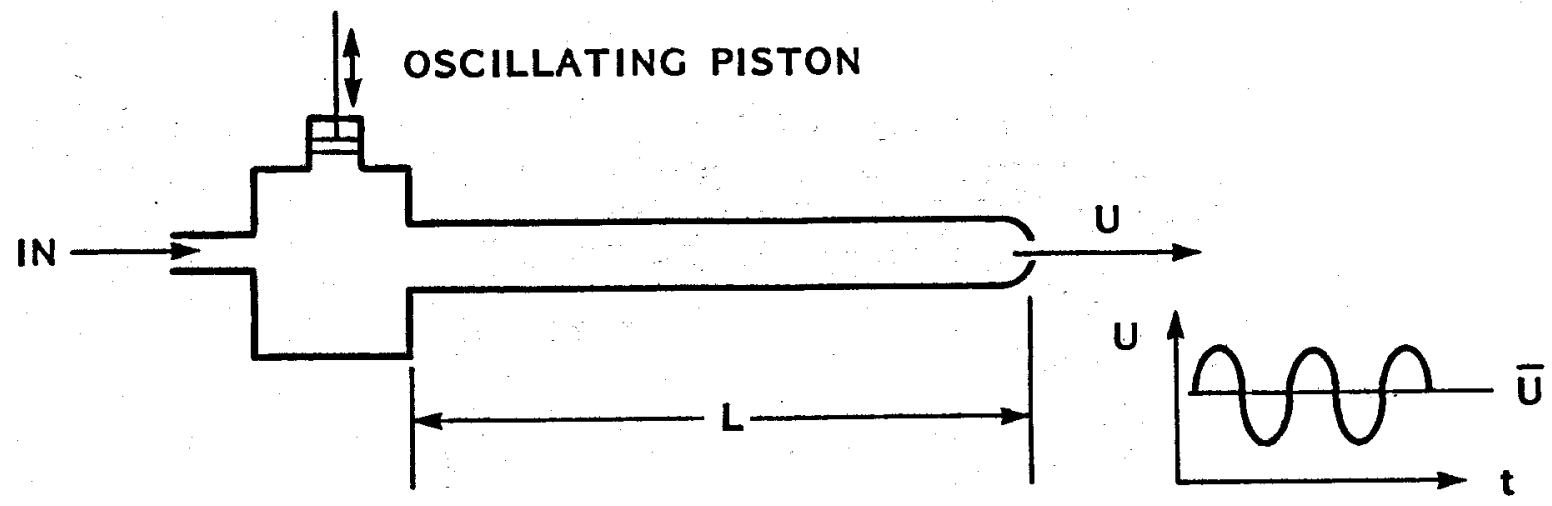

a. OSCILLATING PISTON

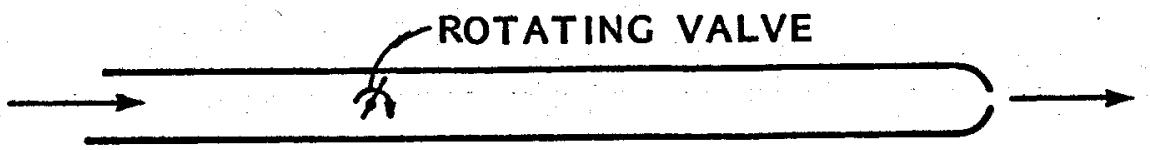

b. OSCILLATING MECHANICAL VALVE

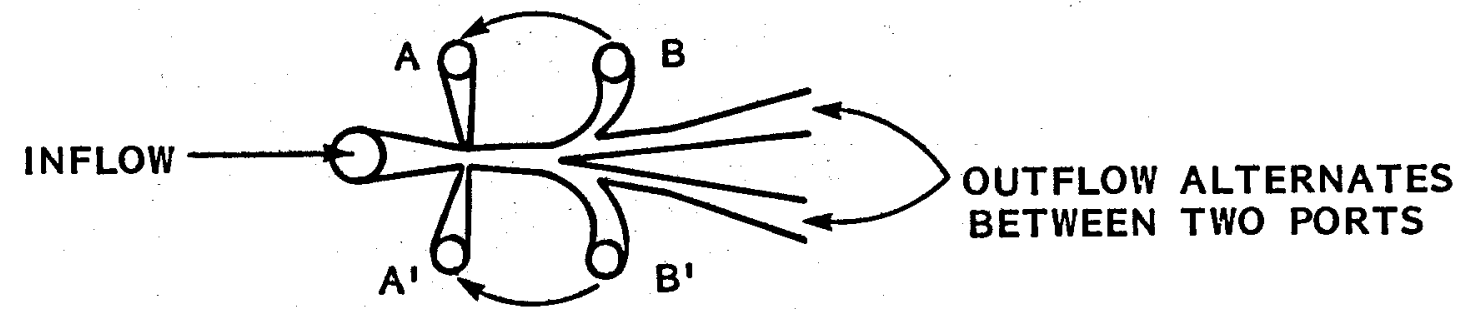

c. FLUID OSCILLATOR VALVE

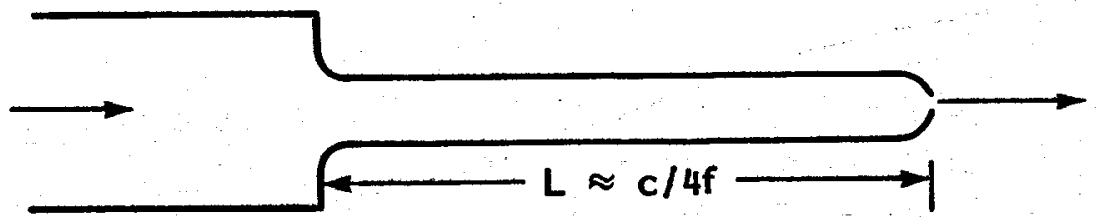

d. ORGAN-PIPE ACOUSTIC OSCILLATOR

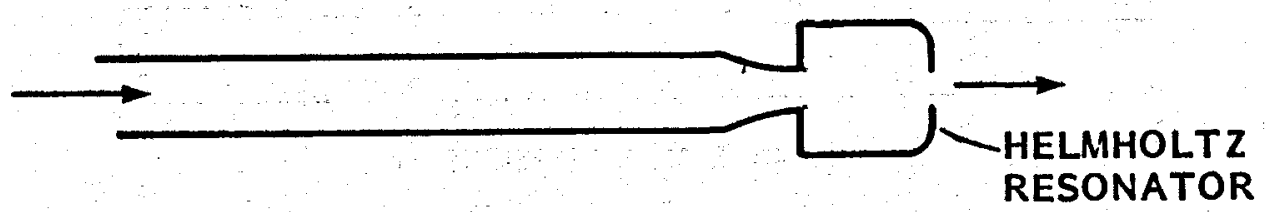

e. HELMHOLTZ ACOUSTIC OSCILLATOR

FIGURE 8 - GENERAL CONCEPTS FOR PULSING JETS 

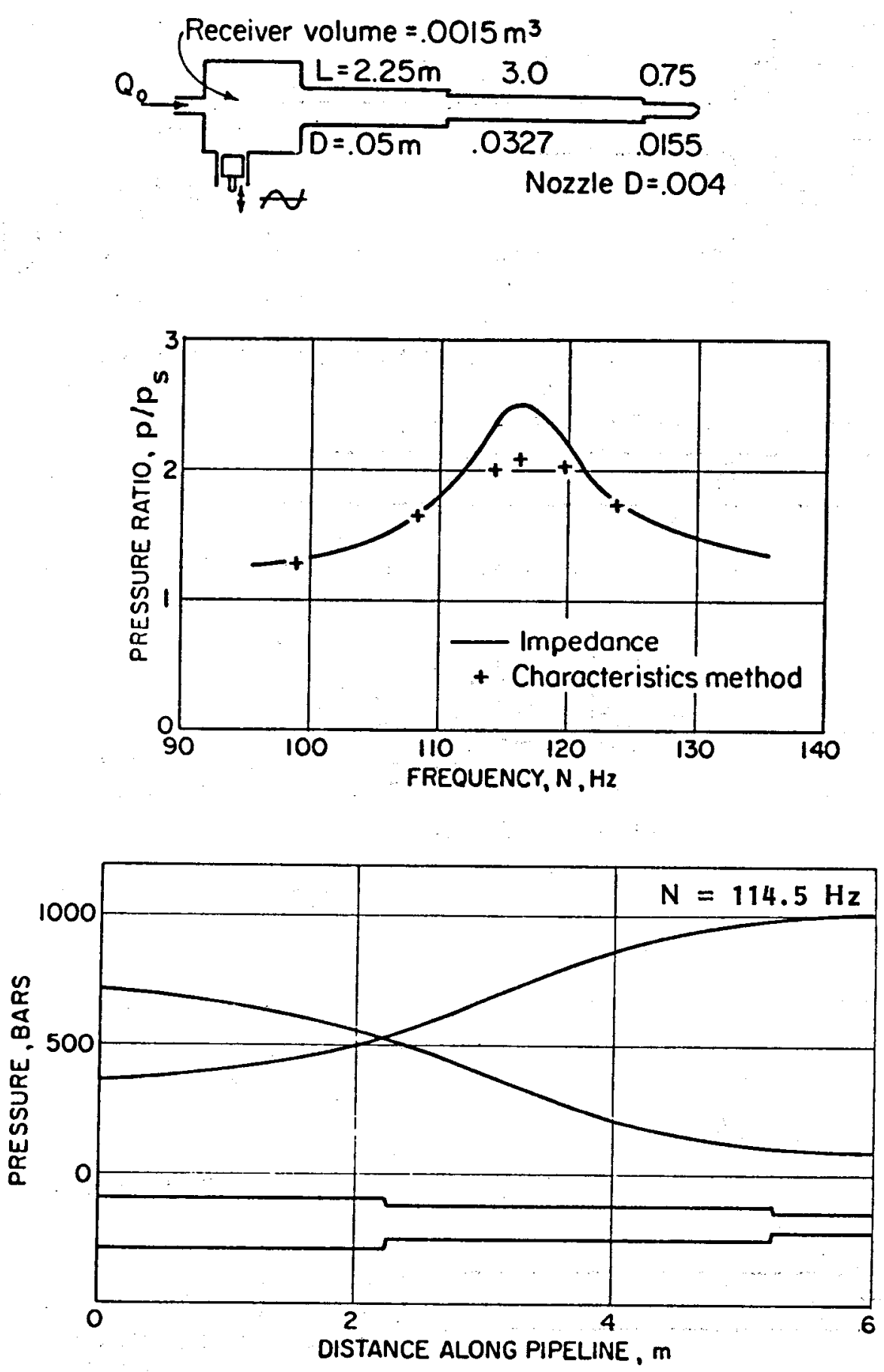

FIGURE 9 - THEORETICAL PERFORMANCE OF A MECHANICALLY FORCED, RESONANT PULSING SYSTEM (AFTER REF. 26) 


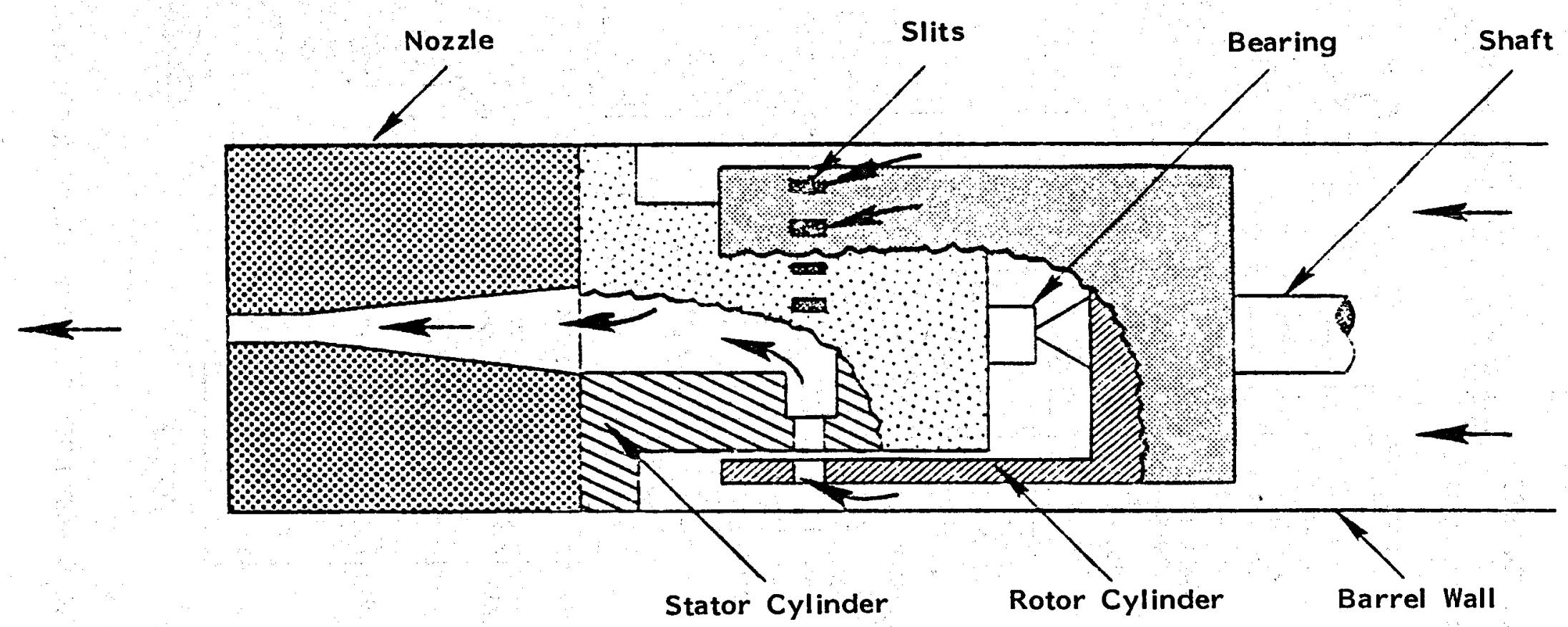

FIGURE 10 - FLOW RATE MODULATOR ( AFTER REF. 28) 
Oct. 15, 1968

E M. GALLE ETAL

DRILLTEO YETHOD AFD APPARATUS EMPLOTIKG

$3,405,770$

F11ed Iay 25, 1966

7 Sheets-Sheet

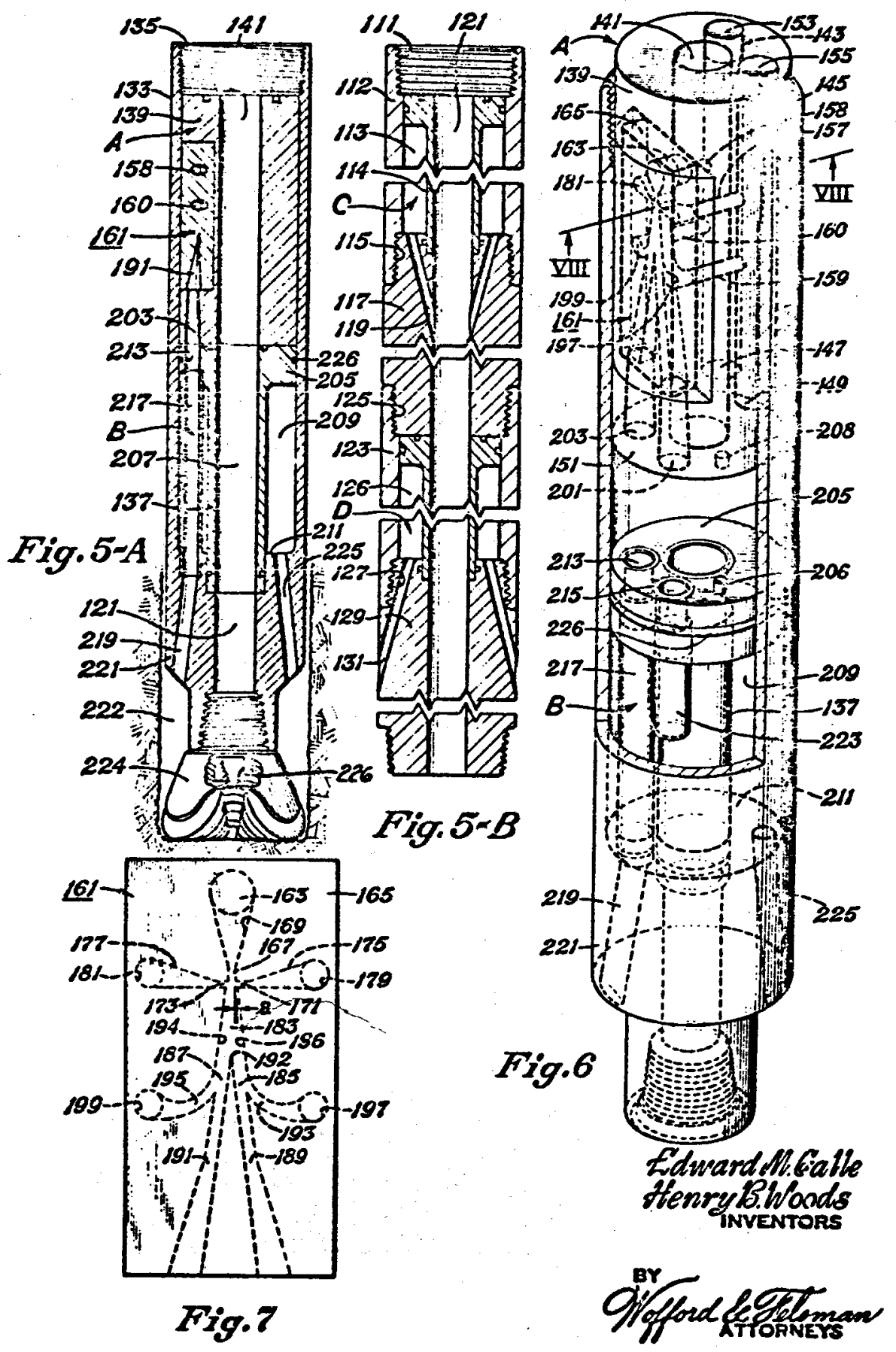

FIGURE 11 - FLUID AMPLIFIER PULSING DEVICE SUGGESTED IN REF. 29 


\section{U.S. Patent Jan. 31, $1978 \quad$ Sheet 1 of $2 \quad 4,071,097$}

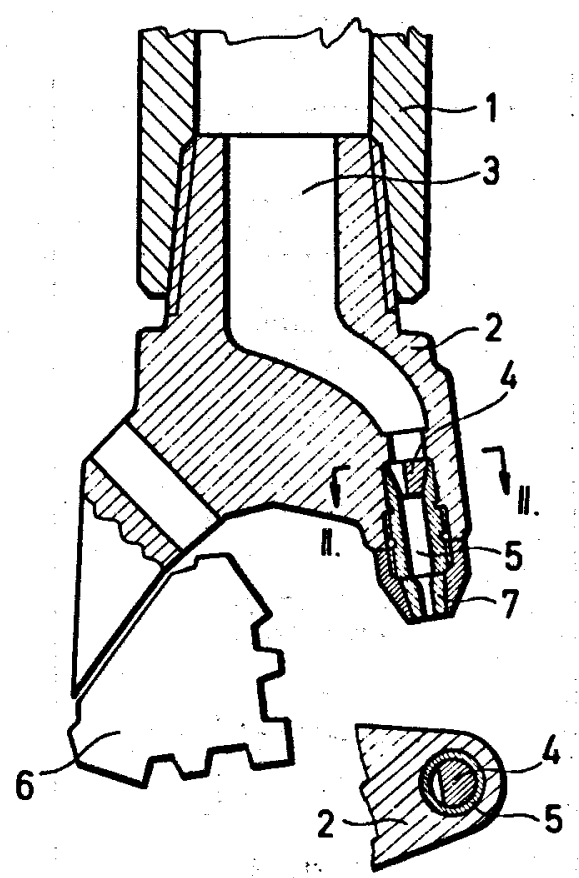

Fig.1

Fig.2

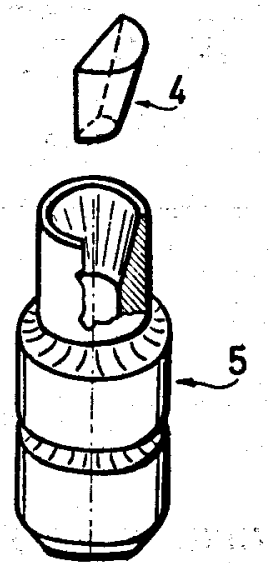

Fig.3

FICURE 12 - FÜLÖPIS ULTRASONIC "FLUID WHISTLE" OSCILLATOR CONCEPT (AFTER REF. 30) 


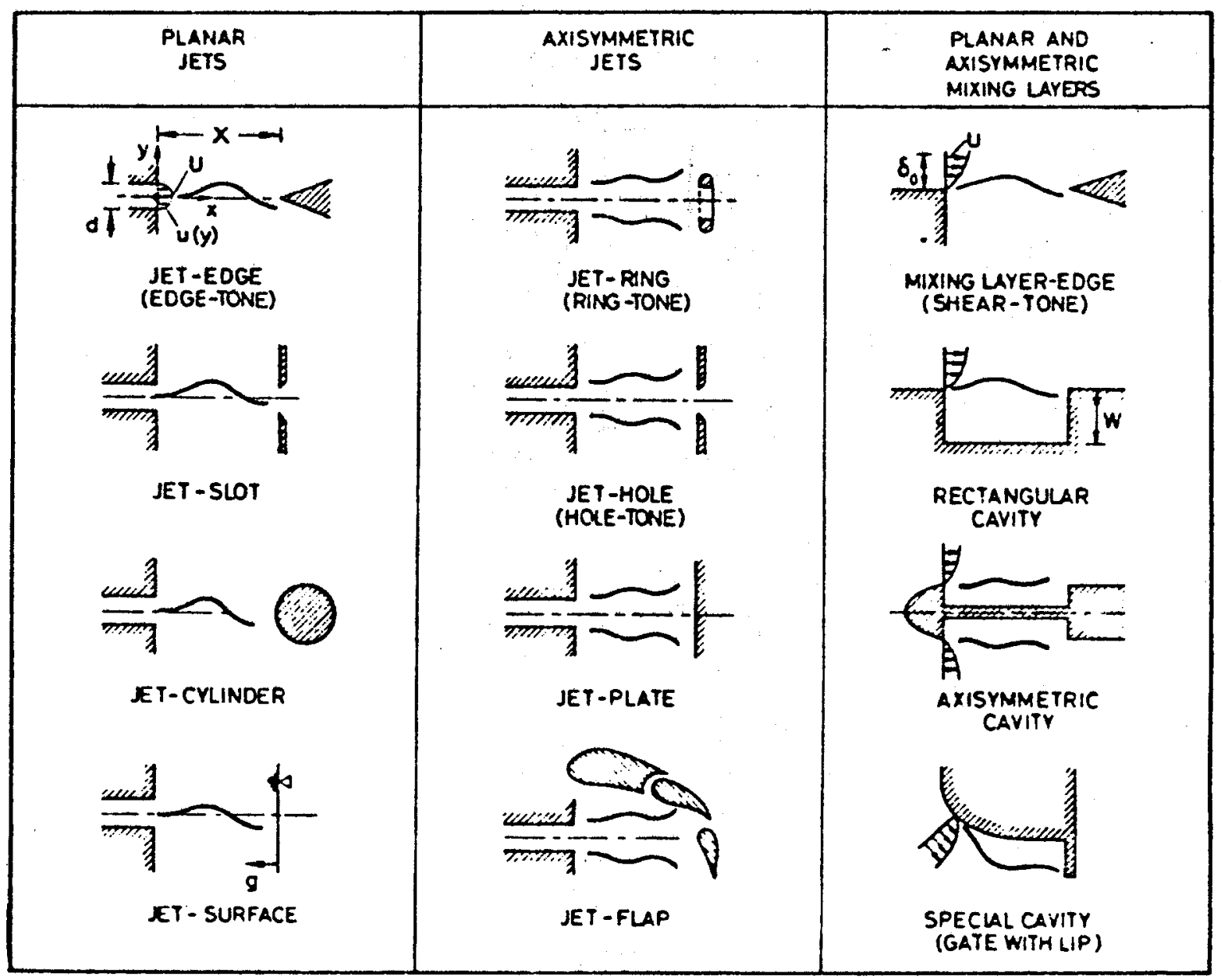

FIGURE 13 - EXAMPLES OF GEOMETRIES THAT CAUSE SELF-SUSTAINED OSCILLATION IN SHEAR FLOWS (AFTER REF. 34) 


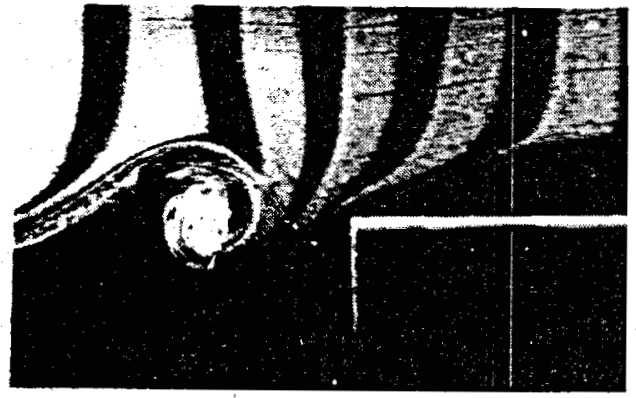

(a)

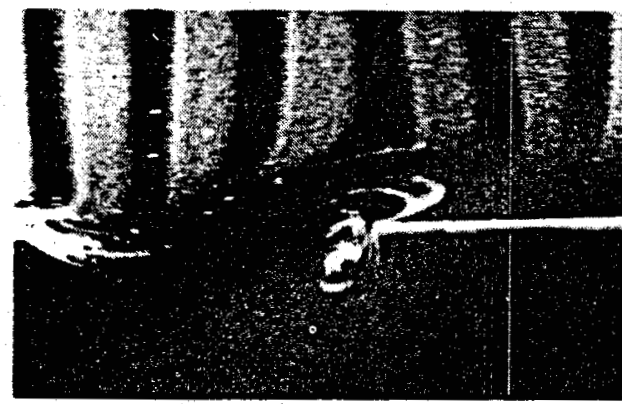

(c)

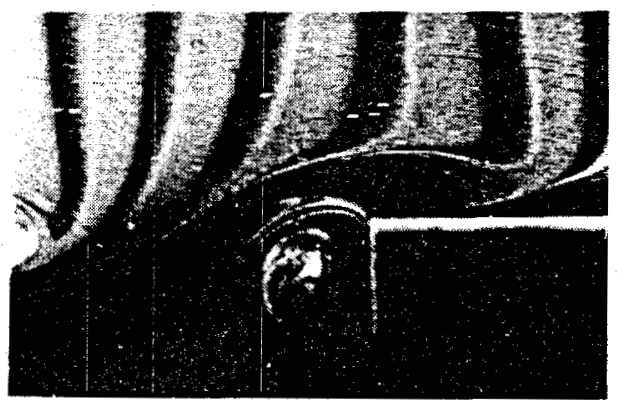

(b)

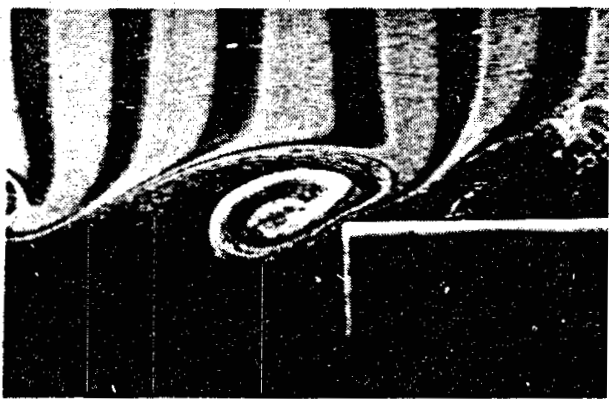

(d)

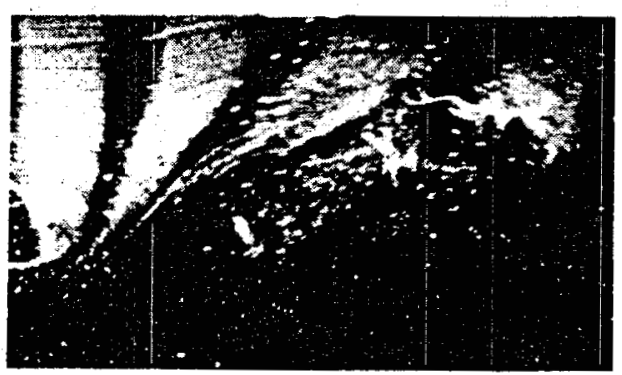

(e)

Hydrogen bubble visualization of: (a) typical vortex approaching impingement edge of a cavity; (b) complete clipping of vortex; (c) partial clipping of vortex; and (d) escape of vortex. Also shown is same flow without an edge $(e)$. $\operatorname{Re}_{\theta_{0}}=106, L / \theta_{0}=142$ (Rockwell \& Knisely 1978).

FIGURE 14 - FLOW VISUALIZATION OF VORTEX IMPINGEMENT (AFTER REF. 34) 


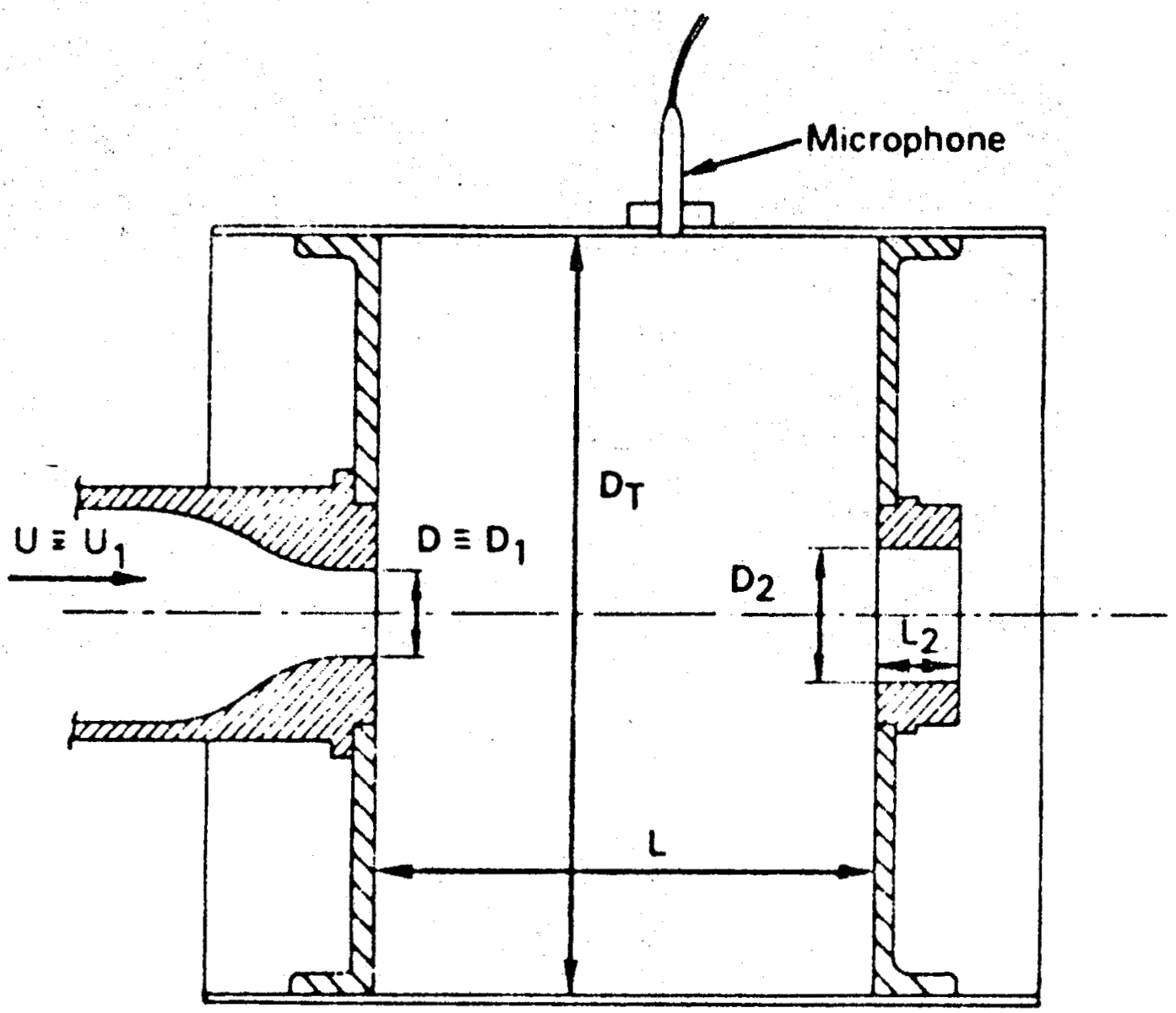

FIGURE 15 - EXPERIMENTAL SET-UP FOR JET-DRIVEN HELMHOLTZ OSCILLATORS (AFTER REF. 38) 


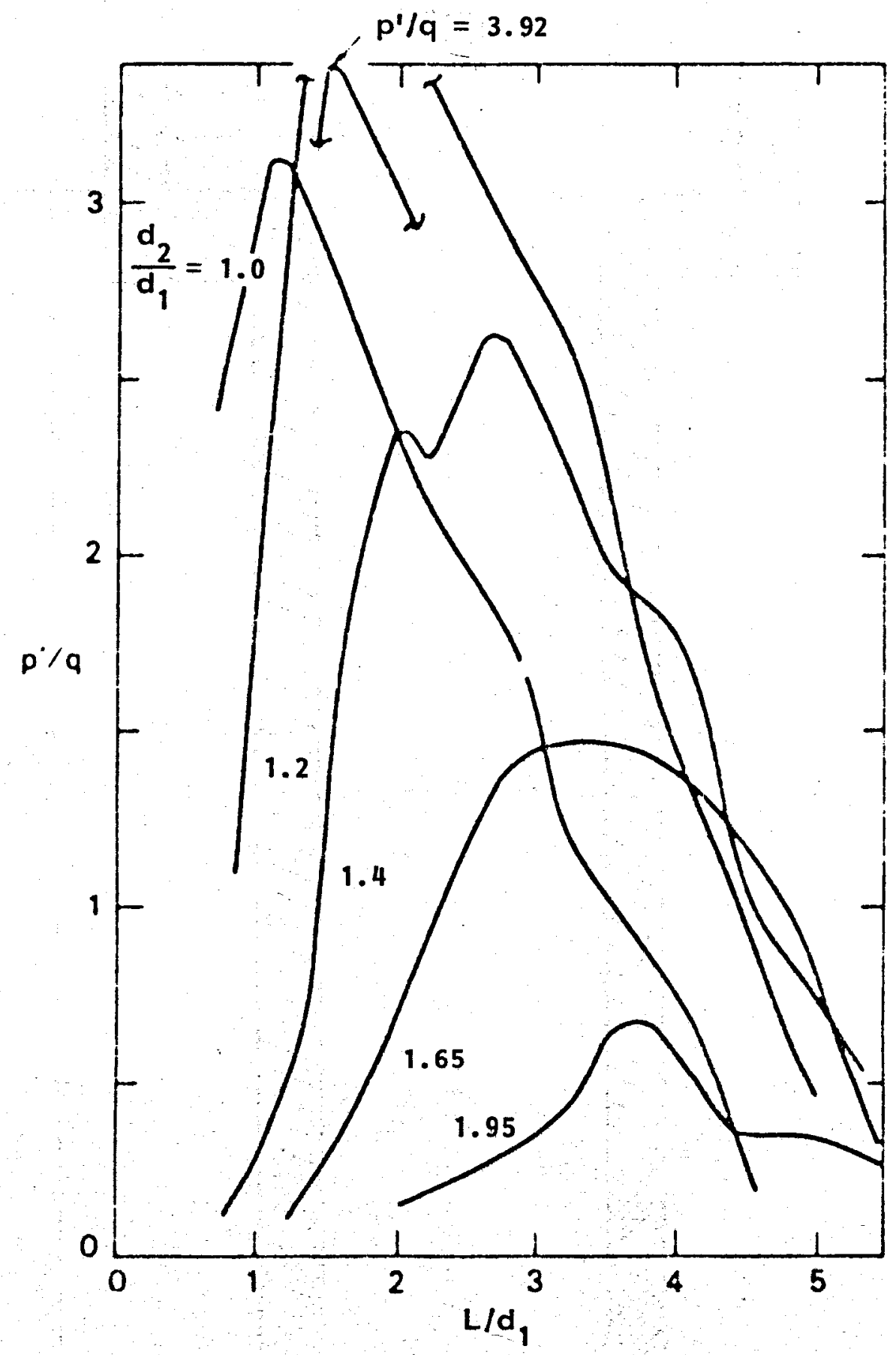

FIGURE 16 - MEASURED PEAK RIIS CHAMBER PRESSURE

FLUCTUATIONS IN MODE 2 ( AFTER REF. 38) 


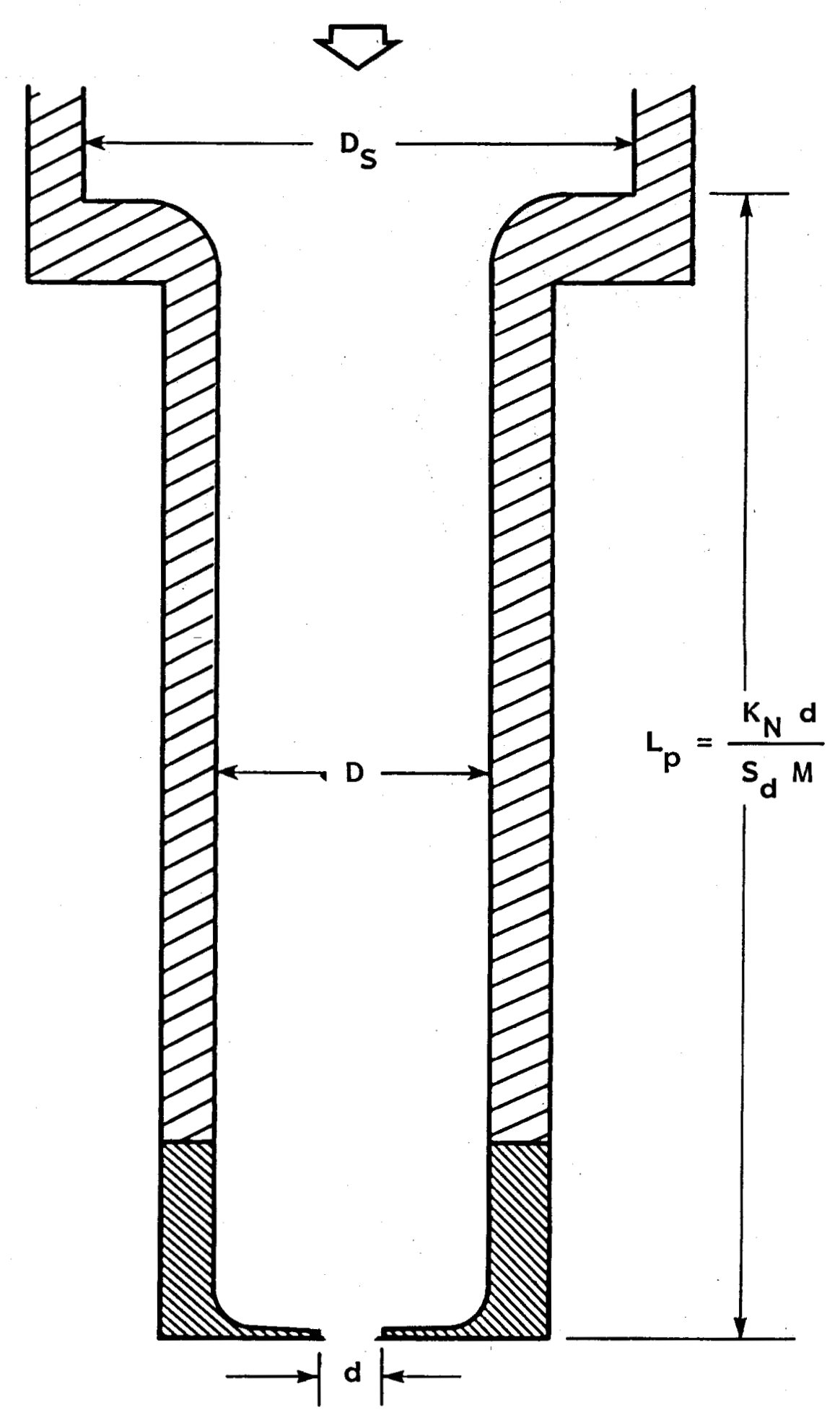

FIGURE 17 - ORGAN-PIPE RESONANT CAVITY CONCEPT

90 


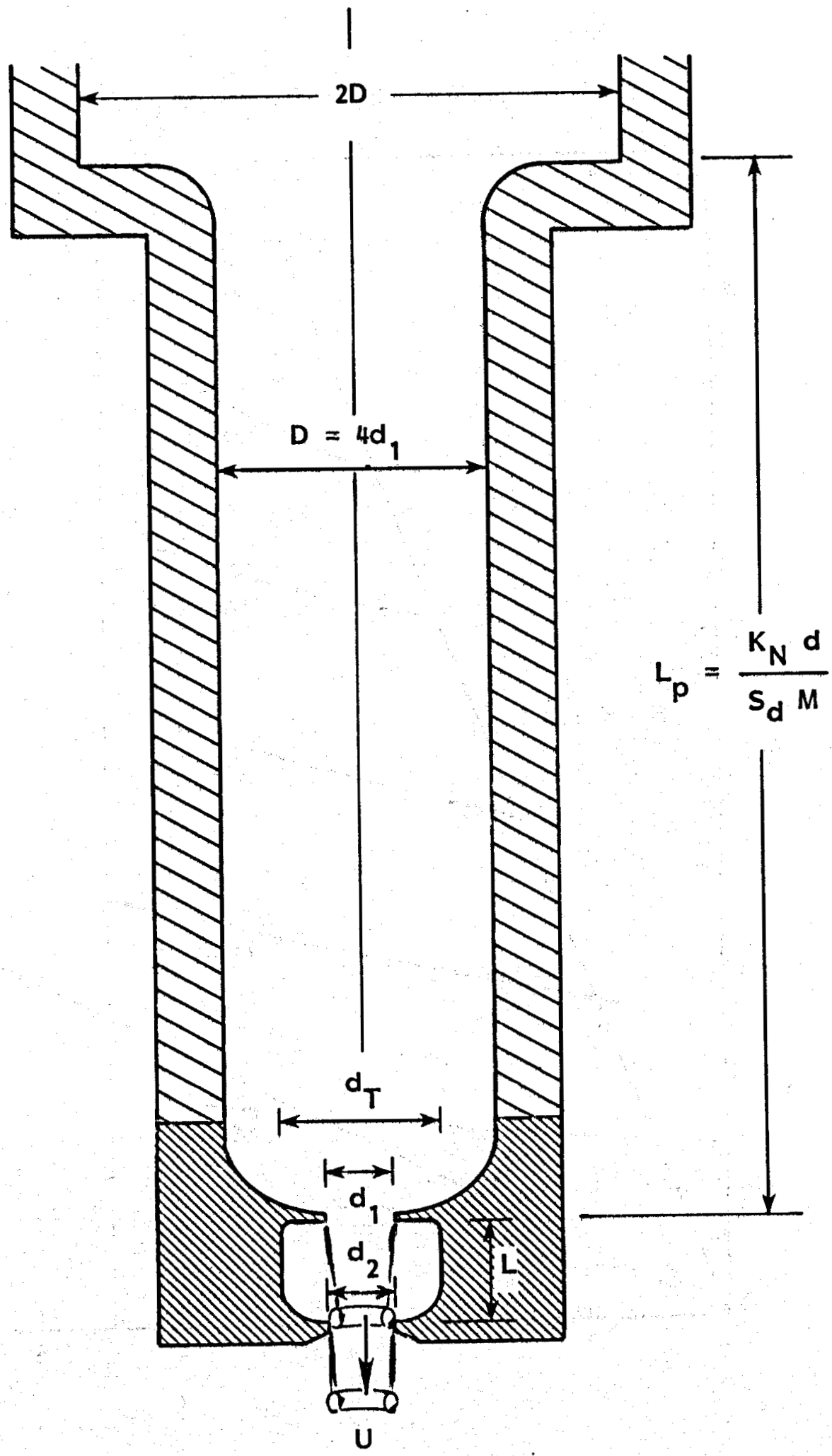

FIGURE 18 - "PULSER" OR TANDEM-ORIFICE, HELMHOLTZ RESONATOR CONCEPT (WITH ORGAN PIPE FEED) 


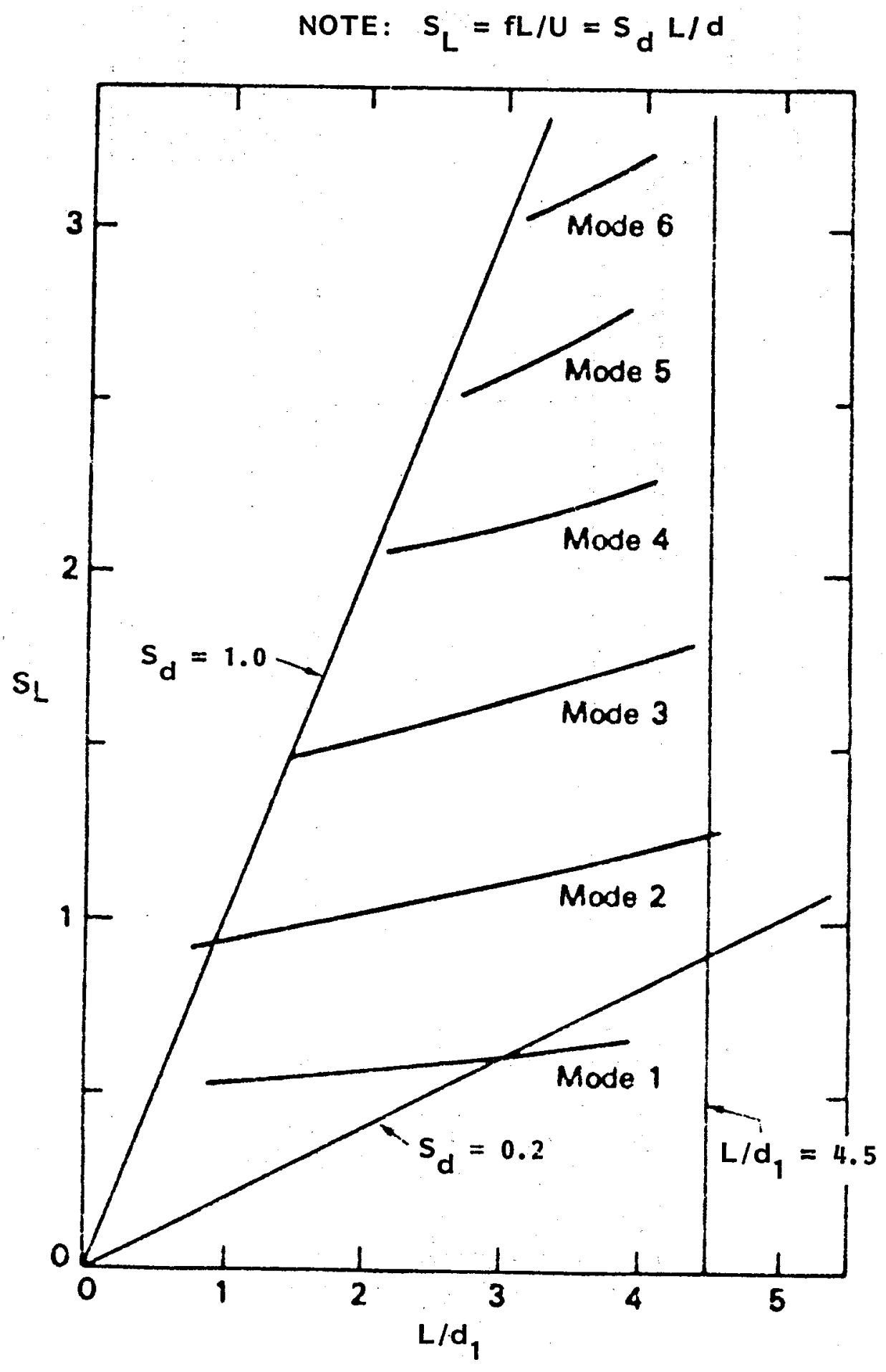

FIGURE 19 - CONDITIONS FOR STRONG JET DRIVEN HELMHOLTZ OSCILLATOR (PULSER) OPERATION (AFTER REF. 38) 

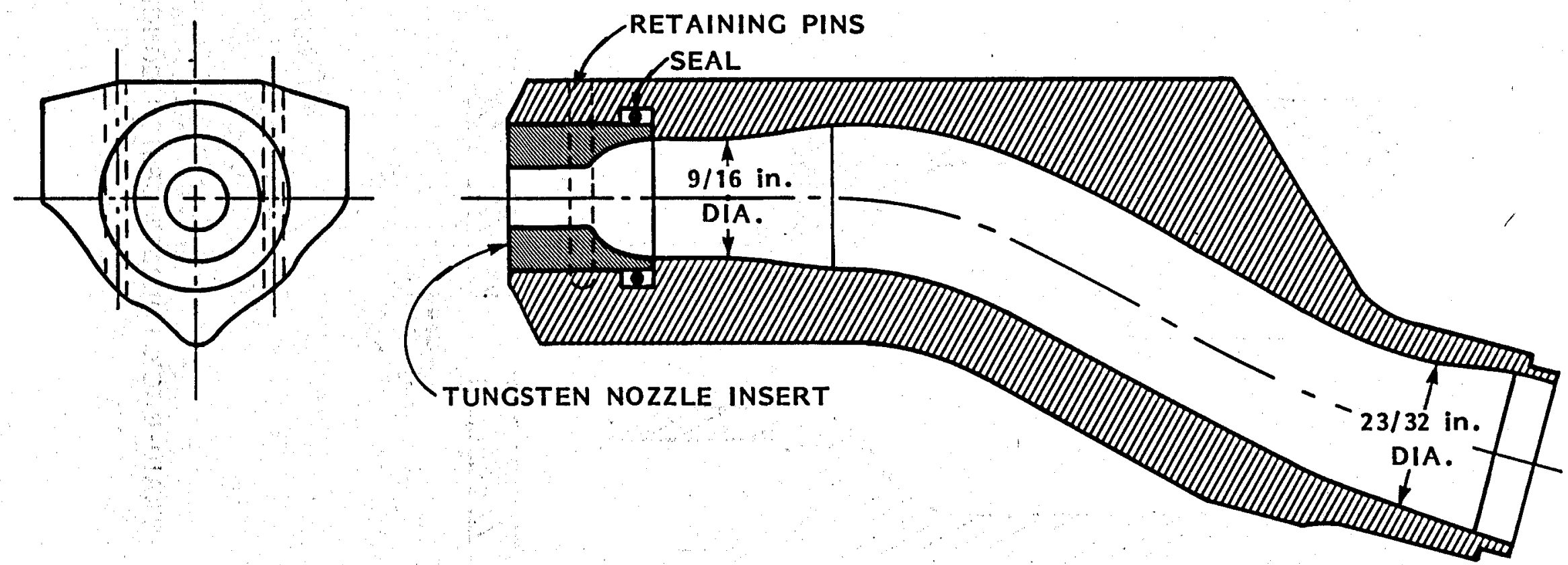

FIGURE 20 - EXTENDED JET NOZZLE CASTING AND INSERT FOR A TYPICAL 9-7/8 INCH, 3-CONE ROLLER BIT 


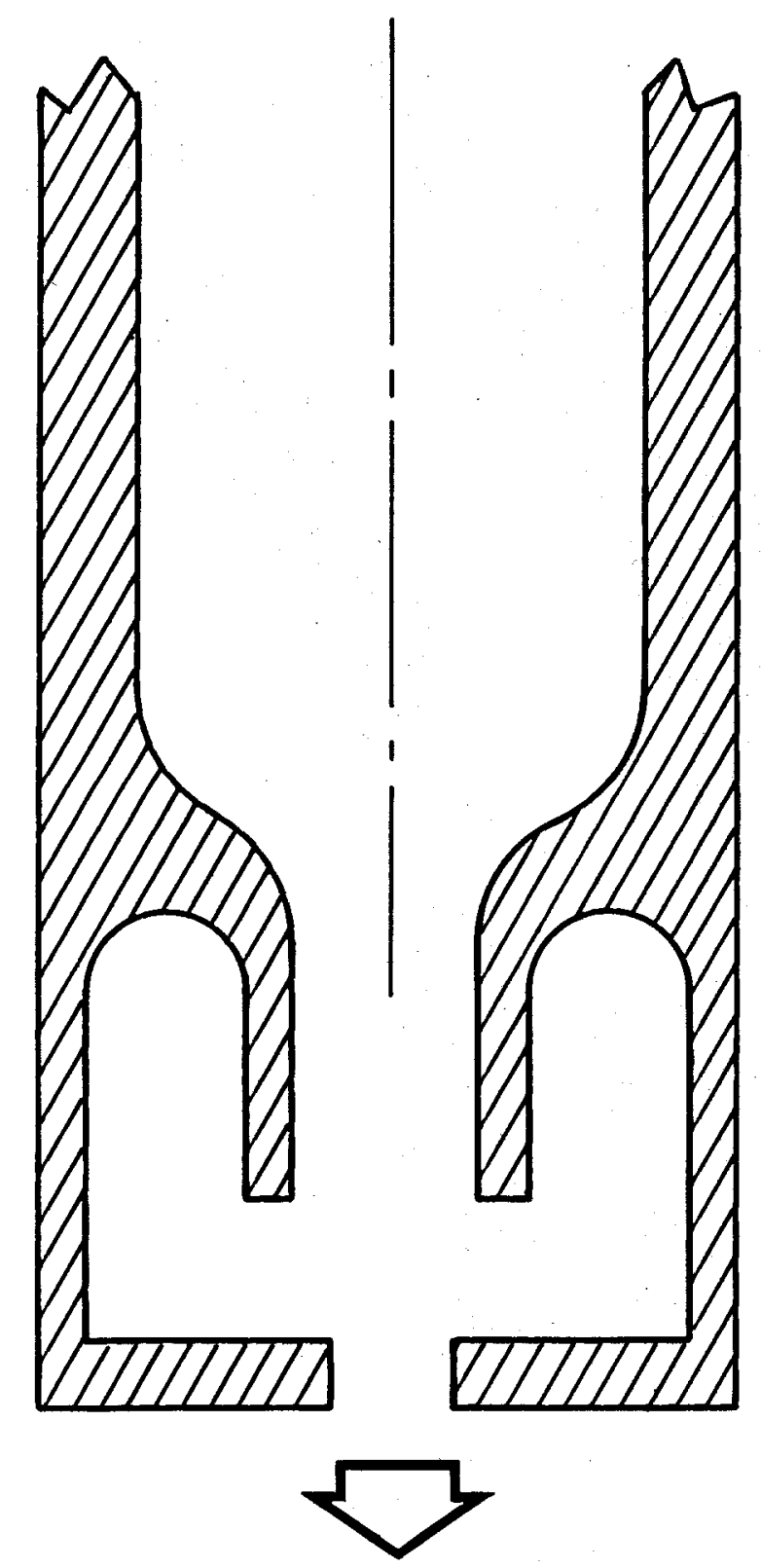

FIGURE 21 - "LAID-BACK PULSER" DESIGN CONCEPT 


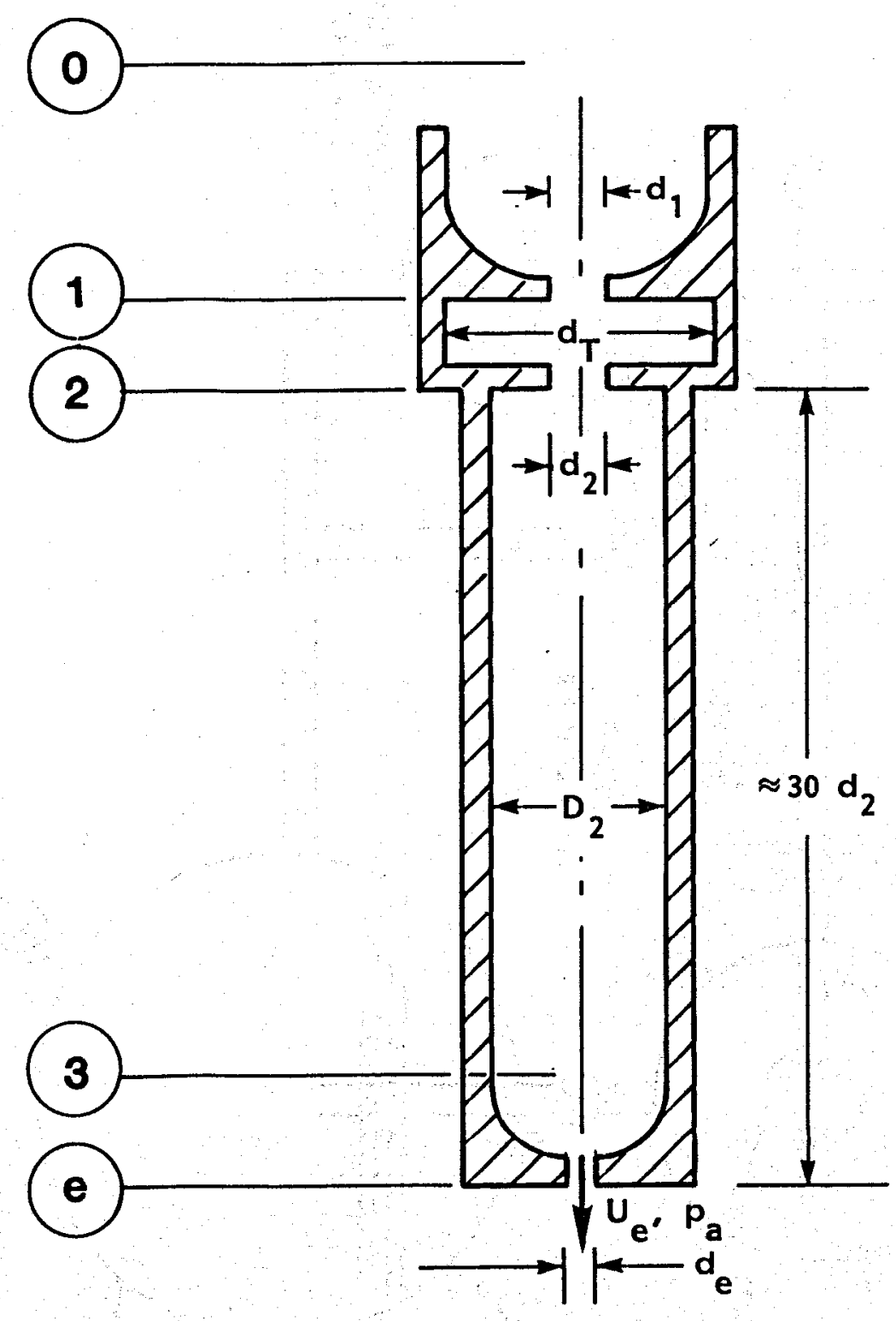

FIGURE 22 - "PULSER-FED" CAVIJET CONCEPT 


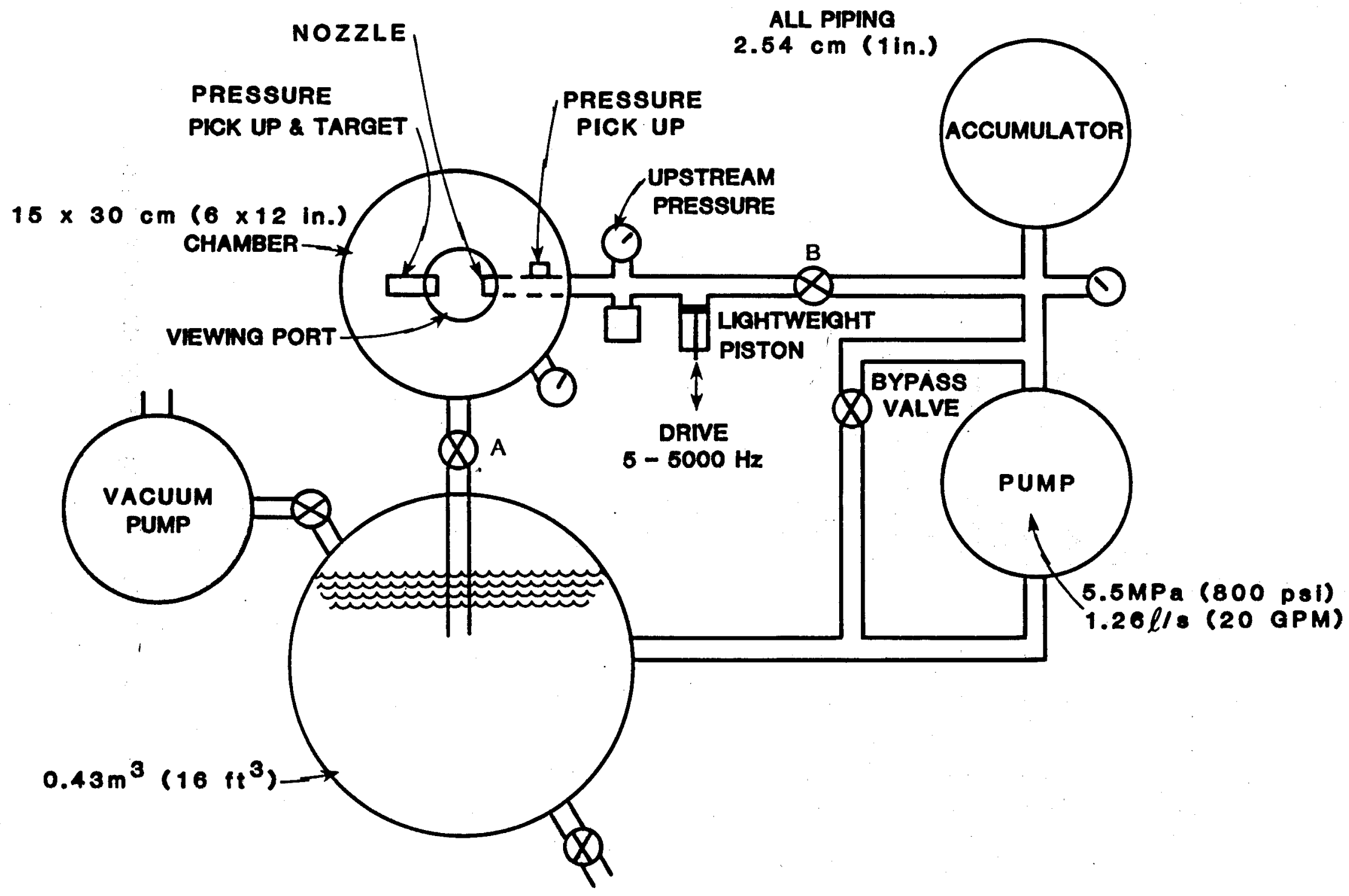

FIGURE 23 - TEST APPARATUS FOR STUDYING PULSED - RESONATING CAVITATING JETS AT U.C.S.D. ( A.T. ELLIS) 
TEST CELL
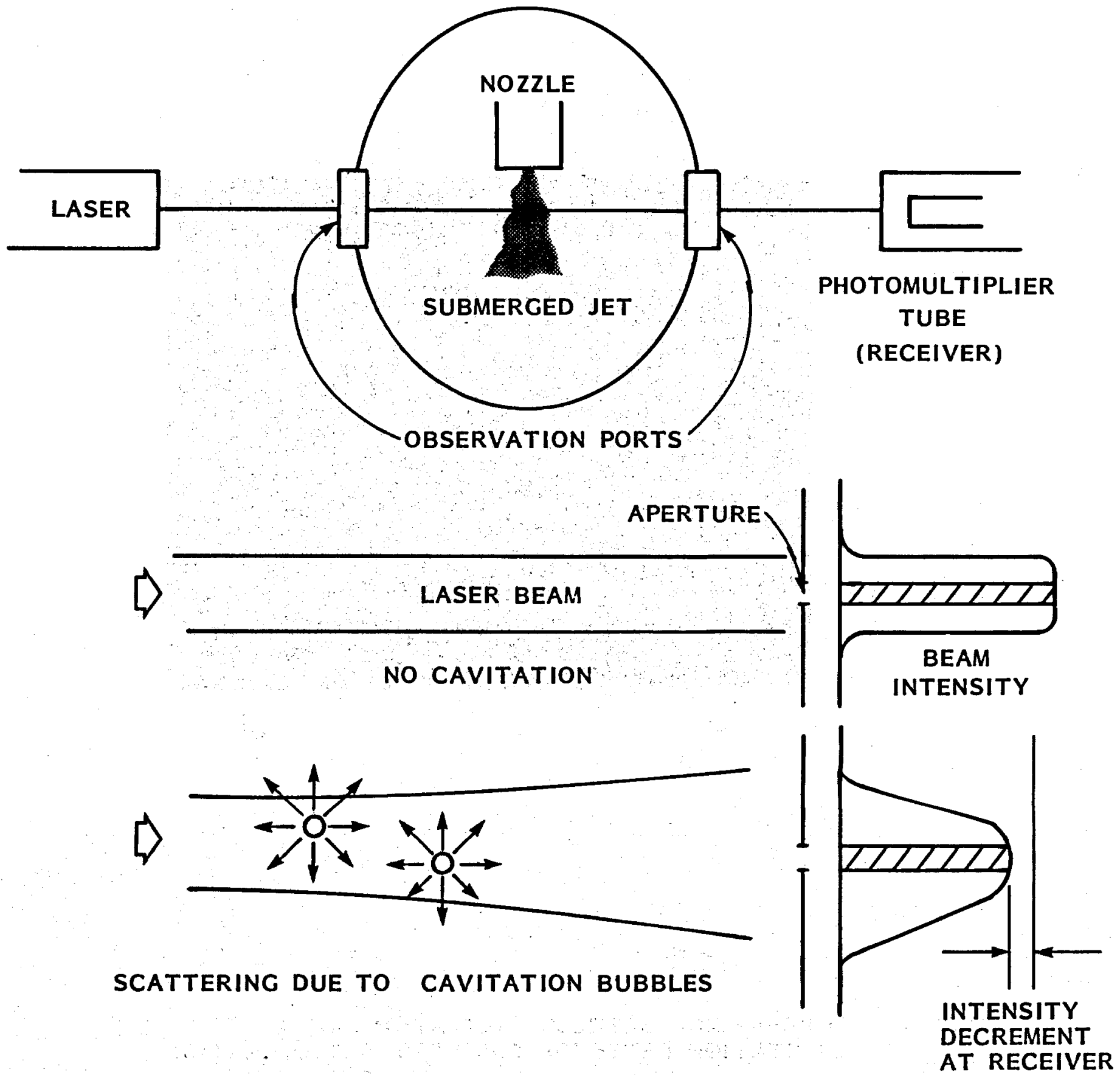

SCATTERING DUE TO CAVITATION BUBBLES

INTENSITY

AT RECEIVER

FIGURE 24 - SCHEMATIC OF LASER CAVITATION DETECTION TECHNIQUE 


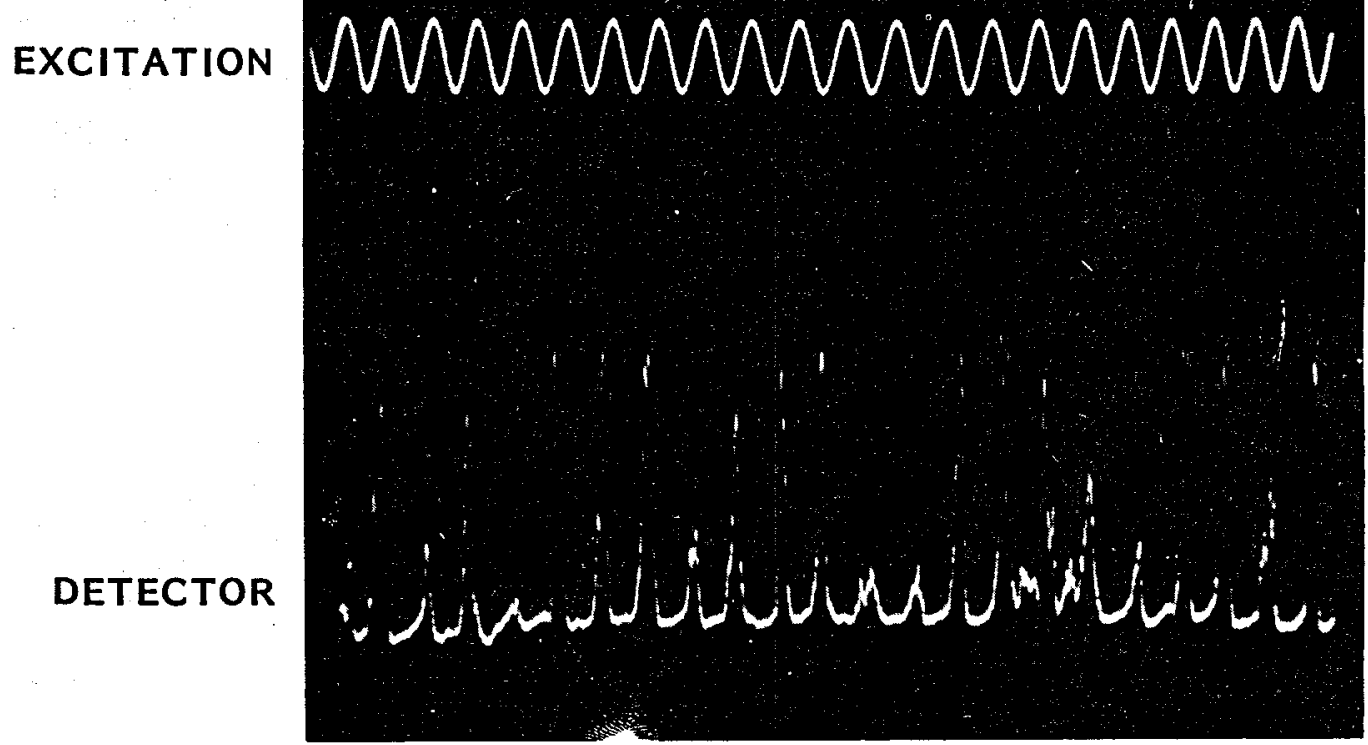

FIGURE 25 - CORRELATION BETWEEN EXCITATION AND LASER CAVITATION DETECTOR FOR RESONANT OPERATION 


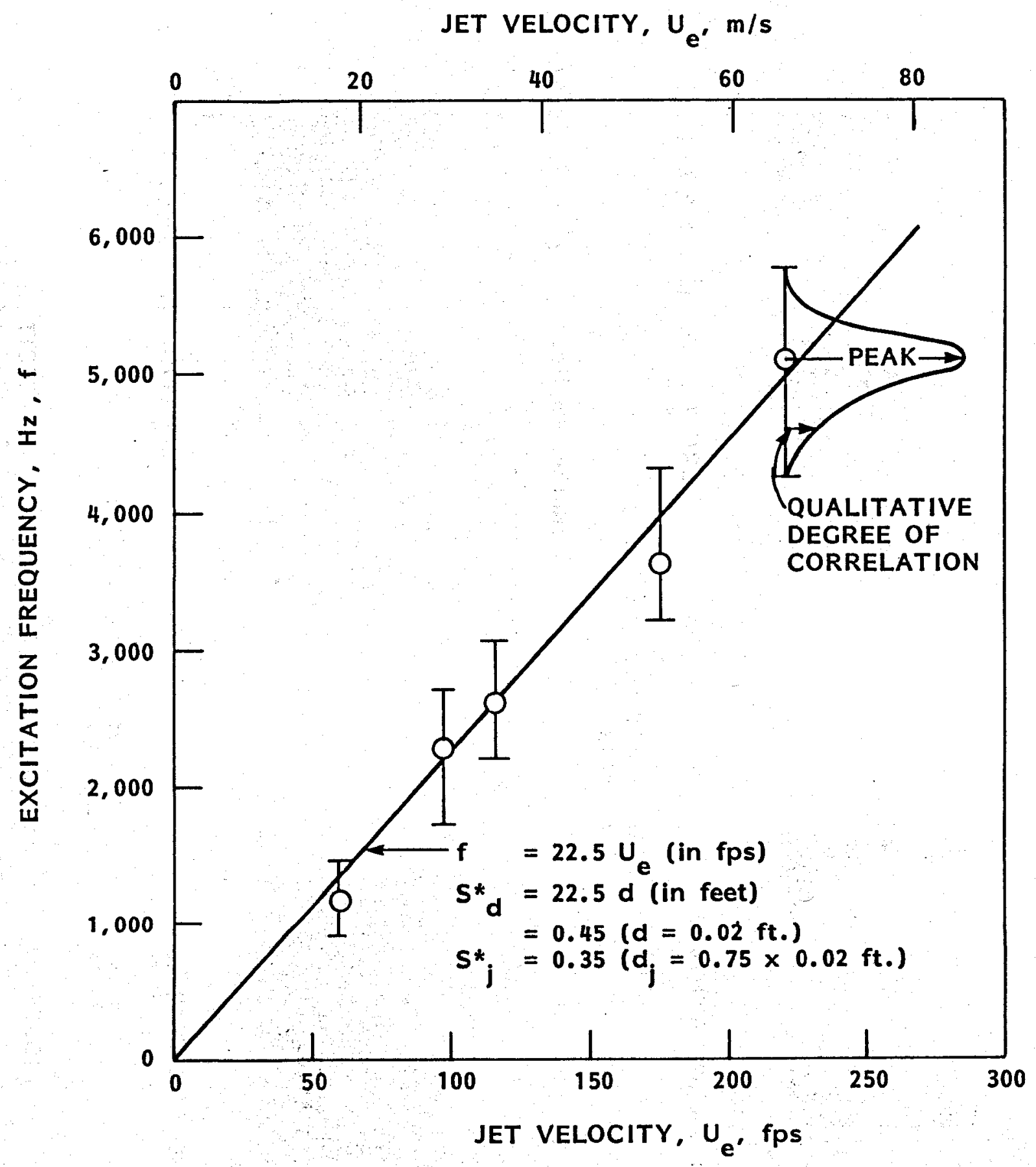

FIGURE 26 - THE INFLUENCE OF VELOCITY AND EXCITATION FREQUENCY ON THE FORMATION OF DISCRETE VORTICES 


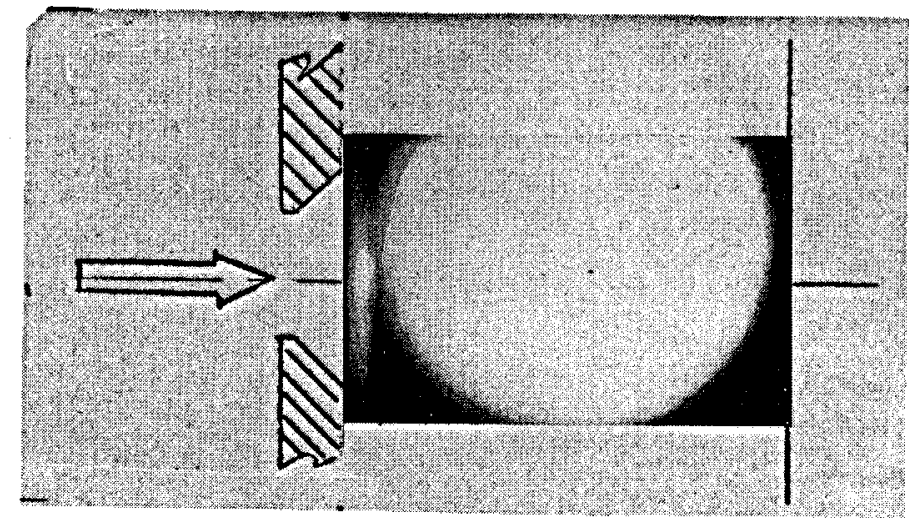

NO EXCITATION

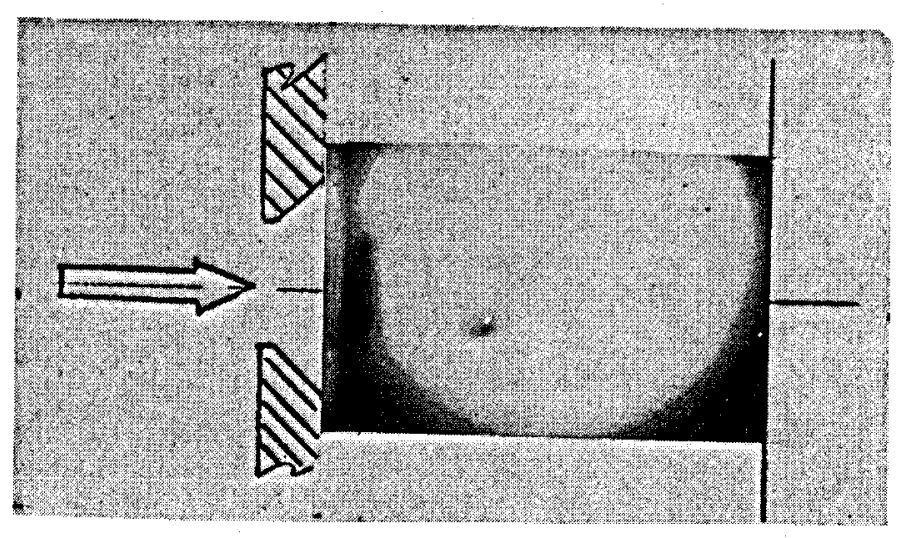

EXCITATION (f $=2,225 \mathrm{~Hz})$

a. $U=29.35 \mathrm{~m} / \mathrm{s}(95.3 \mathrm{fps})$

$\sigma=0.43$

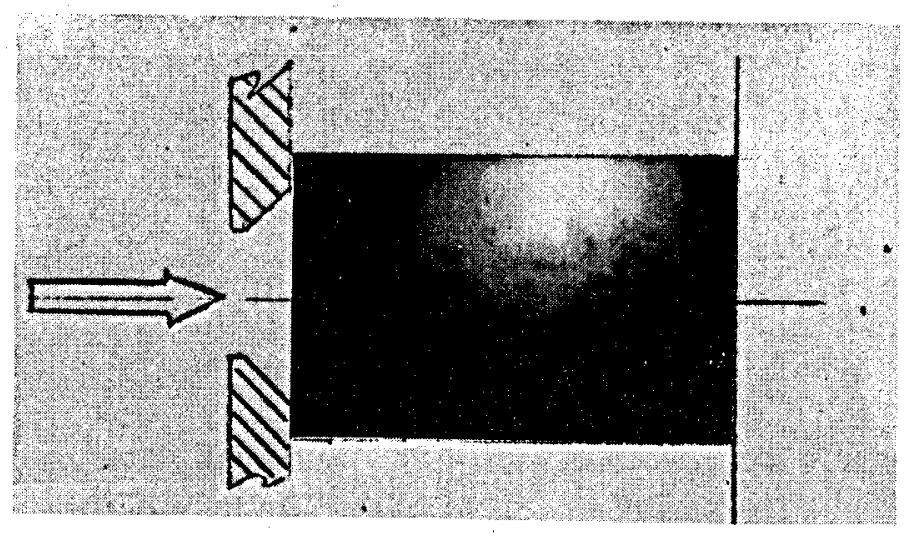

NO EXCITATION
Excitation Amplitude, $p^{\prime} / \frac{1}{2} \rho U_{0}^{2} \cong 0.02$

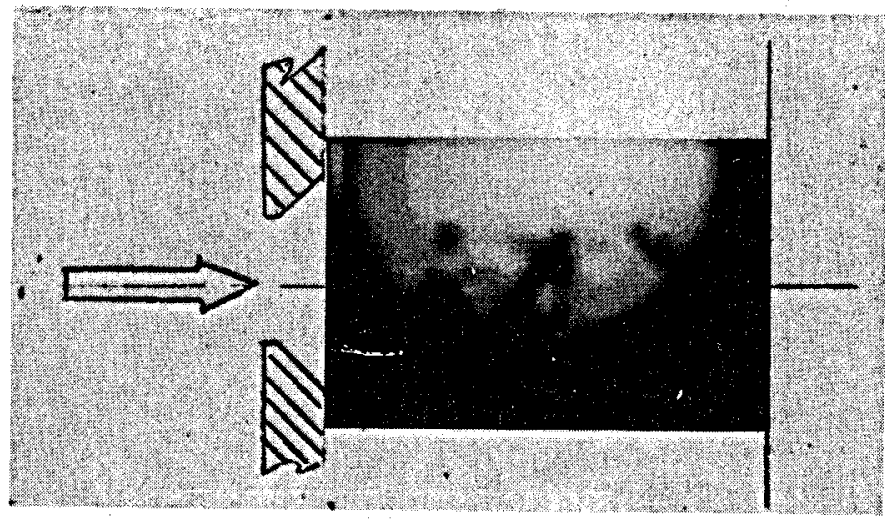

EXCITATION (f $=3,065 \mathrm{~Hz})$

b. $U=40.05 \mathrm{~m} / \mathrm{s}(131.4 \mathrm{fps})$

$\sigma=0.34$

FIGURE 27 - PHOTOGRAPHS OF EXCITED AND UNEXCITED

CAVITATING JETS (6.3 $\mathrm{mm}$ DIAMETER CAVIJET) 


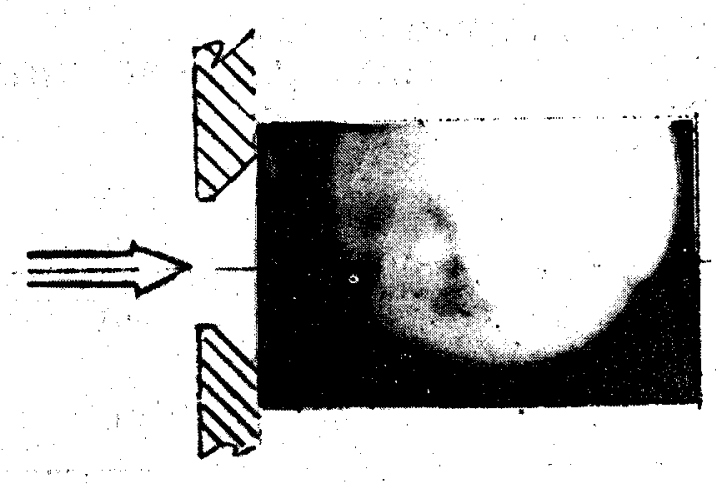

NO EXCITATION

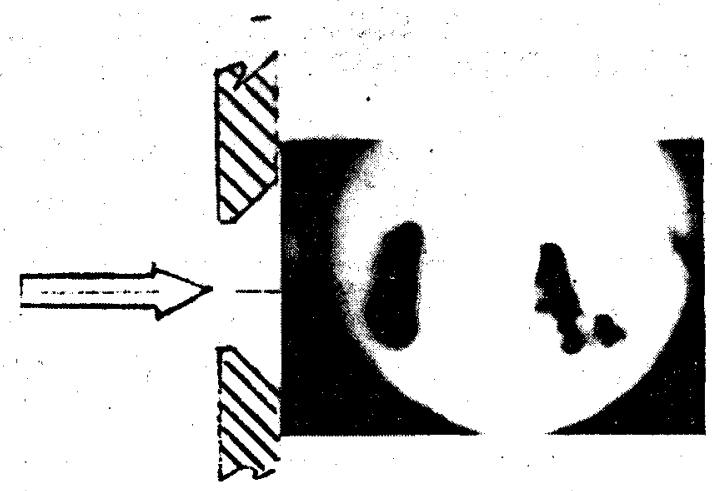

EXCITATION (f $=3,966 \mathrm{~Hz}$ )

c. $U=51.82 \mathrm{~m} / \mathrm{s}(170 \mathrm{fps})$ $\sigma=0.28$
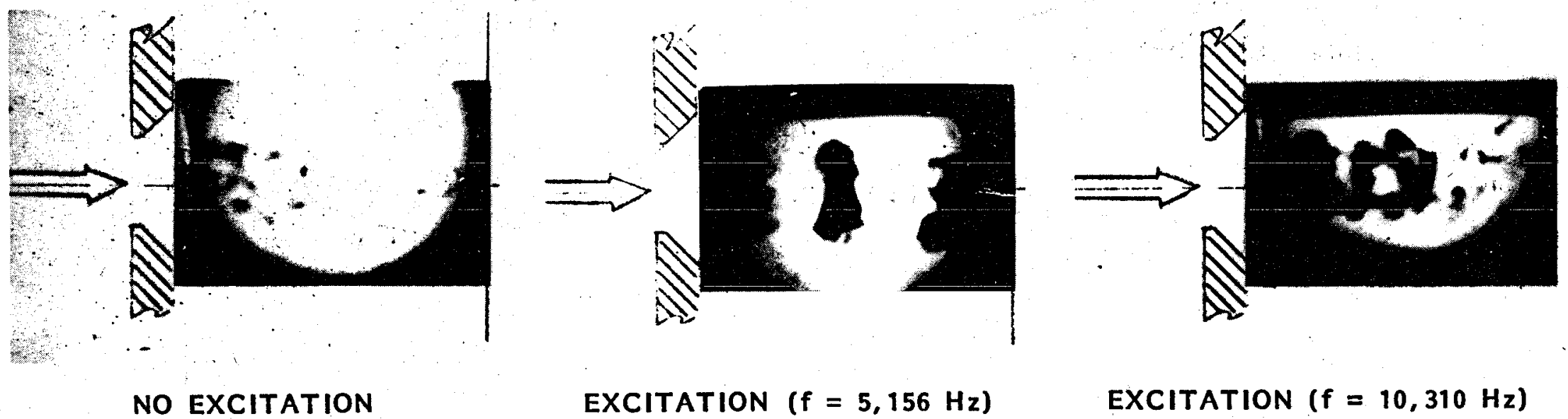

EXCITATION ( $f=5,156 \mathrm{~Hz}$ )

EXCITATION ( $f=10,310 \mathrm{~Hz}$ )

d. $U=76.36 \mathrm{~m} / \mathrm{s}(221 \mathrm{fps})$

$\sigma=0.23$

FIGURE 27 - CONCLUDED 


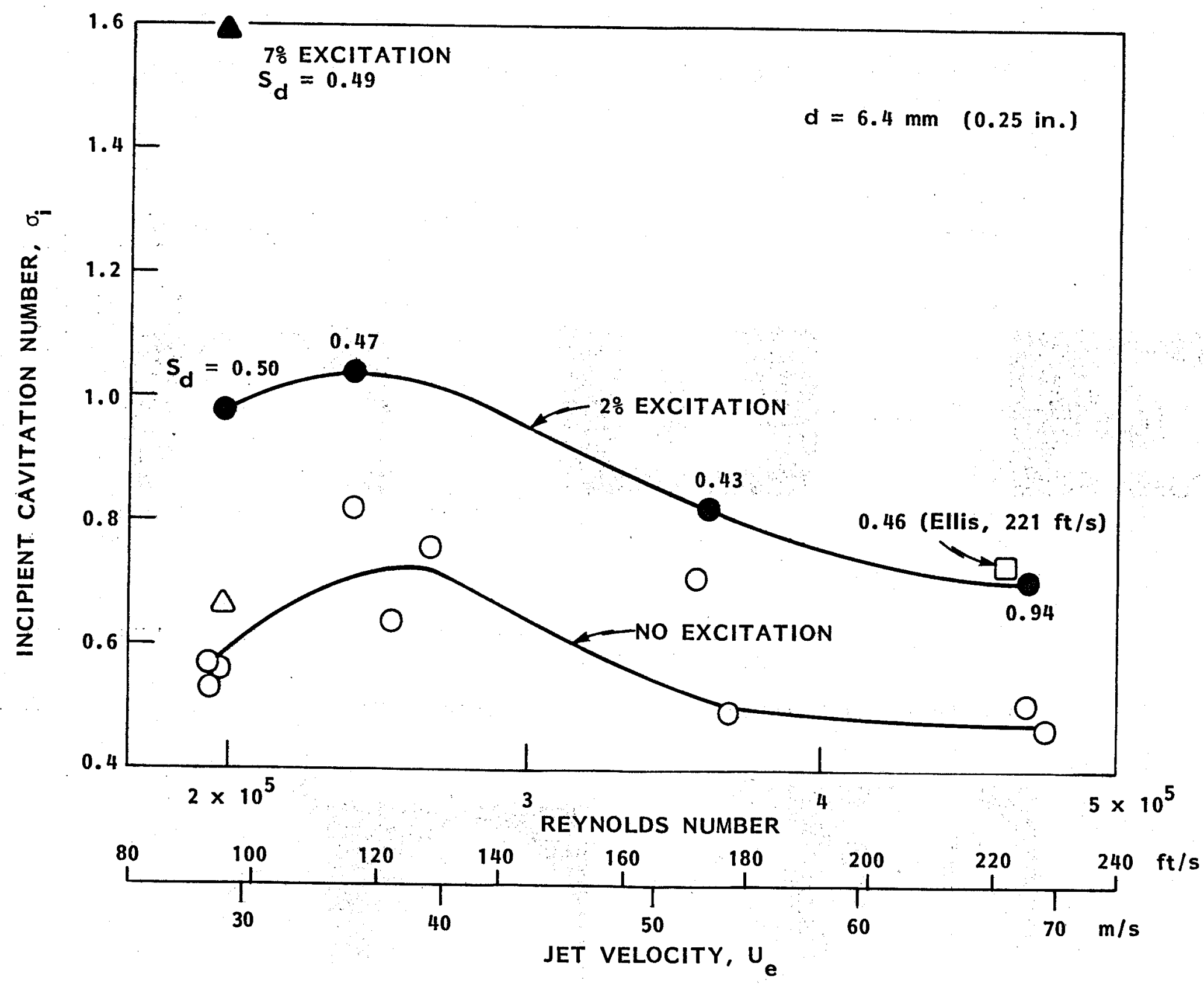

FIGURE 28 - JET CAVITATION INCEPTION FOR A PLAIN CAVIJET NOZZLE WITH AND WITHOUT EXCITATION (AT U.C.S.D.) 


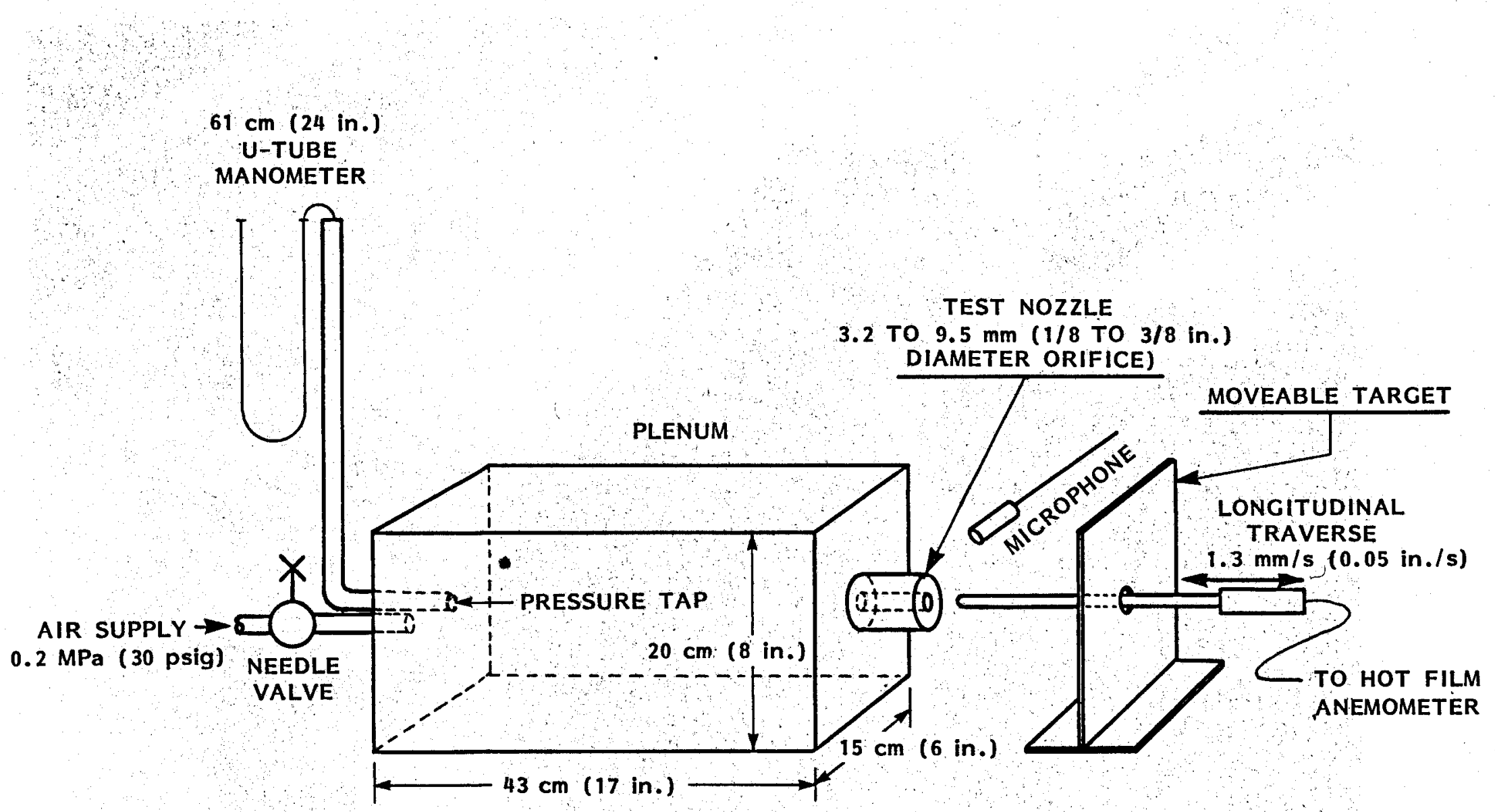

FIGURE 29 - APPARATUS FOR NOZZLE TESTS WITH AIR 


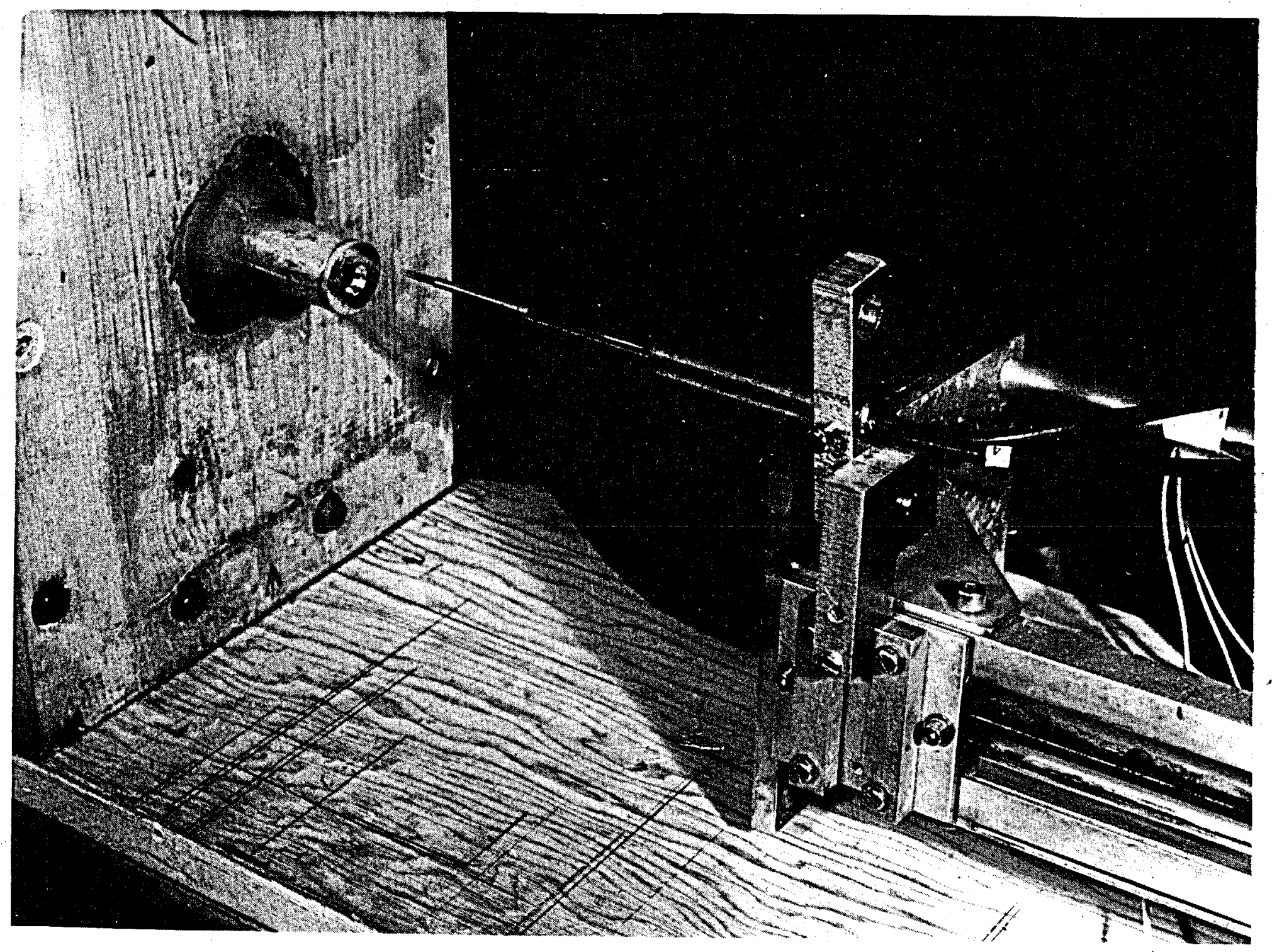

FIGURE 30 - VIEW OF VELOCITY PROBE IN THE EXITING JET FLOW 


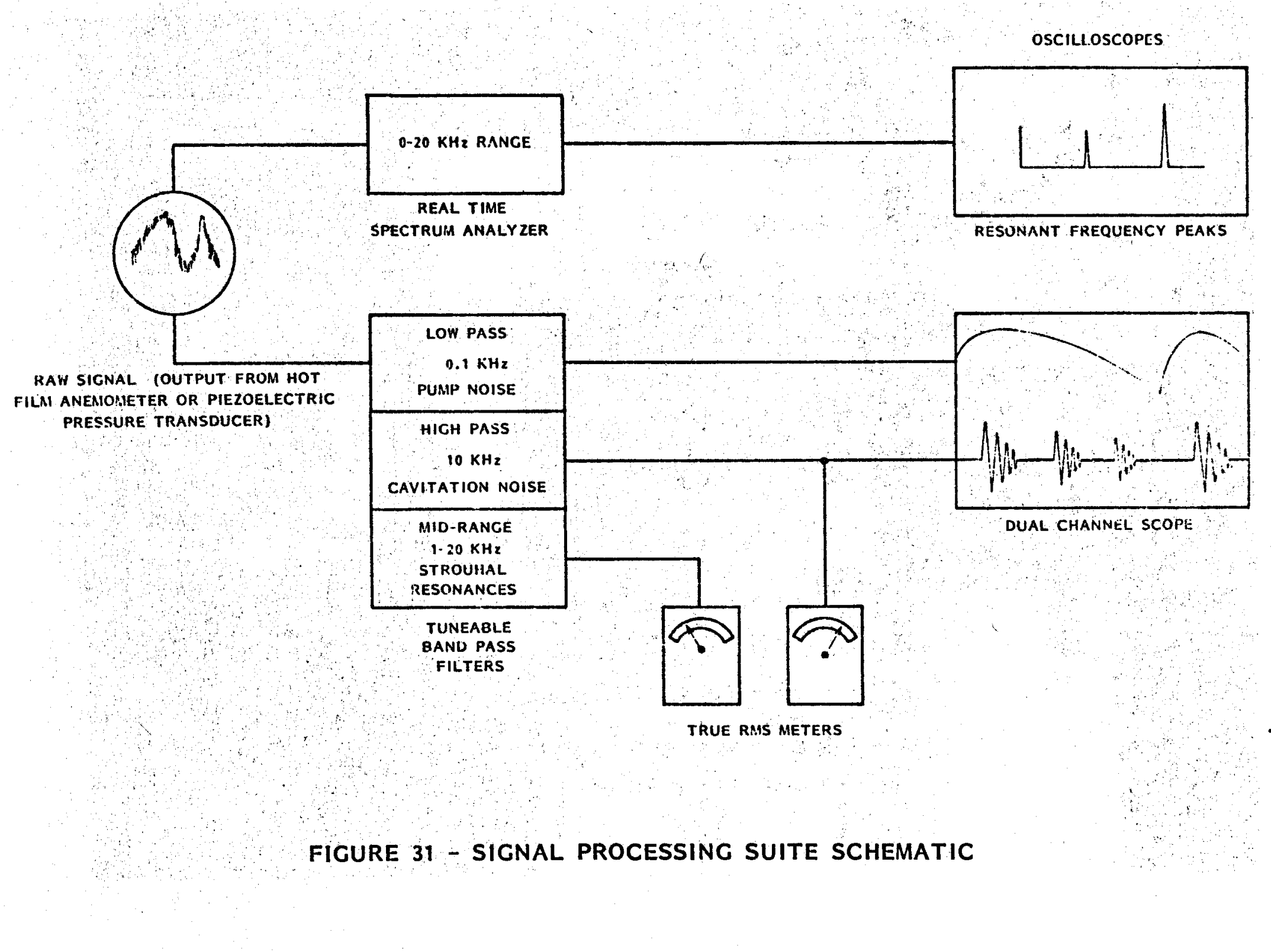




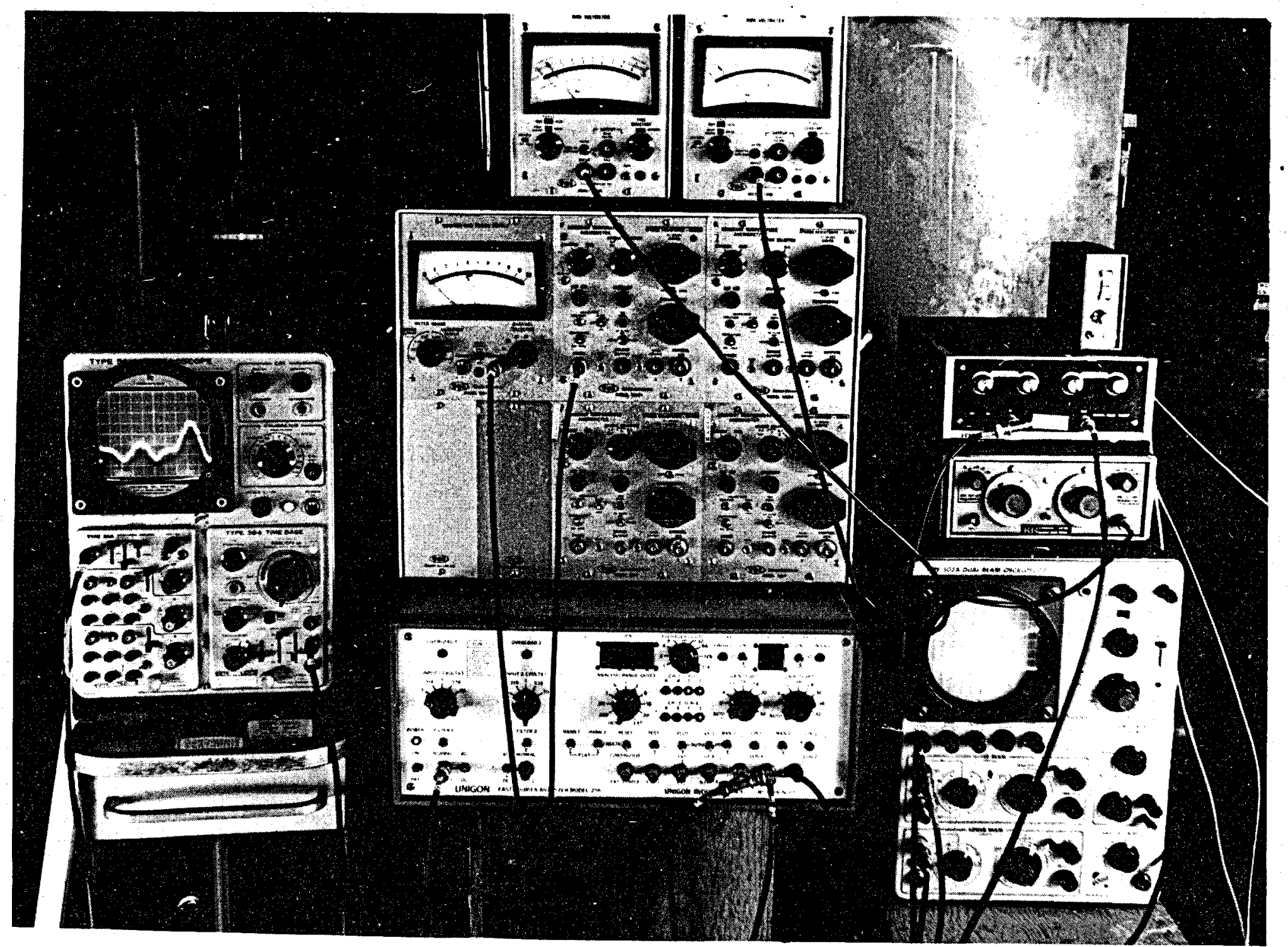

FIGURE 32 - VIEW OF INSTRUMENTATION 


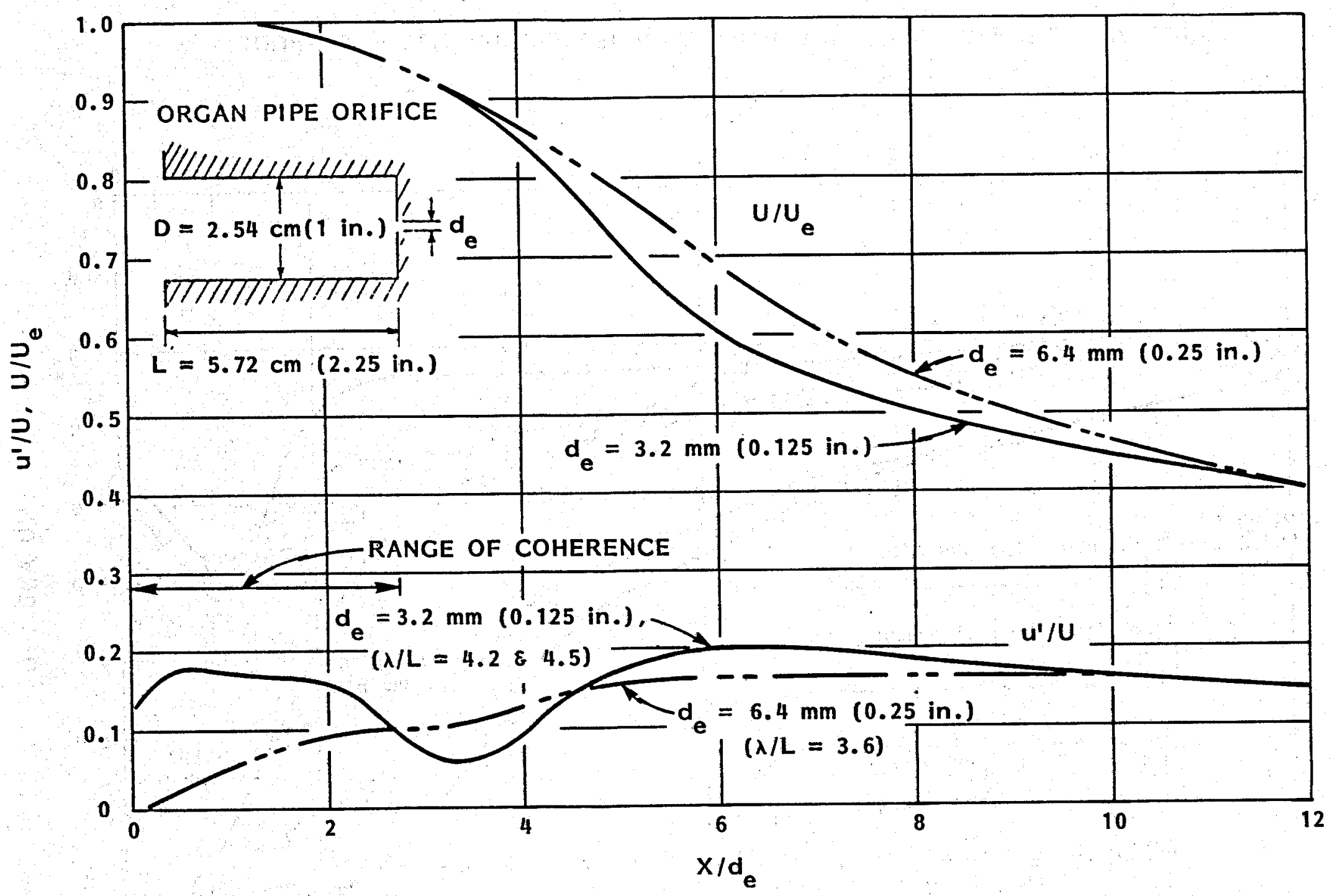

FIGURE 33 - CENTERLINE VELOCITY DISTRIBUTIONS; ORGAN PIPE RESONANT OSCILLATOR 


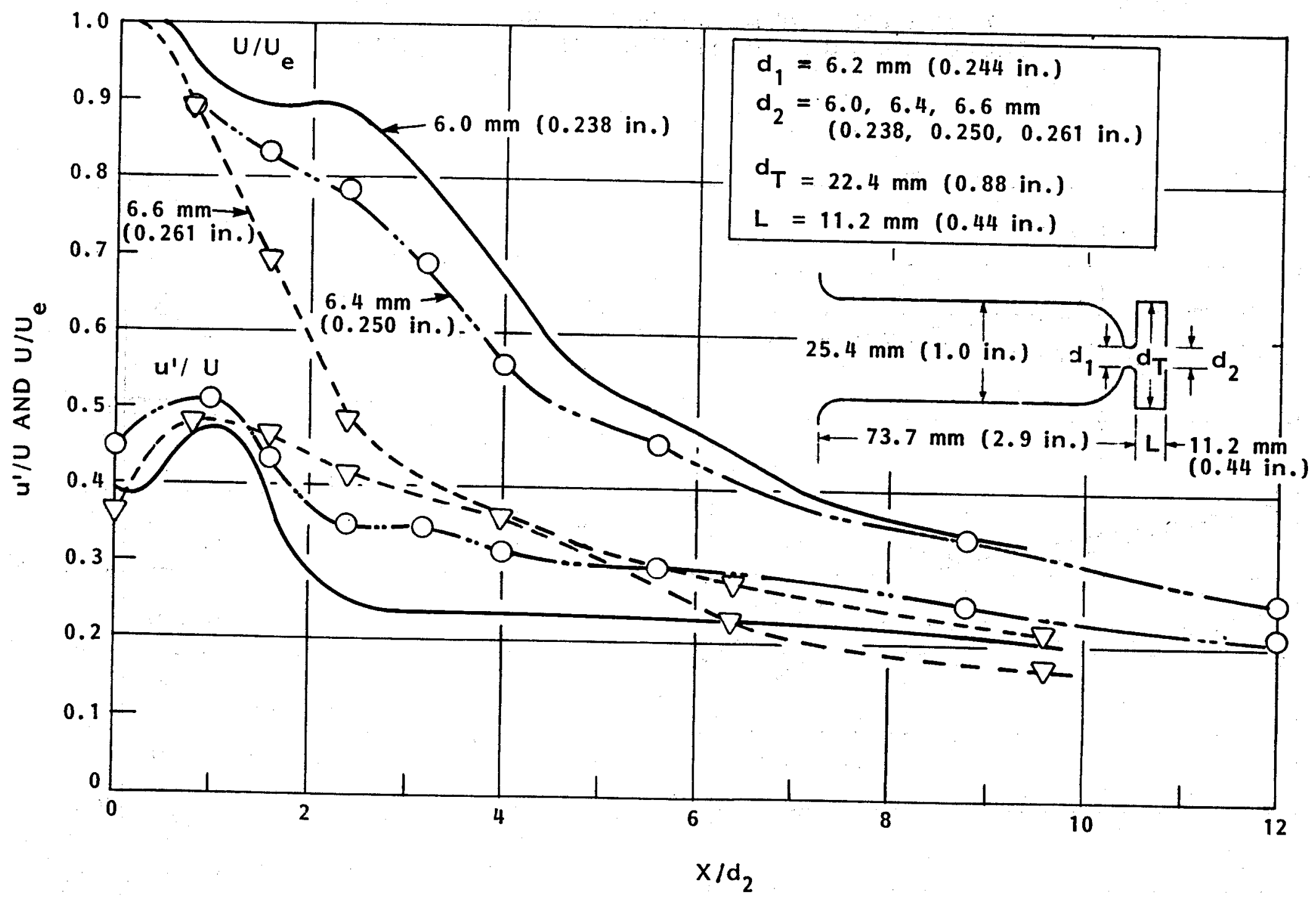

FIGURE 34 - CENTERLINE VELOCITY DISTRIBUTIONS; TYPICAL "PULSER" 


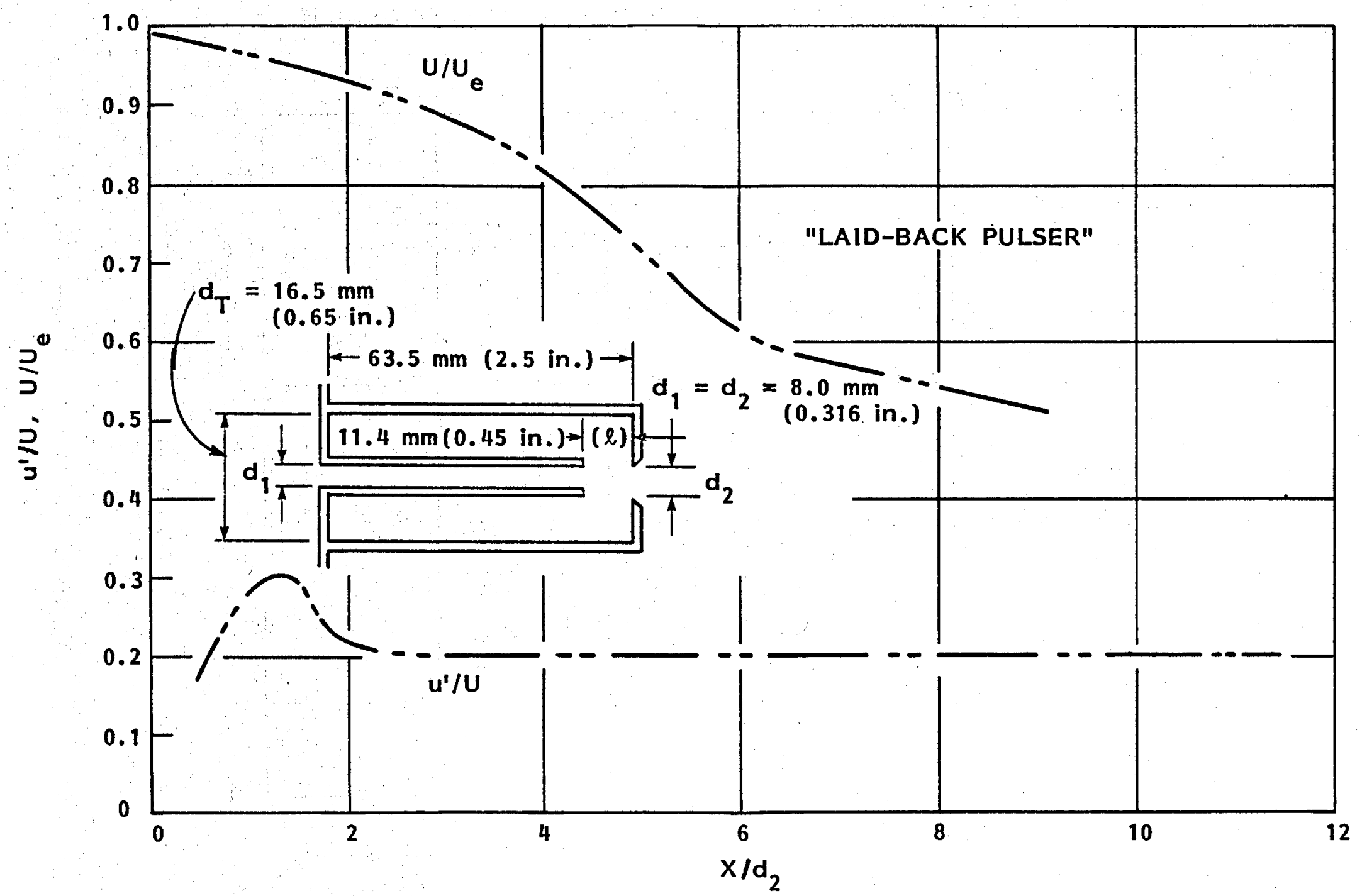

FIGURE 35 - CENTERLINE VELOCITY DISTRIBUTIONS; "LAID-BACK PULSER" 


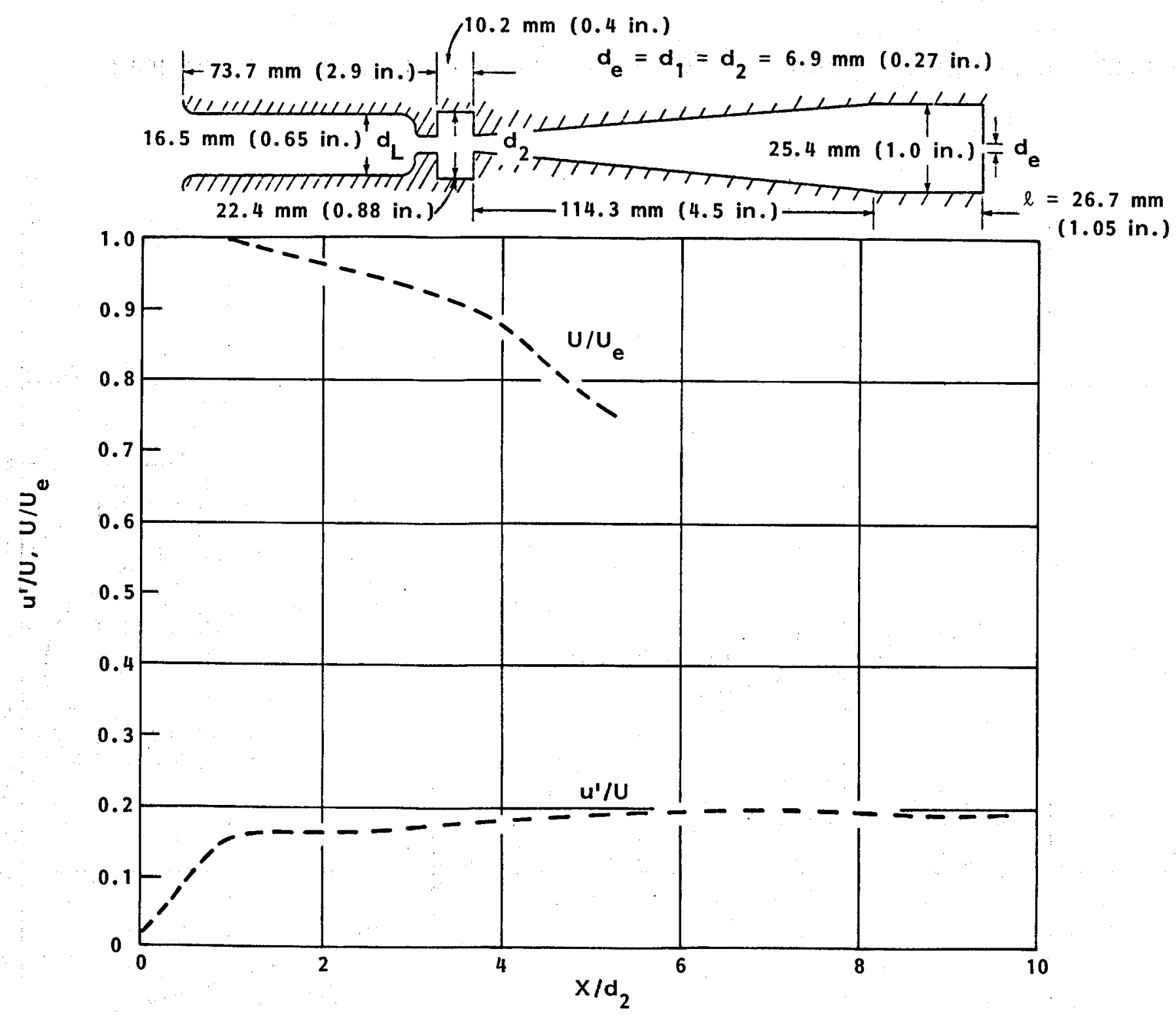

FIGURE 36 - CENTERLINE VELOCITY DISTRIBUTION; "PULSER-FED" CAVIJET (DIFFUSER) 

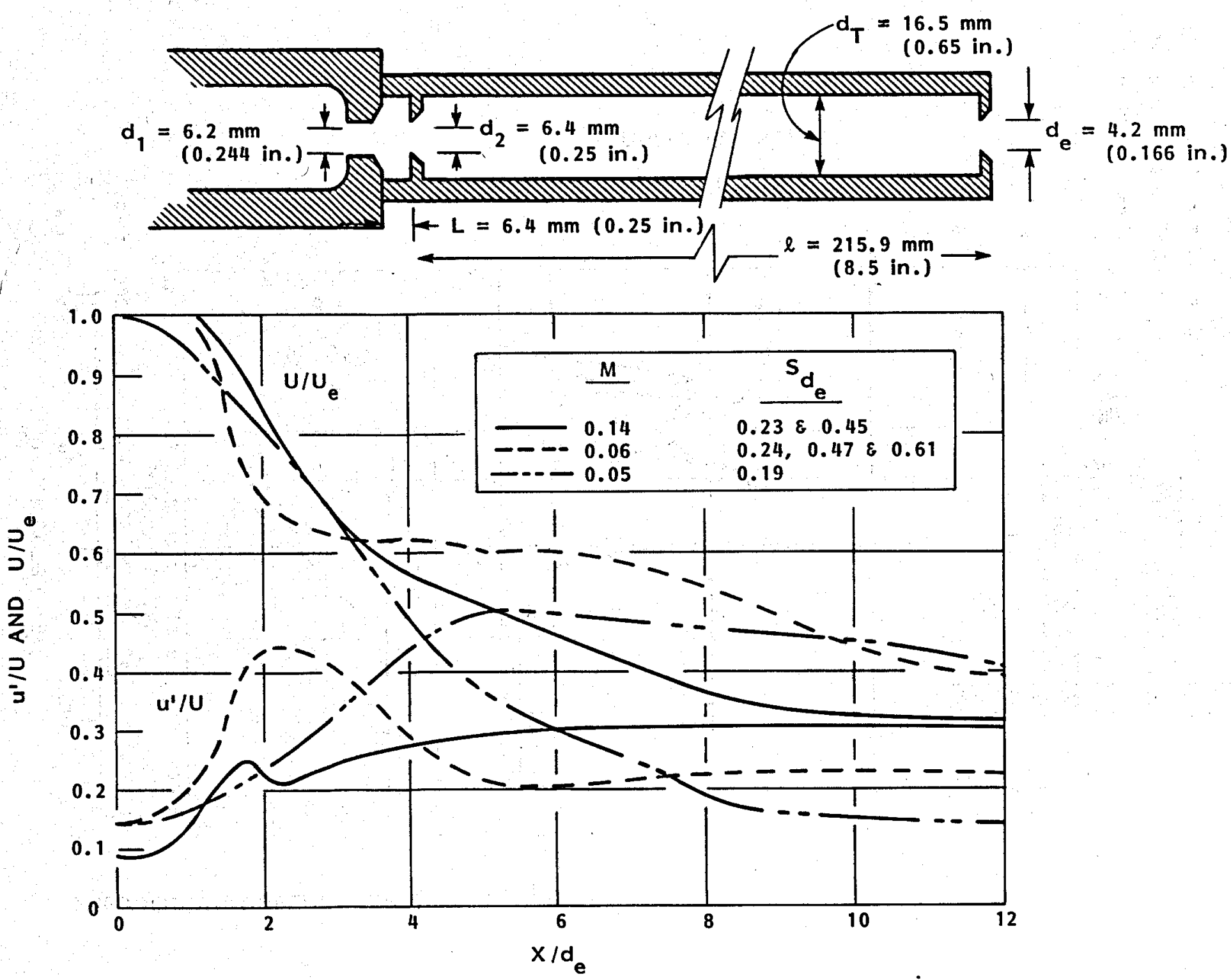

FIGURE $37^{\circ}$ - CENTER VELOCITY DISTRIBUTIONS, "PULSER-FED" CAVIJET (SUDDEN EXPANSION) 


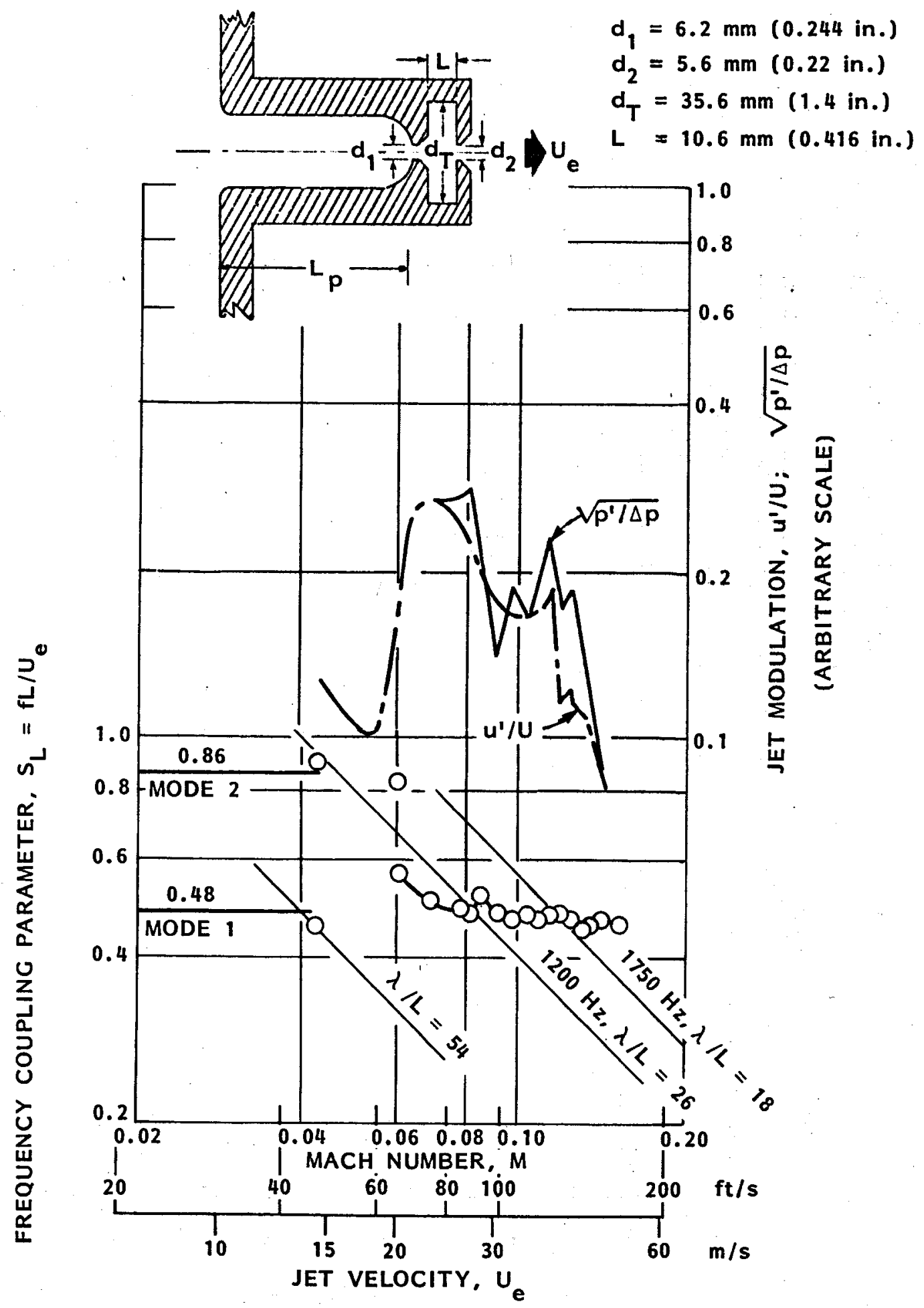

FIGURE 38 - FREQUENCY COUPLING AND MODULATION AMPLITUDES; LF PULSER IN AIR 

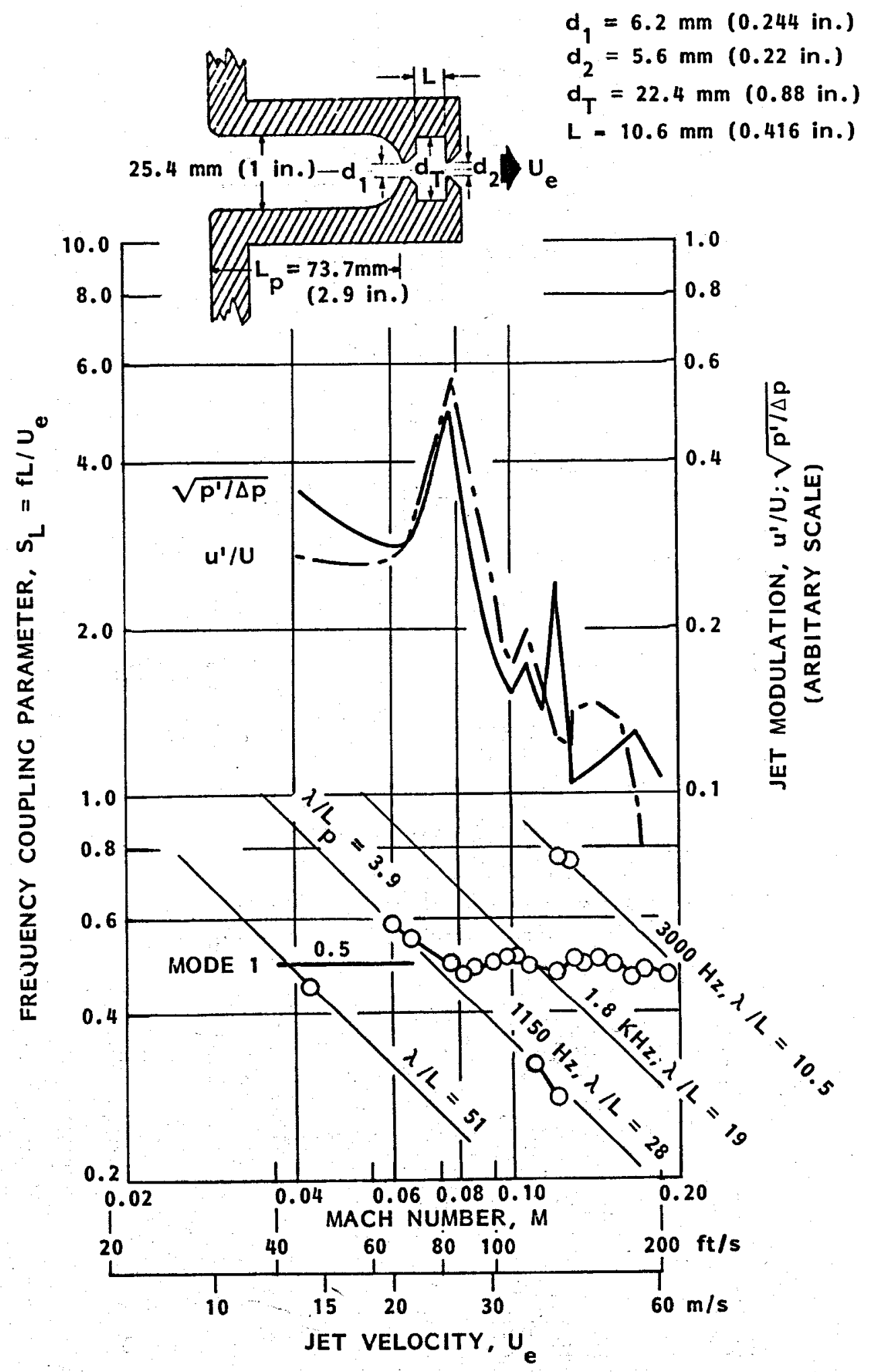

FIGURE 39 - FREQUENCY COUPLING AND MODULATION AMPLITUDES; HF PULSER IN AIR 

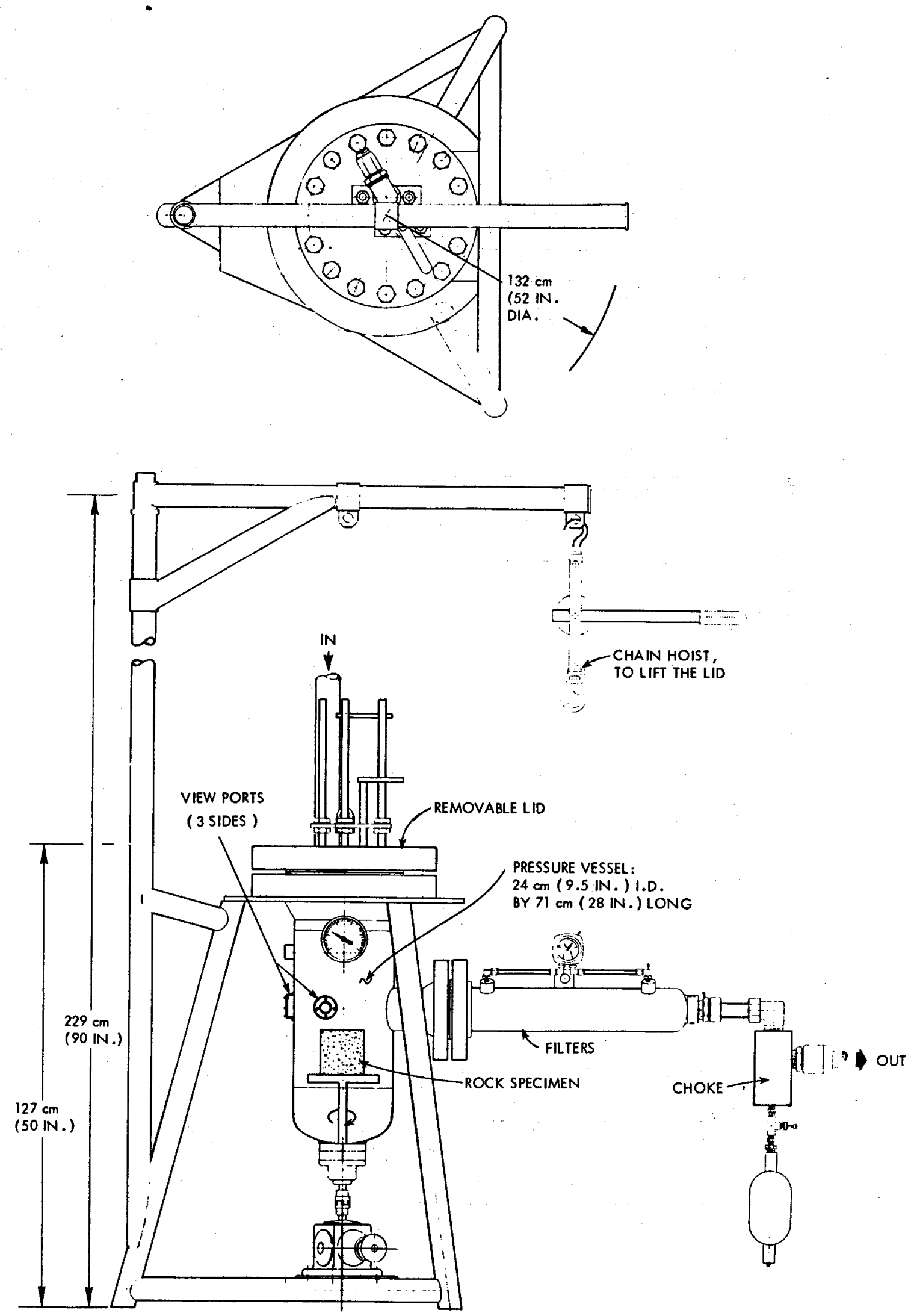

FIGURE 40 - HIGH PRESSURE CELL, HPC 


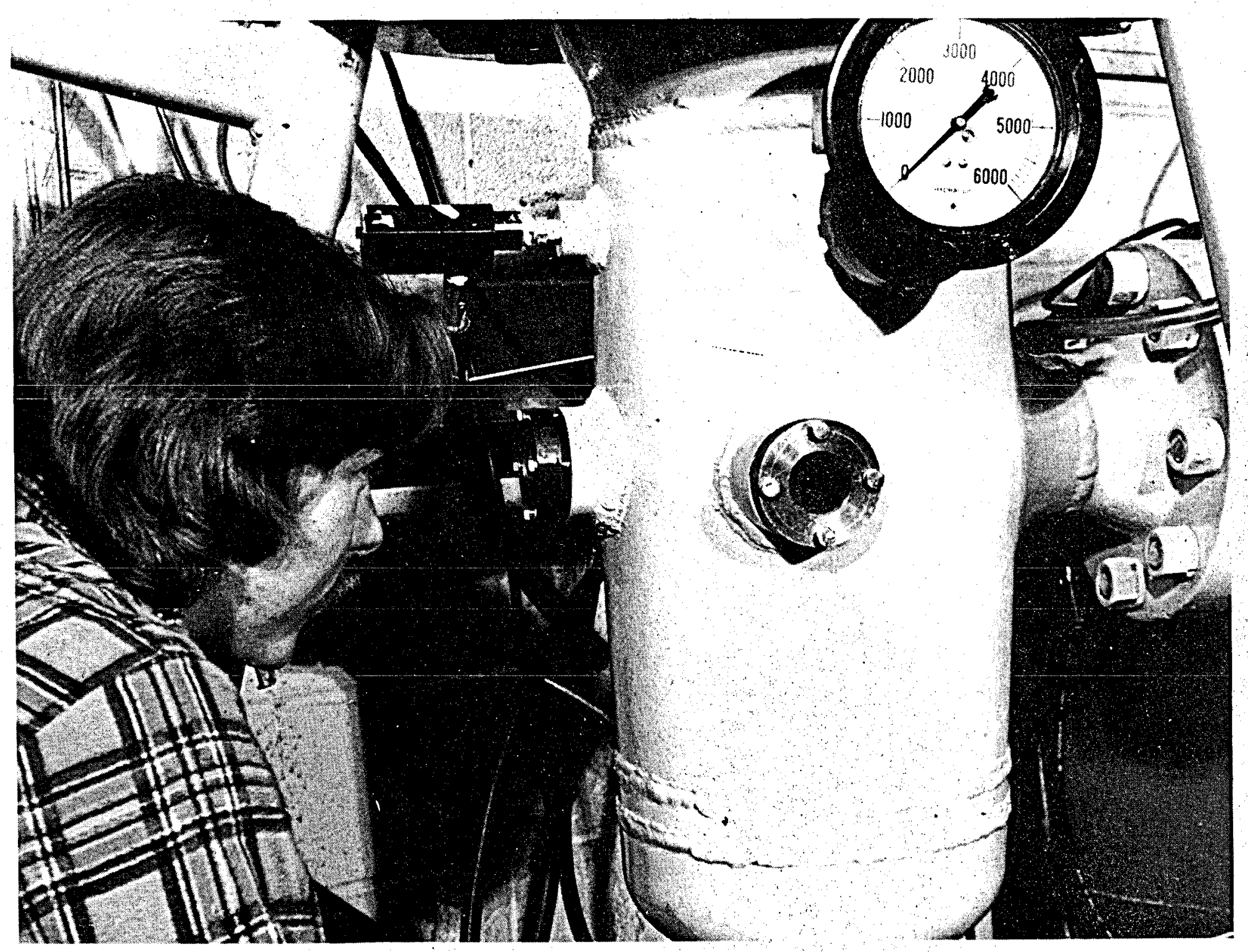

FIGURE 41 - VIEW OF HIGH PRESSURE CELL SHOWING VIEWING PORTS 


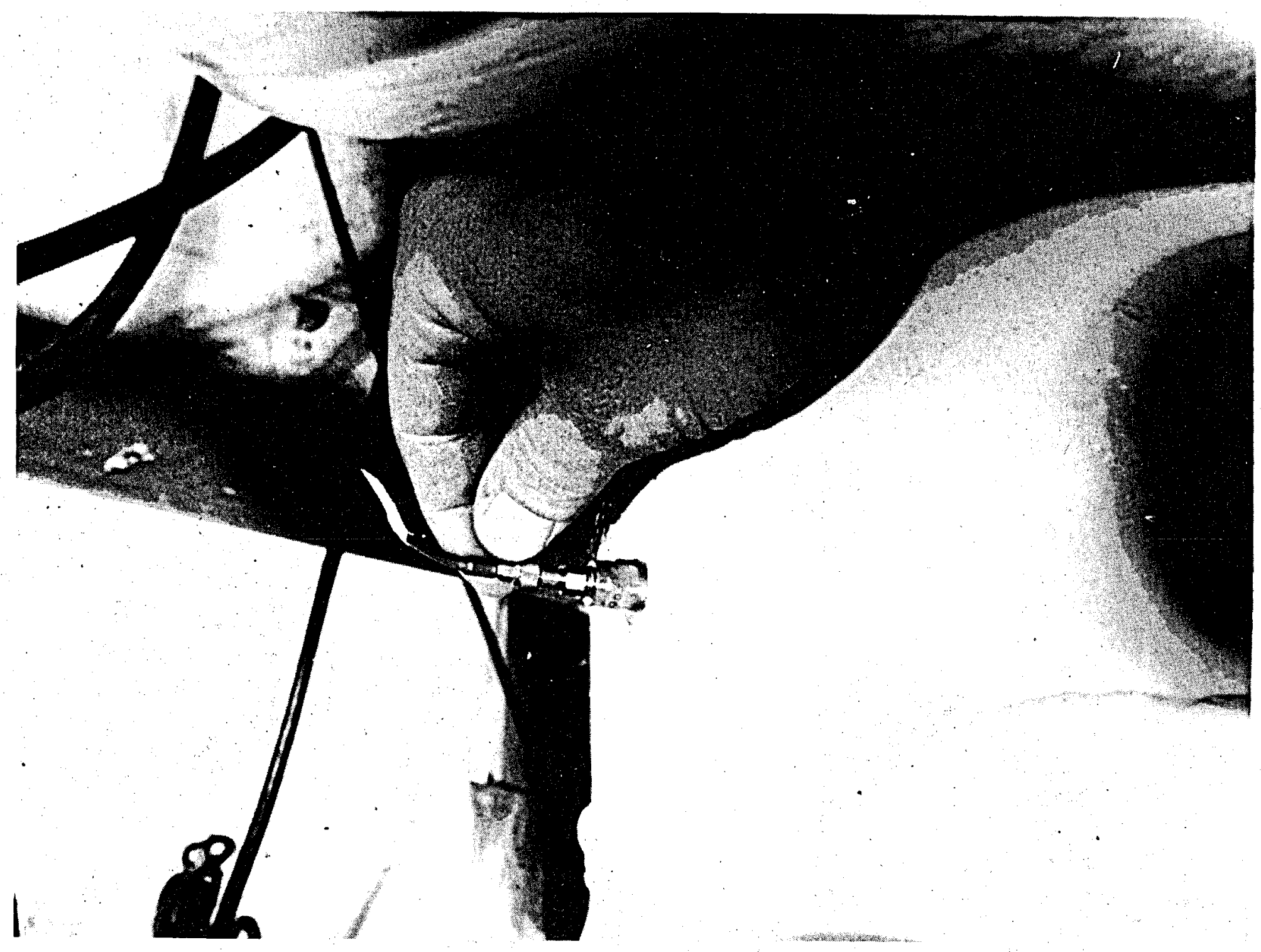

FIGURE 42 - VIEW OF PIEZOELECTRIC TRANSDUCER IN WALL OF HPC 


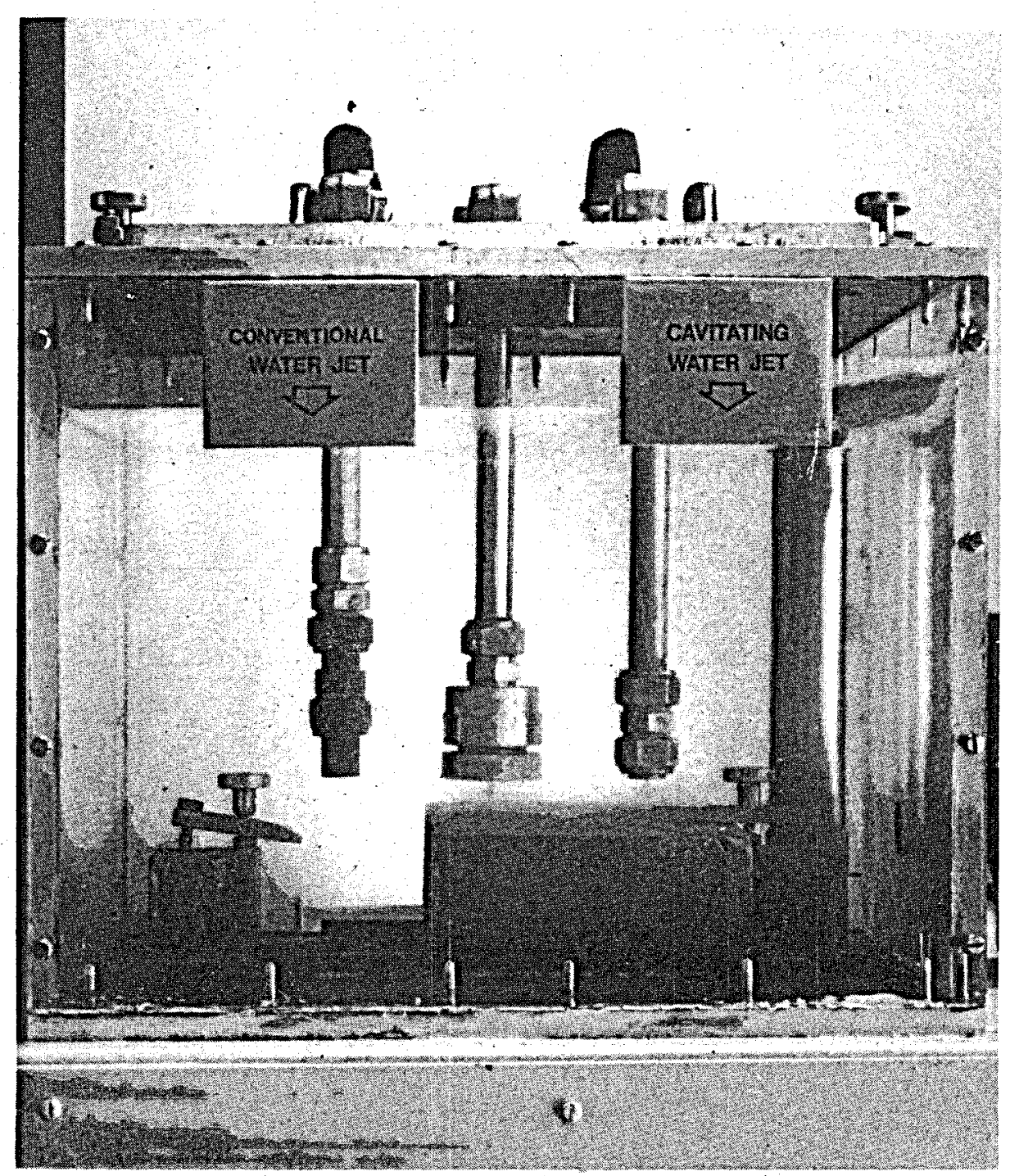

FIGURE 43 - VIEW OF CAVIJET ATMOSPHERIC PRESSURE TEST FACILITY 


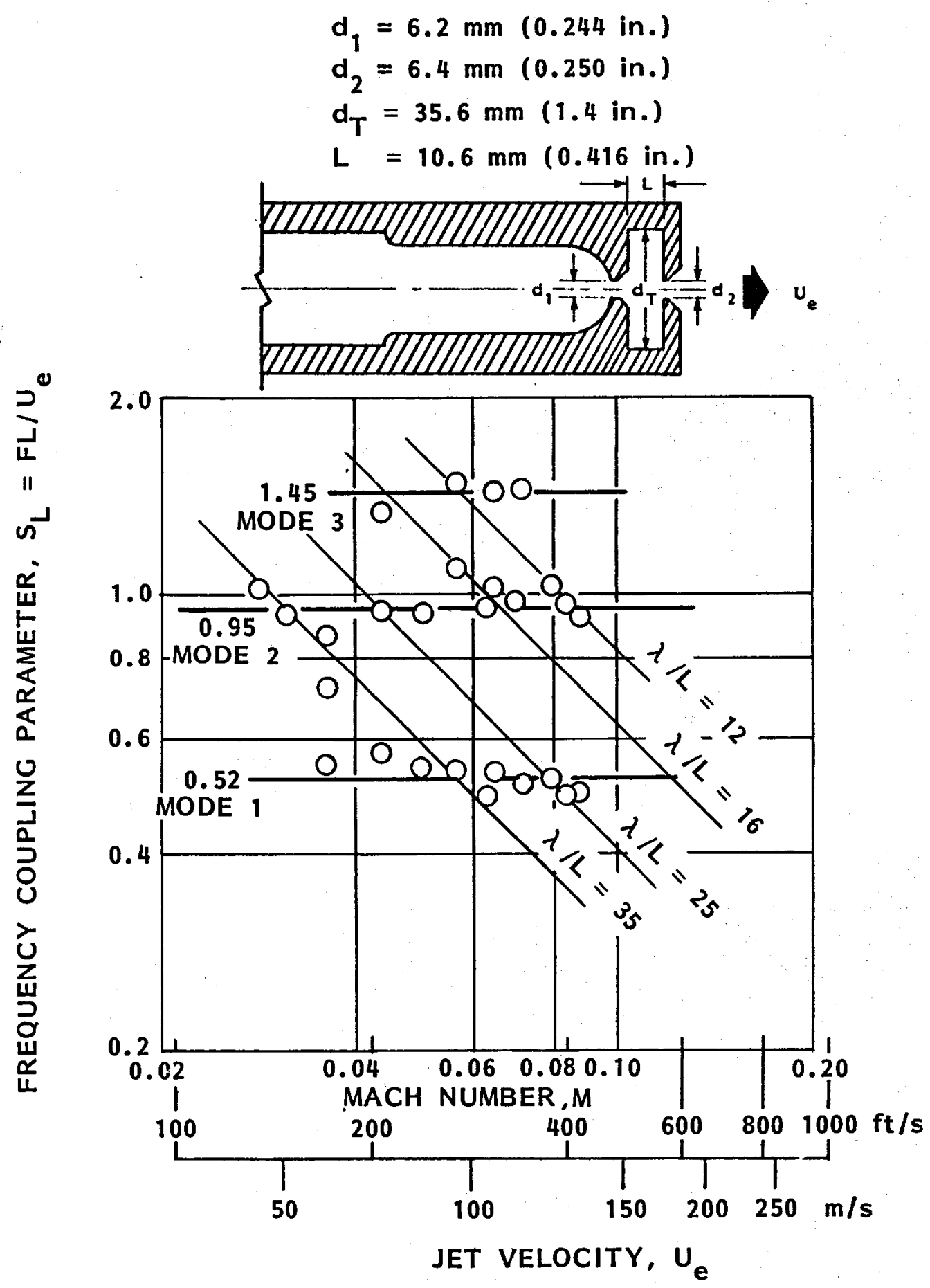

FIGURE 44 - FREQUENCY COUPLING; LF PULSER IN WATER 
$d_{1}=6.2 \mathrm{~mm}(0.244$ in.)

$d_{2}=5.6 \mathrm{~mm}(0.220 \mathrm{in.})$

$\mathrm{d}_{\mathrm{T}}=22.4 \mathrm{~mm}$ (0.88 in.)

$L=10.6 \mathrm{~mm}$ (0.416 in.)

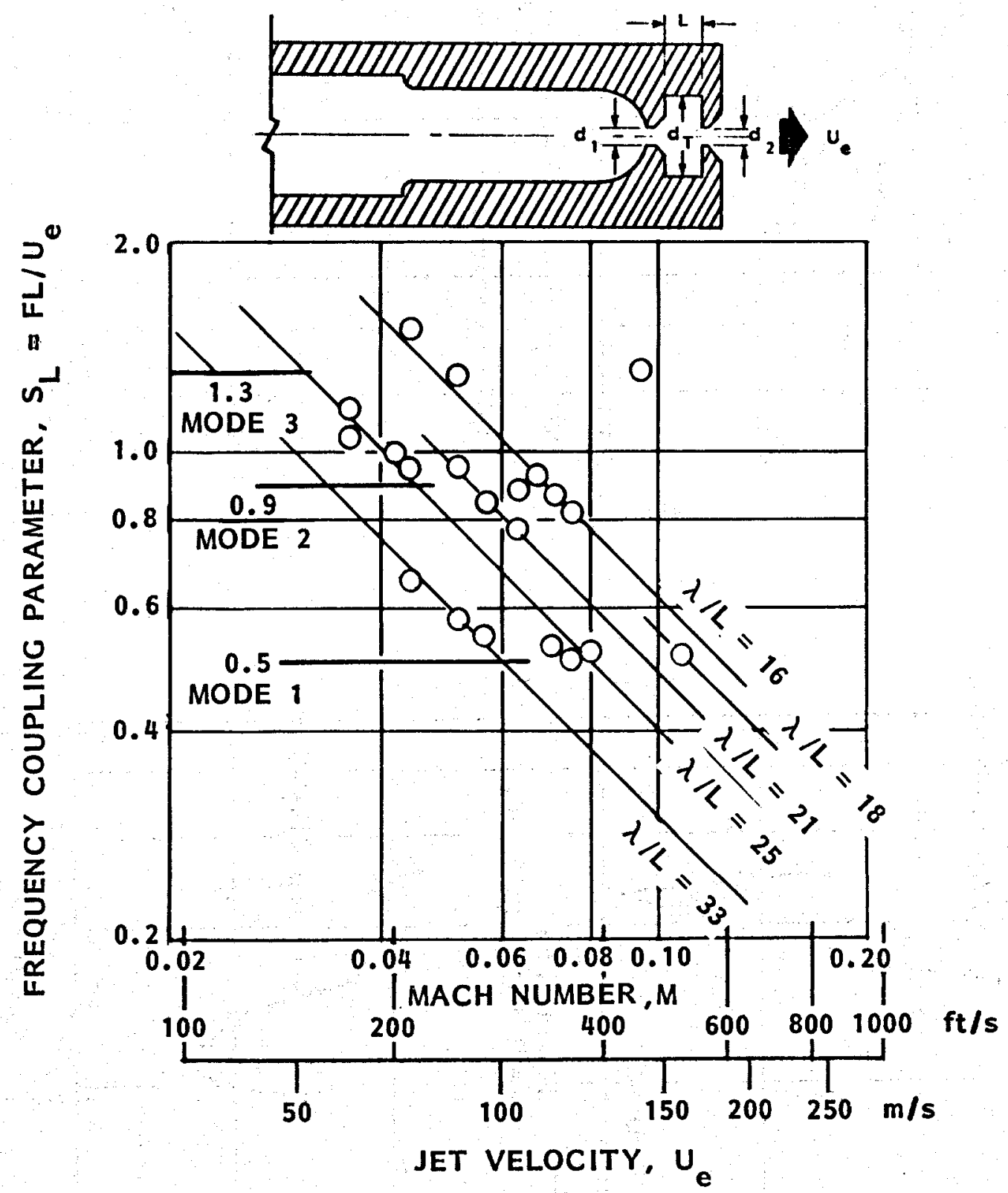

FIGURE 45 - FREQUENCY COUPLING; HF PULSER IN WATER 

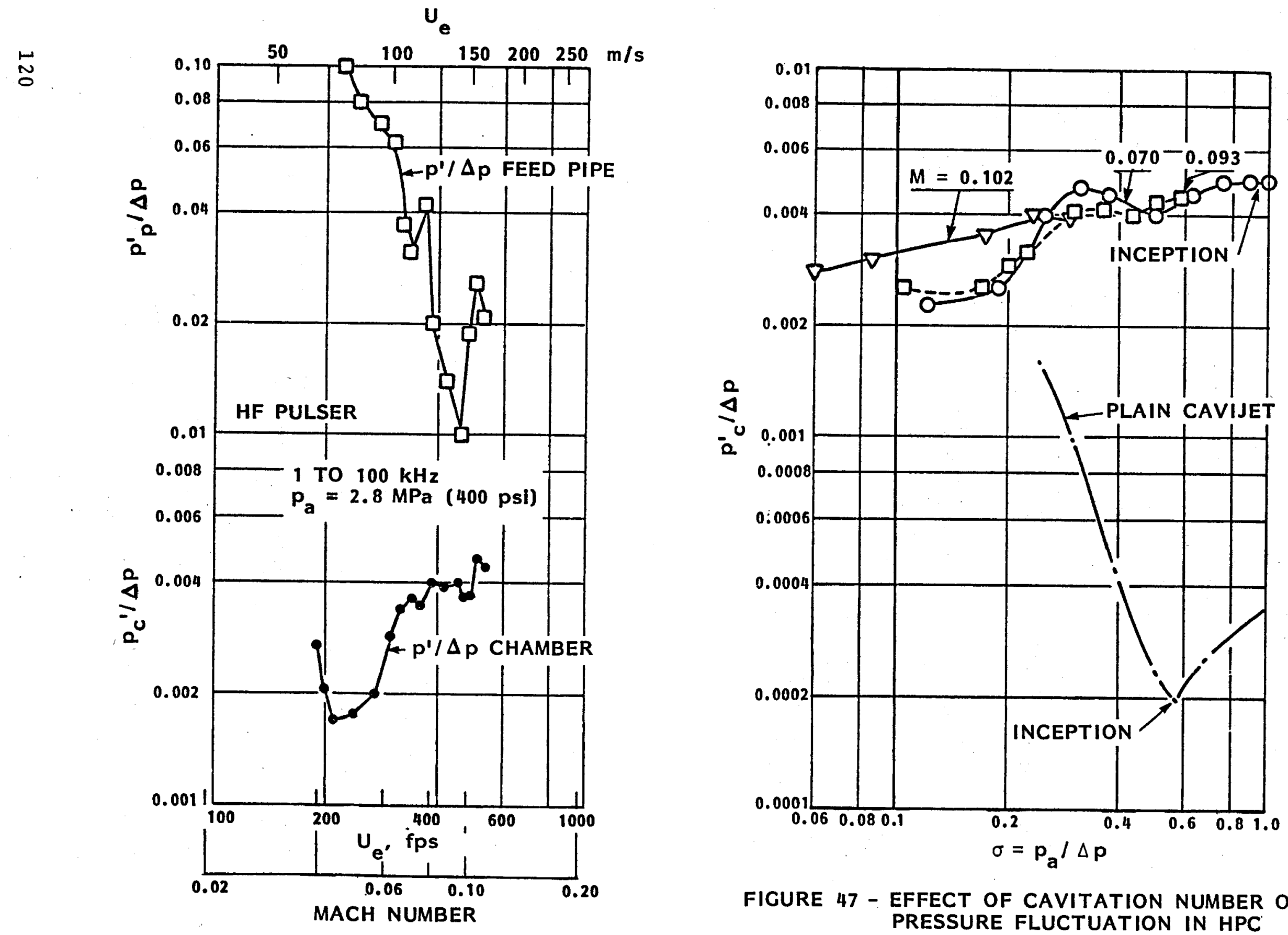

\section{FIGURE 47 - EFFECT OF CAVITATION NUMBER ON} PRESSURE FLUCTUATION IN HPC

FIGURE 46 - PRESSURE FLUCTUATIONS IN HPC, HF PULSER 


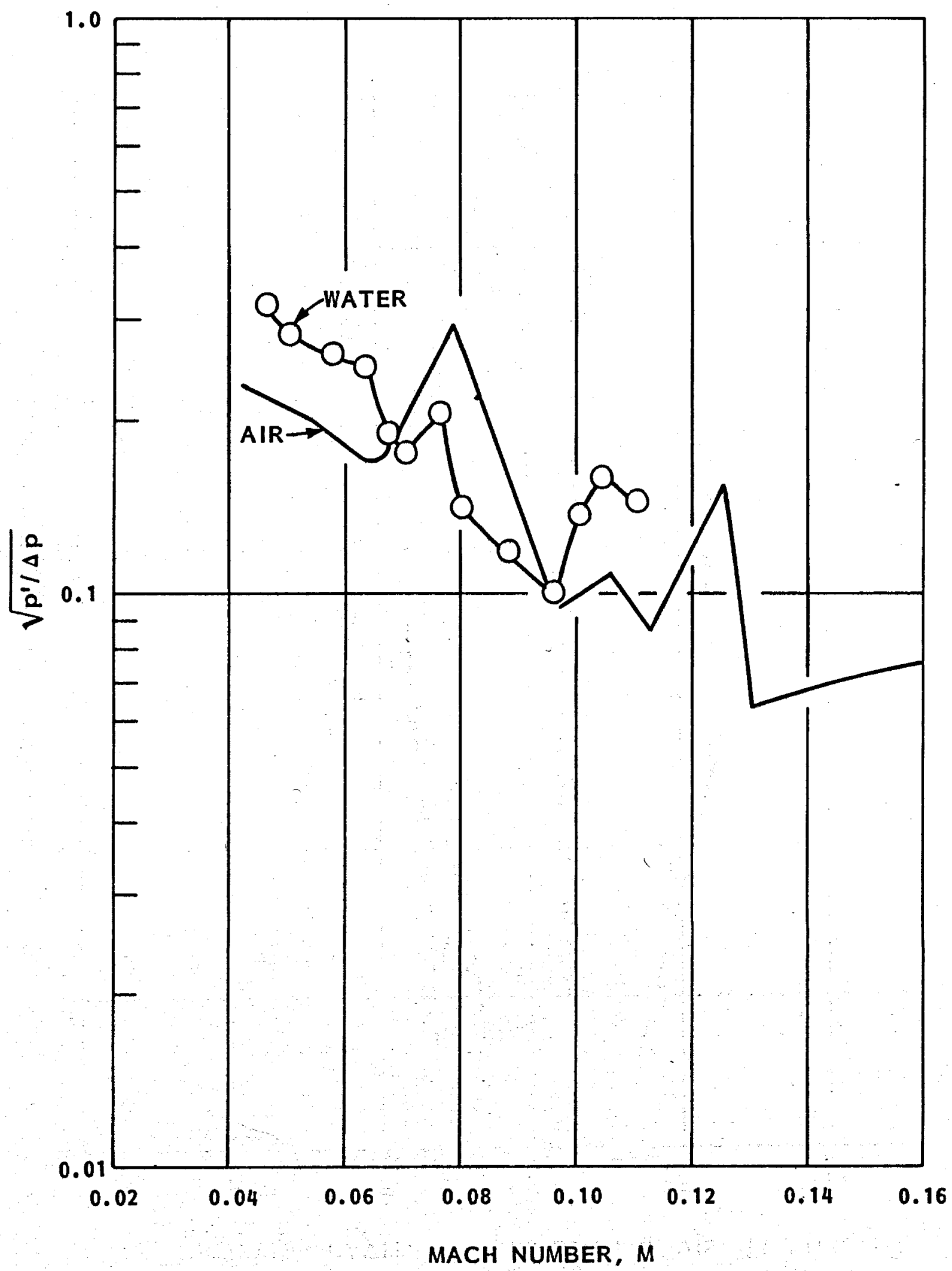

FIGURE 48 - COMPARISON OF HF PULSER PRESSURE MODULATIONS MEASURED IN AIR AND WATER 


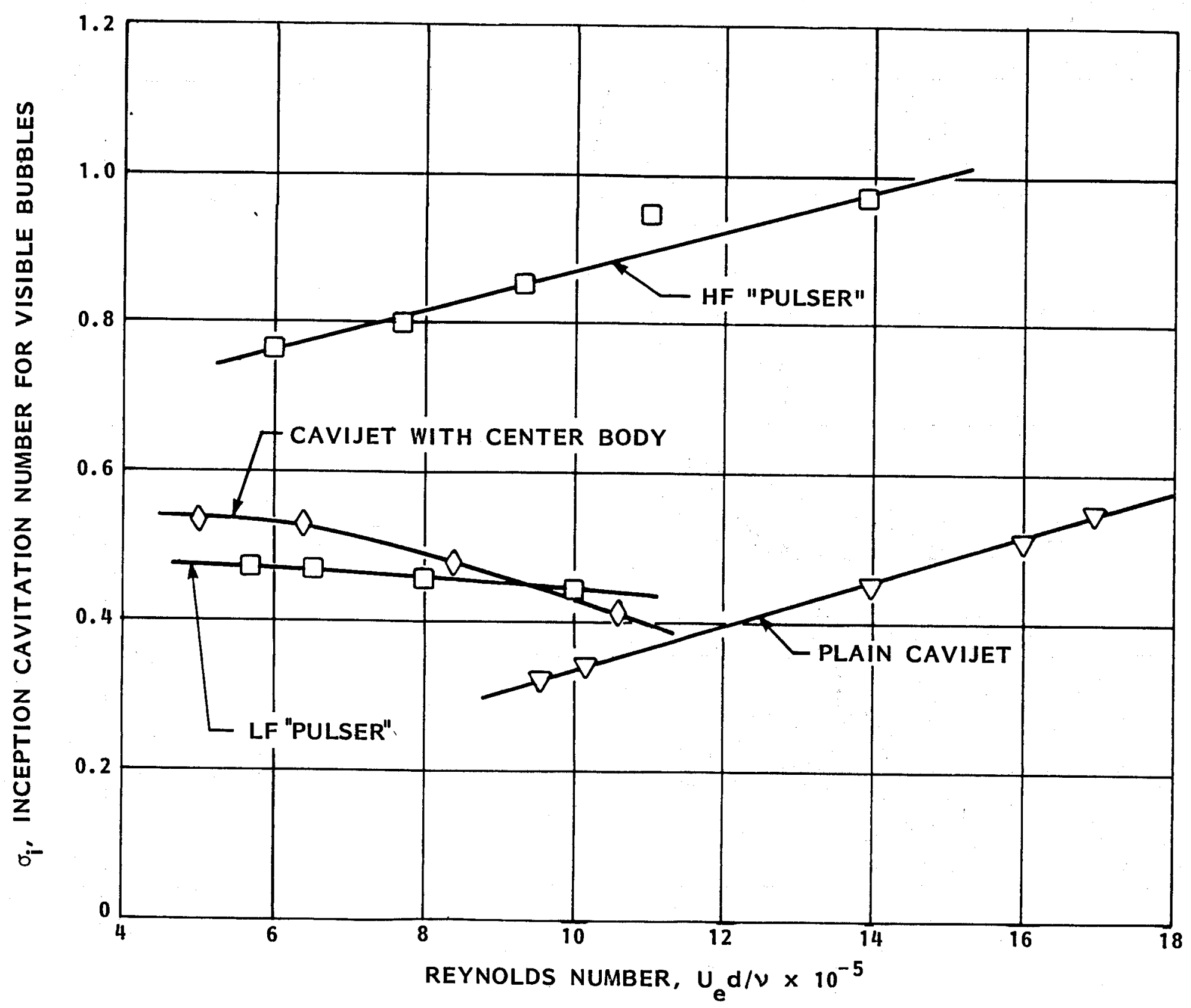

FIGURE 49 - CAVITATION INCEPTION FOR VARIOUS CAVIJETS IN HPC 


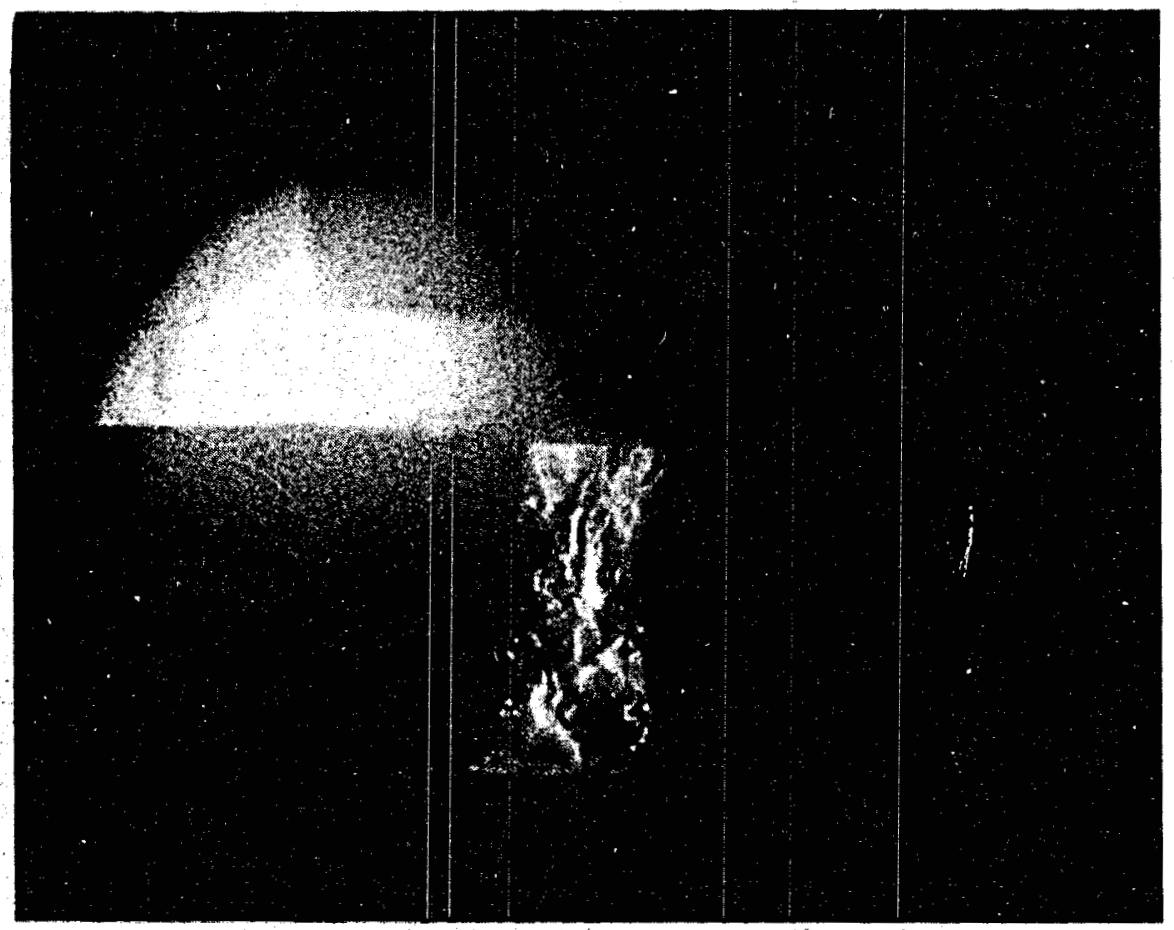

$\Delta p=5.5 \mathrm{MPa}(800 \mathrm{psi}), \sigma=0.25 ; \mathrm{d}_{1}=6.4 \mathrm{~mm}(0.250 \mathrm{in.})$

$d_{2}=6.4 \mathrm{~mm}(0.25 \mathrm{in.}), d_{T}=31.8 \mathrm{~mm}(1.25 \mathrm{in.}), L=11.2 \mathrm{~mm}(0.44 \mathrm{in.})$ $X=22.4 \mathrm{~mm}(0.88 \mathrm{in.})$

\section{FIGURE 50 - CAVITATION PATTERN FOR SELF-EXCITED} RESONANT CAVIJET NOZZLE ("PULSER") 


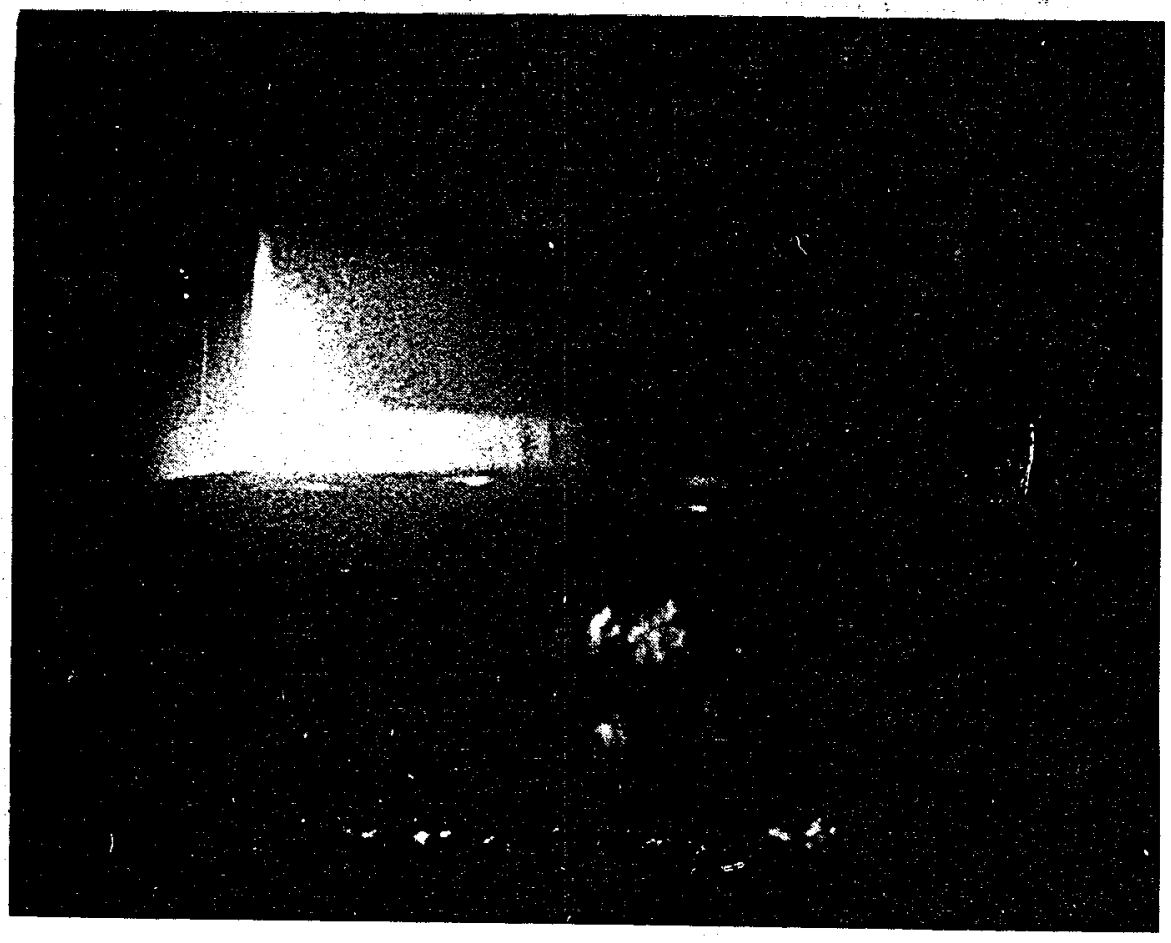

$$
\begin{aligned}
& \Delta p=5.5 \mathrm{MPa}(800 \mathrm{psi}), \sigma=0.25 ; d=7.1 \mathrm{~mm}(0.28 \mathrm{in} .) \\
& d_{c b}=3.6 \mathrm{~mm}(0.14 \mathrm{in} .), X=22.4 \mathrm{~mm}(0.88 \mathrm{in} .)
\end{aligned}
$$

FIGURE 51 - CAVITATION PATTERN FOR CAVIJET WITH CENTERBODY 


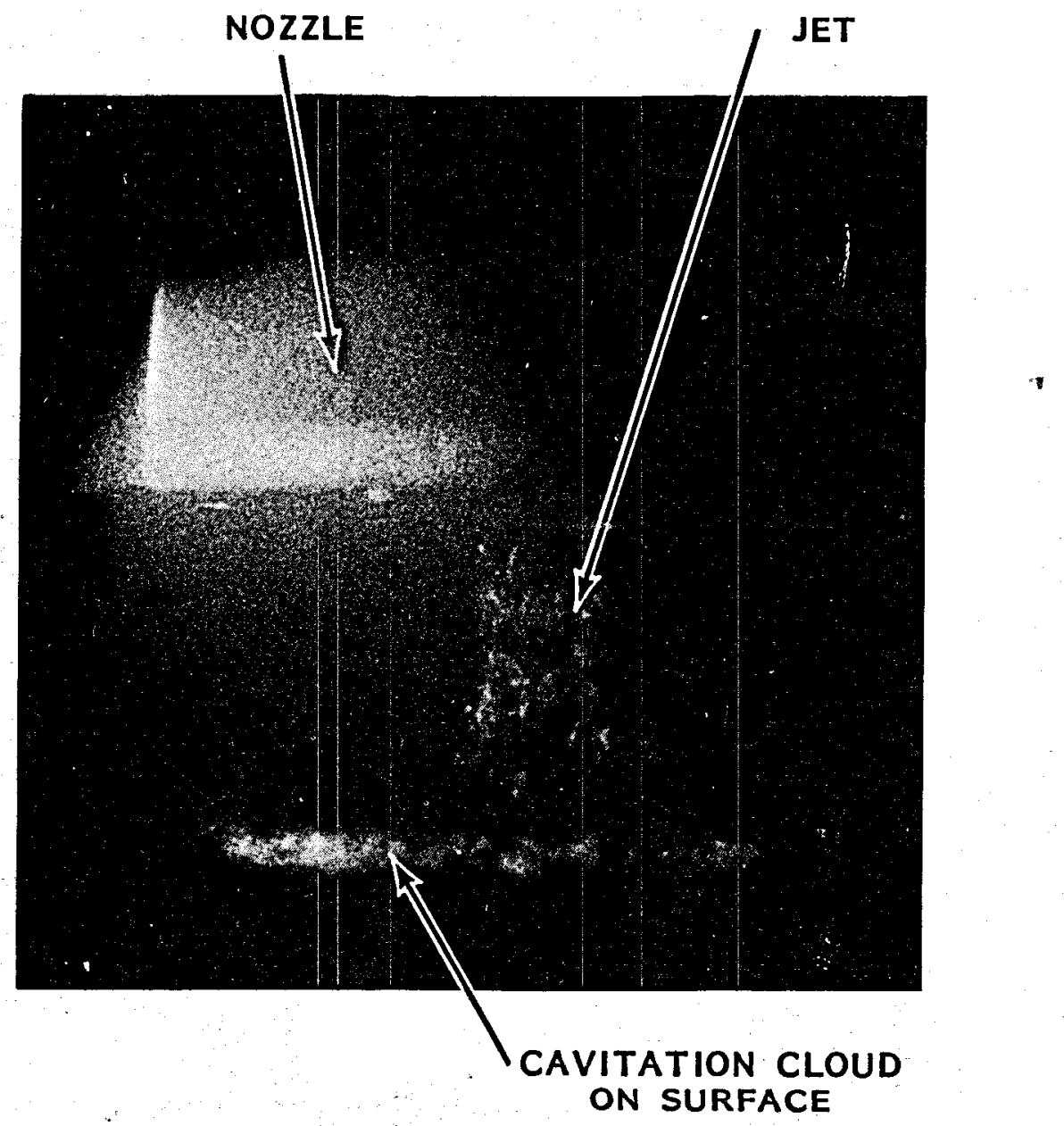

$$
\begin{aligned}
& \Delta p=5.3 \mathrm{MPa}(800 \mathrm{psi}), \sigma=0.25 ; d=6.9 \mathrm{~mm}(0.27 \mathrm{in.}), \\
& X=22.4 \mathrm{~mm}(0.88 \mathrm{in} .)
\end{aligned}
$$

FIGURE 52 - CAVITATION PATTERN FOR A CONVENTIONAL DRILL BIT NOZZLE 
JET DIA. $=2.2 \mathrm{~mm}(0.086 \mathrm{in.})$

$\Delta p=6.9 \mathrm{MPa}(1000 \mathrm{psi})$

BEREA SANDSTONE SPECIMEN NO. 1

AT $\sigma \approx 0.015$

O PLAIN CAVIJET

"PULSER" NOZZLE

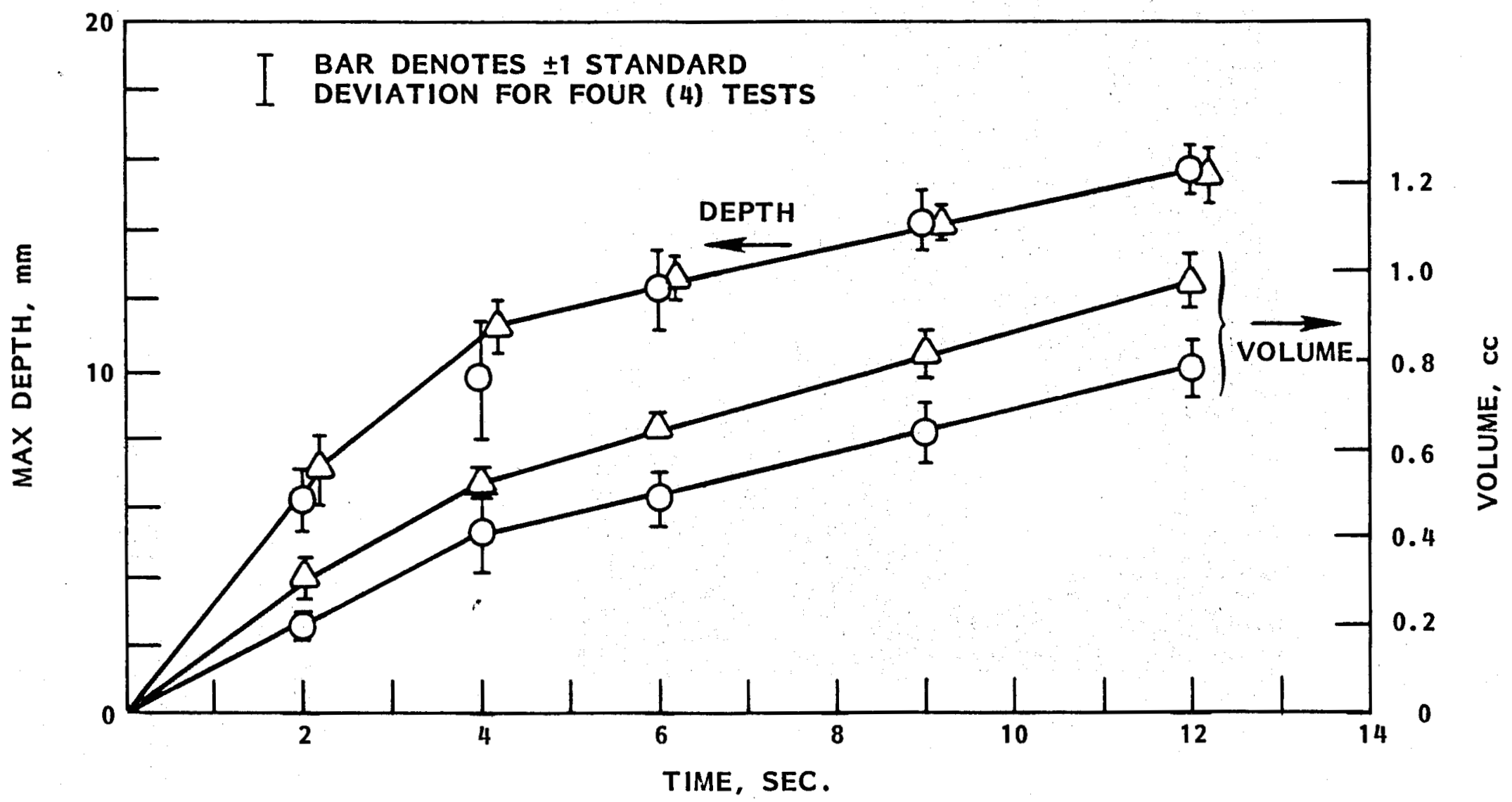

FIGURE 53 - MAXIMUM DEPTH AND VOLUME EROSION HISTORIES FROM TESTS IN THE CAVIJET ATMOSPHERIC PRESSURE FACILITY 
CONCEPTS

"LAID-BACK PULSER"

"PULSER-FED" CAVIJET

OTHER

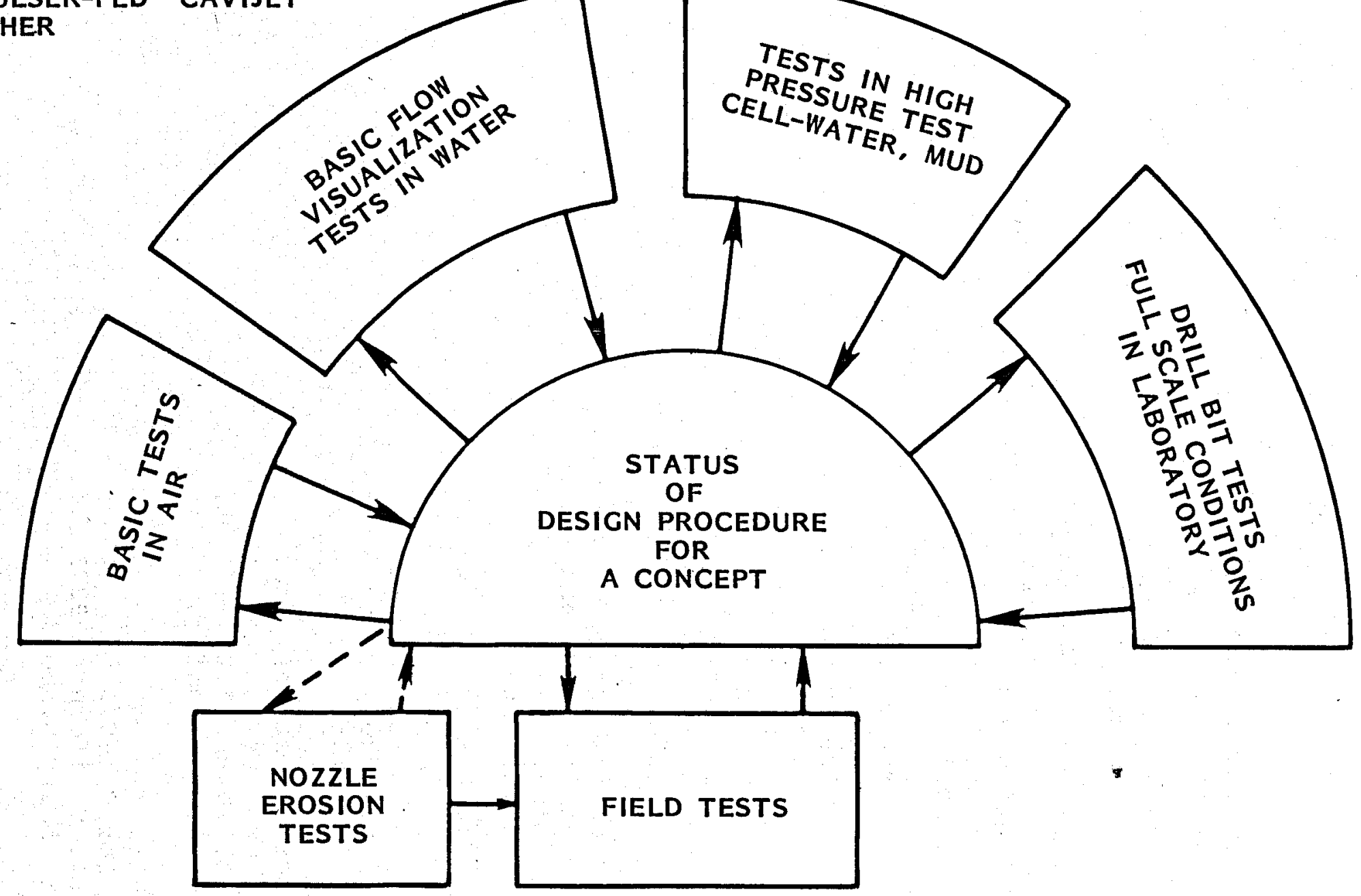


DISTRIBUTION :

TID-4500-R66-UC-66C (675)

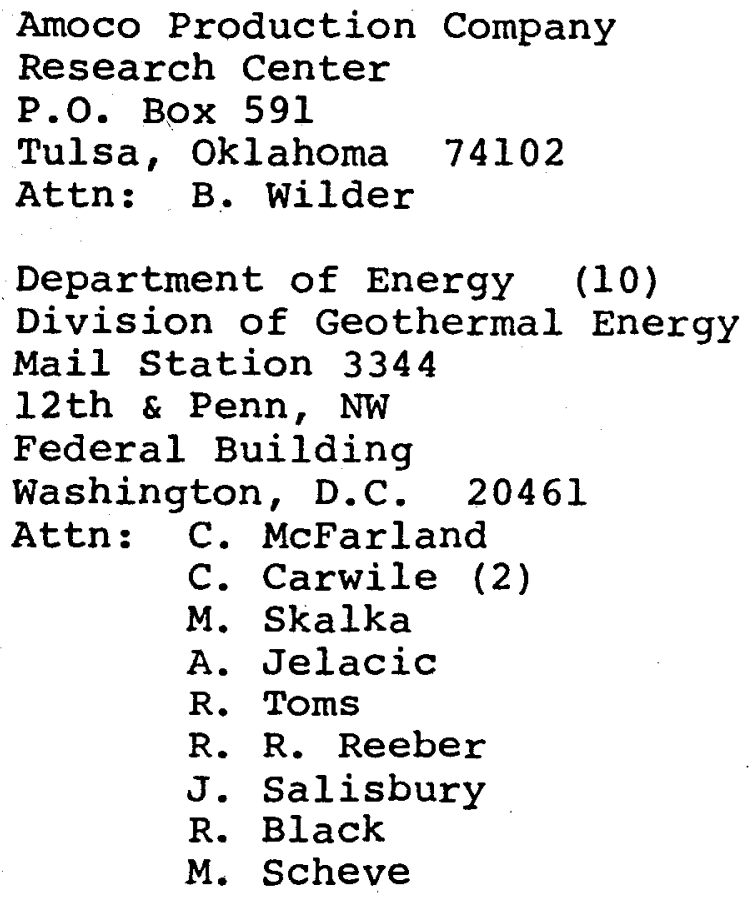

Dresser Industries, Inc. P.O. Box 24647 
DISTRIBUTION (Continued)

Halliburton

Drawer 1413

Duncan, Oklahoma 73533

Attn: Dwight smith

Hydronautics, Incorporated

7210 Pindell School Road

Laurel, MD 20810

Attn: A. F. Conn

V. E. Johnson, Jr.

H. L. Liu

G. S. Frederick

Loffland Brothers Company

P.O. Box 2847

Tulsa, Oklahoma 74101

Attn: H. E. Mallory

Los Alamos National Scientific Laboratory

Mail Stop 570

Los Alamos, New Mexico 87545

Attn: John C. Rowley

Mobil Research and Development Corporation

Field Research Laboratory

P.O. Box 900

Dallas, Texas 75221

Attn: W. Gravley

NL Baroid Petroleum Services

City Centre Building, suite $365 \mathrm{~W}$

6400 Uptown Boulevard

Albuquerque, New Mexico 87110

Attn: Gene Polk

NL Petroleum Services

P.O. Box 1473

Houston, Texas 77001

Attn: J. Fontenot

Otis

P.O. Box 34380

Dallas, Texas 75243

Attn: W. D. Rumbaugh 
DISTRIBUTION (Continued)

Phillips Petroleum Company

P.O. Box 239

Salt Lake City, Utah 84110

Attn: Earl Hoff

Smith Tool Company

P.O. Box C-19511

Irvine, California 92713

Attn: J. Vincent

Texas A\&M University

College Station, Texas 77843

Attn: M. Friedman

Dept. of Geology

Union Geothermal Division

Union Oil Company of California

Union Oil Center

Los Angeles, California 90017

Attn: D. E. Pyle

Shell oil Co.

Two Shell Plaza

P.O. Box 2099

Houston, Texas 77001

Attn: W. E. Bingman

400 C. Winter

1000 G. A. Fowler

1100 C. D. Broyles

1130 H. E. Viney

2000 E. D. Reed

2300 J. C. King

2320 K. Gillespie

2325 R. E. Fox

2328 J. H. Barnette

2500 J. C. Crawford

2513 W. B. Leslie

4000 A. Narath

4200 G. Yonas

4300 R. L. Peurifoy, Jr.

4400 A. W. Snyder

4443 P. Yarrington

4500 E. H. Beckner 
DISTRIBUTION (Continued)

$\begin{array}{ll}4700 & \text { J. H. Scott } \\ 4710 & \text { G. E. Brandvold } \\ 4720 & \text { D. G. Schueler } \\ 4740 & \text { R. K. Traeger } \\ 4741 & \text { J. R. Kelsey } \\ 4741 & \text { C. C. Carson } \\ 4743 & \text { H. C. Hardee } \\ 4750 & \text { V. L. Dugan } \\ 4751 & \text { J. R. Tillerson } \\ 4751 & \text { D. A. Glowka } \\ 4752 & \text { H. M. Dodd } \\ 4754 & \text { A. F. Veneruso } \\ 5000 & \text { J. K. Galt } \\ 5510 & \text { D. B. Hayes } \\ 5512 & \text { D. F. McVey } \\ 5512 & \text { A. Ortega } \\ 5530 & \text { W. Herrmann } \\ 5532 & \text { B. M. Butcher } \\ 5533 & \text { J. M. McGlaun } \\ 5600 & \text { D. B. Shuster } \\ 5620 & \text { M. M. Newsom } \\ 5800 & \text { R. S. Claassen } \\ 5810 & \text { R. G. Kepler } \\ 5812 & \text { C. J. M. Northrup, Jr. } \\ 5812 & \text { B. T. Kenna } \\ 5813 & \text { P. B. Rand } \\ 5830 & \text { M. J. Davis } \\ 5832 & \text { R. W. Rohde } \\ 5832 & \text { R. J. Salzbrenner } \\ 5833 & \text { J. L. Jellison } \\ 5836 & \text { J. L. Ledman } \\ 5840 & \text { N. J. Magnani } \\ 3141 & \text { L. J. Erickson } \\ 3151 & \text { W. L. Garner } \\ 8214 & \text { M. A. Pound } \\ & \end{array}$

